\title{
Transport phenomena, equations and numerical methods
}

Sri Redjeki Pudjaprasetya

October 10, 2021 
Dedicated to the Djohan family;

Warsoma, Yovita, Ratna, Irma, and all my students. 


\section{Contents}

1 Decay problem 11

1.1 Time integration scheme . . . . . . . . . . . 12

2 Harmonic oscillator 17

2.1 Fluid motion in a U-tube . . . . . . . . . . . . . . 17

2.2 Motion of a bouyant object . . . . . . . . . . . . . . 18

2.3 Particle trajectory under Coriolis force . . . . . . . . . . 20

3 Finite Difference for Diffusion Equation 25

3.1 Finite Difference Method . . . . . . . . . . . . . 25

3.2 Diffusion equation for heat conduction . . . . . . . . . . 27

3.3 The Euler explicit method . . . . . . . . . . . . . . . 29

3.3.1 Modification for a Neumann boundary condition . . 34

3.4 Implicit method . . . . . . . . . . . . . . . . 35

3.5 Theta-method and Crank-Nicolson method . . . . . . . . . . 36

3.6 Stefan Problem ................ 37

4 Finite Difference for Convection Equation 45

4.1 Characteristic method ............. 45

4.2 Upwind method . . . . . . . . . . . . 47

4.3 Error in numerical solutions . . . . . . . . . . . 52

5 Conservative Problems \& Finite Volume Method 57

5.1 Riemann problem ............... 58

5.2 Finite volume method .............. 62

5.2.1 Godunov method . . . . . . . . . . . . 65

5.3 The kinematic LWR model for traffic flow . . . . . . . . . . . 68

5.4 Shock and rarefaction waves in traffic flow . . . . . . . . 74

5.5 Finite volume method for the kinematic LWR model . . . . . 75 
6 The shallow water equations $\quad 79$

6.1 Water waves and the shallow water model . . . . . . . . 79

6.2 Direct derivation of the shallow water equations . . . . . . 82

6.3 Linear Shallow Water Equations . . . . . . . . . . . . 87

6.4 Finite difference method . . . . . . . . . . . . . . . . 91

6.5 Riemann invariant form . . . . . . . . . . . . . . . . . 95

7 Staggered grid method for SWE $\quad 99$

7.1 The leapfrog method . . . . . . . . . . . . . . . . 999

7.2 Conservative scheme for the half linear SWE . . . . . . 105

7.3 The full nonlinear SWE . . . . . . . . . . . . . . . 110

7.3.1 Wet-dry procedures . . . . . . . . . . 110

7.3.2 Shapiro filter . . . . . . . . . . . . . . . . 111

7.4 Staggered finite volume method for 2D SWE . . . . . . . 114 


\section{List of Figures}

1.1.1 Computed $c(t)$ together with the exact solution, using $\lambda \Delta t=$ $-0.1,0.5,-1,-2$, and $-4 \ldots \ldots \ldots \ldots \ldots$

1.1.2 The ratios $c^{n+1} / c^{n}$ as a function of $|\lambda| t$ for time integration schemes: Euler explicit, Euler implicit, trapezoidal, and mid point rule, compare with the exact ratio $\exp (\lambda \Delta t) \ldots \ldots \ldots 15$

2.3.1 The parcel trajectory in a rotating frame. (a) Trajectory from the implicit Euler scheme. (b) Trajectory from trapezoidal scheme. Reference location in the fixed frame of reference is indicated with the dot location. . . . . . . . . . .

3.1.1 A connection diagram between the differential equation and its difference equation counterpart. . . . . . . . . . . . 26

3.2.1 A segmen $[x, x+\Delta x]$ in the lateraly insulated bar. . . . . . 28

3.3.1 The computational domain with positions of initial condition, left and right boundary conditions. . . . . . . . . 30

3.3.2 Stencil of the Euler explicit (or FTCS) method for the diffusion equation. . . . . . . . . . . . 30

3.3.3 Plots of numerical solutions at subsequent times showing the decay of temperature because of zero temperatures at both ends, computed using parameters $\kappa=0.3, L=1$, $\Delta x=0.05, \Delta t=0.125 e-2 \ldots \ldots \ldots \ldots \ldots$

3.4.1 Stencil of the BTCS implicit method for the diffusion equation.

3.5.1 Stencil of the theta-method. . . . . . . . . . . . . 36

3.6.1 Description of one-dimensional Stefan problem. . . . . . . 37

3.6.2 Relation between internal energy (enthalpy) $E$ with respect to temperature $T$ and its inverse temperature $T$ as a function of enthalpy $E . \ldots \ldots \ldots \ldots \ldots \ldots \ldots$

3.6.3 Partition of the computational domain $[0, l]$ in $N x$ control volumes. . . . . . . . . . . . . . . . . 40 
3.6.4 (Left) Snapshots of enthalpy curves $E(x, t)$. (Right) Snapshots of temperature curves $T(x, t)$. The interface between liquid and solid can be detected in two ways: from the temperature curve as a position with $T(x, t)=T_{m}$, and from the enthalpy curve as a position of discontinuous $E(x, t) \ldots$. . . 41

3.6.5 Three phase diagram of water $\mathrm{H}_{2} \mathrm{O}$. . . . . . . . . . . 43

3.6.6 The snapshots of temperature and enthalpy curves on the changing phase proses of water. . . . . . . . . . .

4.1.1 Characteristic lines $x(t)=d t+k, k \in \mathbb{R}$ used for constructing the analytical solution. . . . . . . . . . . . 46

4.2.1 Stencil of the upwind method. . . . . . . . . . . . . . . . . 48

4.2.2 Computational domain with locations of initial and boundary conditions, and computed values $u_{j}^{n}, j=2, \cdots, N_{x}+1$, $n=2, \ldots, N t \ldots \ldots \ldots \ldots \ldots \ldots \ldots$

4.2.3 Stability condition $C=\frac{d \Delta t}{\Delta x} \leq 1$ with a geometrical interpretation on the computational mesh. . . . . . . . . 50

4.2.4 Numerical approximation of a sine wave at time $t=1 \mathrm{com}-$ puted using the upwind scheme.

4.3.1 Numerical solution of the Lax-Wendroff scheme together with the analytical solution at $t=1 . \ldots \ldots \ldots$

4.3.2 Comparison of exact solution with the upwind numerical method (left), and the Lax-Wendroff method (right). . . . . . 56

5.1.1 Intersecting characteristics and the shock wave. . . . . . . 59

5.1.2 Separating characteristics and the rarefaction wave. . . . . 59

5.1.3 A shock formed between crossing characteristics. . . . . . 60

5.2.1 The spatial domain with the staggered grid points. . . . . . . 63

5.2.2 Sketch of the spatial domain in the finite volume method, and illustration of the piecewise constant numerical solution at time level $t^{n} \ldots \ldots \ldots$. . . . . . . . . . . . 64

5.2.3 Shock wave solutions at time $t=1$ calculated using Godunov method (left) and Upwind method (right), in comparison with the analytical solution. . . . . . . . . . 66

5.3.1 A pulse of traffic density $n(x, t)($ cars $/ \mathrm{km})$ in the macroscopic approach. . . . . . . . . . . . . . . . . 69

5.3.2 Illustration of the control volume $[x, x+\Delta x]$ with the in-out relation. . . . . . . . . . . . . . .

5.3.3 Observed velocity-density-flux relationships (data source: NaviGAtor). . . . . . . . . . . . . . . . . . 70

5.3.4 The Greenshield velocity $v(n)$ (left), and the corresponding flux $f(n)=n v(n)$ (right). 
5.3.5 Several traffic flow models (left) velocity density relationship, (right) flux density relationship. . . . . . . . . . 72

5.4.1 (Top) The flux curve with two traffic situations $A$ and $B$, connected with a segment line with gradient $m_{A B}$. (Bottom) Top view of a dense traffic condition $B$ propagating backward to the less dense traffic condition $A$. . . . . . . . . 75

5.4.2 Typical profile of traffic density at subsequent times when traffic light turns green. . . . . . . . . . . . 76

5.5.1 Upwind approximation for $F_{i+\frac{1}{2}}$, holds for every time iteration. . . . . . . . . . . . . . . . 77

6.1.1 Sketch of fluid domain. . . . . . . . . . . . . . . . . 80

6.2.1 Wave classification based on depth and wavelength. . . . . . 83

6.2.2 A control volume in the derivation of SWE. . . . . . . . . . 84

6.3.1 Left and right running characteristic of the linear SWE (6.3.6, $6.3 .7 \ldots \ldots \ldots \ldots \ldots$. . . . . . . . . . . . 88

6.3.2 Sketch of an inclined channel and notations. . . . . . . . 90

6.5.1 Left running characteristics with gradient $\frac{d x}{d t}=-c_{0}$ of (6.5.4) and right running characteristics with gradient $\frac{d x}{d t}=c_{0}$ of (6.5.3). . . . . . . . . . . . . . . 96

7.1.1 (Top left and right) Stencils of the original leapfrog scheme and its equivalent. (Bottom) Illustration of staggered grid, with a cell for mass and a cell for momentum. . . . . . . . 100

7.1.2 (Left) The discontinuity remains for a long period of time. (Right) The discontinuity decays after some time. . . . . . 105

7.2.1 A sketch of fluid layer and notations. . . . . . . . . . . 106

7.2.2 Staggered grid with configuration of calculated variables $h$ and $u$. . . . . . . . . . . . . . . . . . . . . . . 106

7.2.3 Transmitted and reflected waves produce by a wave propagates over a bottom step-downwards (left) and a bottom step-upwards (right). . . . . . . . . . . . . . . . . . . 109

7.2.4 Shoaling effect on waves propagating over a decreasing depth.109

7.3.1 In a computation that involves dry areas the still water level is modified to $\eta(x, 0)=\max (0,-d(x)) \ldots \ldots \ldots 111$

7.3.2 Plot of surface wave uprush on a sloping beach at subsequent times. . . . . . . . . . . . . . . . . . 112

7.3.3 Surface profiles in dam break simulations; (left) the wet-bed case, (right) the dry-bed case.

7.4.1 Arakawa-C grid with cells for mass, momentum $-x$, and momentum-y. Hard-wall boundaries are applied along the four sides. 
7.4.2 Circular waves resulting from a point-source disturbance. . 116

7.4.3 (Left) the obstacle, (right) waves interact with an obstacle. . 117 


\section{Preface}

This book offers a preliminary overview of transport phenomena. Topics covered are the basic introduction on several aspects of heat transfer, mass transfer, and fluid mechanics, focussing on issues arising from conservation laws. Conservation laws arise when some (physical) quantity is conserved, for example, mass or momentum. Thus they are frequently encountered in a wide range of scientific and engineering fields.

Partial differential equations arising from conservation laws are mostly non-linear, often they also admit discontinuous shock wave solutions. In applications, shock wave solutions may be found as bores in rivers, sonic boom, or traffic jam. This book contains a comprehensive coverage of numerical techniques and analysis, as well as theoretical development, which is vital to the understanding and implementation of applications.

The simple finite difference are discussed for solving linear ordinary differential equations and partial differential equations. Our discussion includes basic terminologies such as stability, consistence, and convergence. Also includes ways to implement boundary conditions (Dirichlet or Neumann) numerically. These kind of discussions are often skipped in numerical books, which is very unfortunate because misapplying boundary conditions will lead to completely different or wrong solutions.

Furthermore, conservative models are generally non-linear and sometimes they have discontinuous solutions. All of these give additional challenges for the numerical solutions, especially since they are vulnerable to instability. Therefore, here we present a robust class of numerical methods, namely the finite volume method. This method is discussed to solve both linear and nonlinear conservation equations.

This book provides both theoretical and numerical aspects of the problem, as well as the modeling and interpretations. The approach used in here is constructive rather than theoretical. We hope this will help students to 
develop an understanding of both the mathematical and computational issues that arise as they learn to deal with problems involving differential equations.

All the numerical methods presented here has been implemented using Matlab, and students are encouraged to try to compose their own codes. It is very important to work on the exercises to develop skills on the analytical as well as computational aspects of the subject. To facilitate learning, some basic source codes in Matlab are available on the website https://srpudjap.wordpress.com These codes can be used as a guide for the development of programs in other computer languages. Accompanying material such as animations, and further discussion on particular problems or projects are also available on the same website. We hope that this may provide an excellent learning environment for understanding the subject.

This book is designed for researchers and students studying mathematics, engineering, and sciences. The prerequisites are a solid understanding of calculus and a basic understanding of differential equations. Readers with a solid foundation in ordinary and partial differential equations will benefit greatly from this book. The original text was based on author's lecture notes for senior-level undergraduate and beginning graduate students at Mathematics Department, Institut Teknologi Bandung. In recent years, the author had used Chapters 1 through 6 in a 4-semester-hour course on Numerical Methods for Conservative Problems for the final year of undergraduate Mathematics students. Another composition of Chapters $1,2,4,6,7$ has been used in a course on Computational Fluid Dynamics for magister students in science. Some parts of the material, such as Subsections 2.3, 3.6 and 5.3-5.5, have been used in several workshops or short courses.

\section{Acknowledgement}

I would like to thank Institut Teknologi Bandung and ITB Press, for giving me the opportunity to publish this book. Special thanks to the students who took my courses in Computational Fluid Dynamics and Numerical Methods for Conservative Problems from year 2016 to 2021. They have all given me a lot of encouragement to write this book and have allowed me to improve its presentation. Thanks to my daughter Yovita Adj for conducting proofreading the first draft. Of course, there may be errors and typos in this first volume; therefore, corrections, comments, and suggestions for improvement are greatly appreciated. 


\section{Chapter 1}

\section{Decay problem}

On this first chapter we study a problem of pollutant concentration that has a simple mathematical model. Through this problem, various numerical schemes of an ordinary differential equation will be discussed, as well as important requirements for a numerical scheme as an approximation of the original differential equation. Upon presenting the problem, the modeling aspect is discussed.

A pollutant mixture with a concentration of $c_{i n}$ flows into the lake with influx of $Q_{i n}$. Then it is discharged with outflux of $Q_{o u t}$. Pollutants in the lake are assumed to be immediately mixed, and $Q_{i n}$ and $Q_{o u t}$ can be functions of time $t$ in general. Let $c(t)$ denote pollutant concentration in the lake at time $t, c(0)$ the initial concentration, and $V(t)$ the lake volume at time $t$. Dimensions for variables $c, Q, V$ are $\left[M / L^{3}\right],\left[L^{3} / T\right],\left[L^{3}\right]$, respectively, and we use MCS units here. The volume balance dictates that the rate of change of lake volume equals to $Q_{i n}-Q_{o u t}$, explicitly

$$
\frac{d V}{d t}=Q_{\text {in }}-Q_{o u t} .
$$

Conservation of mass states that the rate of change of pollutants in the lake equals the difference between the influx and outflux of pollutants:

$$
\frac{d(c V)}{d t}=Q_{i n} c_{\text {in }}-Q_{o u t} c .
$$

Two equations above can be combine into one equation below:

$$
\frac{d c}{d t}=-\frac{Q_{i n}}{V}\left(c-c_{i n}\right) .
$$

Please check! When the value of $c_{i n}=0$, Equation (1.0.1) is simplified to:

$$
\frac{d c}{d t}=\lambda c, \quad \text { with } \quad \lambda=-Q_{\text {in }} / V .
$$


According to the equation (1.0.2), the concentration $c(t)$ depends only on the ratio $Q_{i n} / V$. If we further assume $Q_{i n}$ and $V$ are constant, equations (1.0.2) has an explicit solution, and that is

$$
c(t)=c_{0} e^{\lambda t} .
$$

The parameter $1 / \lambda=-V / Q_{i n}$ determines the time required to empty the lake of volume $V$ with outflux $Q_{i n}$; this parameter will determine the characteristic time scale of the system, also known as the flushing time, relaxation time, or age. Consider the case when $Q_{i n}=Q_{o u t}$, that produces a constant $V$, Flushing time is the time needed to replace fluid in a lake with a certain volume $V$ by pumping at a discharge rate $Q$. Mathematically speaking, this parameter denotes the time at which the concentration has reduced by a factor $1 / e$. Through this simple ordinary differential equation, we will review several numerical integration method, including its accuracy, stability, and convergence.

\subsection{Time integration scheme}

The following are standard finite difference formulas often used to approximate the differential equation (1.0.2).

$$
\begin{array}{llr}
\text { Euler's explicit } & \frac{c^{n+1}-c^{n}}{\Delta t}=\lambda c^{n} & \tau_{\Delta t}=c_{t t}^{n} \frac{\Delta t}{2}+\mathcal{O}\left(\Delta t^{2}\right) \\
\text { Euler's implicit } & \frac{c^{n+1}-c^{n}}{\Delta t}=\lambda c^{n+1} & \tau_{\Delta t}=-c_{t t}^{n} \frac{\Delta t}{2}+\mathcal{O}\left(\Delta t^{2}\right) \\
\text { Trapezoidal rule } & \frac{c^{n+1}-c^{n}}{\Delta t}=\frac{\lambda}{2}\left(c^{n+1}+c^{n}\right) & \tau_{\Delta t}=-c_{t t t}^{n} \frac{\Delta t^{2}}{12}+\mathcal{O}\left(\Delta t^{3}\right) \\
\theta \text {-method } & \frac{c^{n+1}-c^{n}}{\Delta t}=\lambda\left(\theta c^{n+1}+(1-\theta) c^{n}\right) & \\
\text { Midpoint rule } & \frac{c^{n+1}-c^{n-1}}{2 \Delta t}=\lambda c^{n} & \tau_{\Delta t}=c_{t t t}^{n} \frac{\Delta t^{2}}{6}+\mathcal{O}\left(\Delta t^{3}\right)
\end{array}
$$

The error $\tau_{\Delta t}$ denotes the difference between each differential equation with the approximate difference equations. The accuracy order of difference equations are determined from the order of $\tau_{\Delta t}$.

Task: Derive all the error above. Hint: for deriving the error term of the trapezoidal rule, you may need to implement $c_{t}=\lambda c$

It is easy to check that the Euler explicit formula is equivalent with the recursion formula $c^{n+1} / c^{n}=r_{\text {exp }}$, with

$$
r_{\exp }=(1+\lambda \Delta t) .
$$


Similarly, the other formulas can be written as recursive formulas with ratio

$$
\begin{aligned}
r_{\text {imp }} & =1 /(1-\lambda \Delta t), \\
r_{\text {trap }} & =\frac{1+\frac{1}{2} \lambda \Delta t}{1-\frac{1}{2} \lambda \Delta t} .
\end{aligned}
$$

Task: Discussion about spurious solutions of the midpoint-rule. As a second order difference equation the midpoint-rule owes a spurious solution which need to be avoided. Further discussion about this, see [17] pages 24-25.

\section{Exercise 1.1.1.}

(Time integration scheme)

Consider $c_{t}=\lambda c$, with $c(0)=1$. Use $\lambda \Delta t=-0.1$ to calculate $c(t)$, for 20 time steps using the above numerical integrations: Euler's explicit, implicit, and trapezoidal. Reproduce Figure 1.1.1 (left) that shows the numerical results together with the exact solution $c_{\text {exact }}(t)=e^{\lambda t}$. It is shown that the numerical solutions from the explicit, implicit and trapezoidal schemes are all nicely approximate the analytical solution. As a second order scheme, the trapezoidal approximates better compare with the other two schemes. But if we repeat the same calculations using different values of $\lambda \Delta t$, strange behavior arise. Figure 1.1.1 (right) displays several computation results using $\lambda \Delta t=-0.5,-1,-2$, and -4 . Clearly, the computed results behaves different qualitatively, and they depends on the choice of $\lambda \Delta t$. A numerical scheme is called stable if and only if the computed solution is finite at all times. In this problem, numerical solutions $c^{n}$, with $n=0,1,2, \cdots$ compose a geometric series with ratios given as (1.1.1-1.1.3). Stability of the scheme directly comes from stability of the geometric series. For consistency and convergence aspects, readers may consult the book [15]. Properties (including stability) of the time difference schemes can directly be obtained from the ratio $c^{n+1} / c^{n}$ curves in Figure (1.1.2).

1. For each figure in 1.1.1 (right), determine which scheme is stable.

2. Use Figure (1.1.2) to explain the behavior of the calculated solutions in Figure 1.1.1.

3. Derive the stability conditions for the three schemes: Euler explicit, Euler implicit, and trapezoidal schemes. Please note that $\lambda$ is negative. 

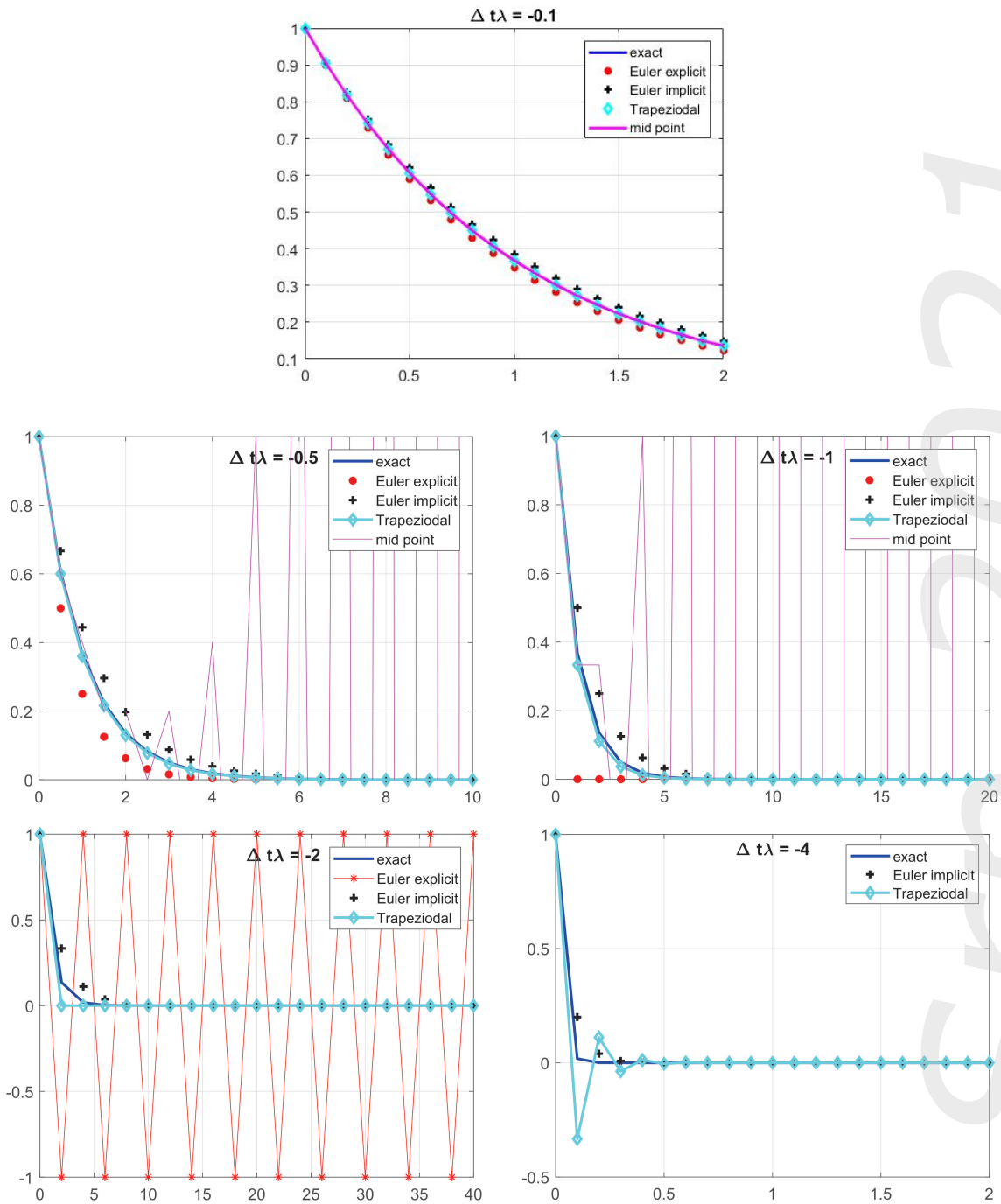

Figure 1.1.1: Computed $c(t)$ together with the exact solution, using $\lambda \Delta t=$ $-0.1,0.5,-1,-2$, and -4 . 


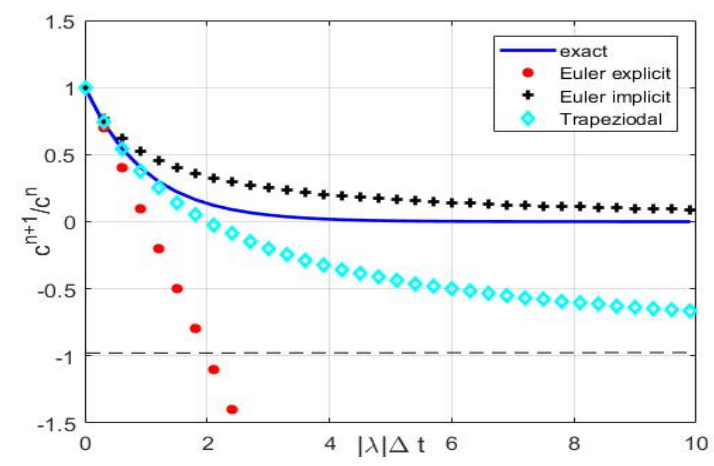

Figure 1.1.2: The ratios $c^{n+1} / c^{n}$ as a function of $|\lambda| t$ for time integration schemes: Euler explicit, Euler implicit, trapezoidal, and mid point rule, compare with the exact ratio $\exp (\lambda \Delta t)$.

\section{Exercise 1.1.2.}

(Pollutant problem)

Suppose $Q_{o u t}=Q_{\text {in }}=Q$, and the volume of the mixture in the lake is $V$, a constant. Mass conservation principle will result in:

$$
V \frac{d c}{d t}=Q\left(c_{i n}-c\right) .
$$

Suppose the concentration of inflow pollutant follows a harmonic function $c_{i n}=\hat{C}(\sin \omega t+1)$. Next, to reduce the number of parameters, we introduce the following non-dimensionless scaled variables:

$$
c^{\prime}=\frac{c}{\hat{C}^{\prime}}, \quad t^{\prime}=\omega t, \quad \Omega=\frac{\omega V}{Q} .
$$

Variables with ' are non-dimensionless. The mass conservation becomes

$$
\frac{d c^{\prime}}{d t^{\prime}}=\frac{1}{\Omega}\left(\sin t^{\prime}+1-c^{\prime}\right)
$$

with initial concentration $c^{\prime}(0)$. We note that the equation in non-dimensionless variables only depends on one parameter only, which is $\Omega$. Parameter $\Omega$ is called buffer, and its interpretation will be clear when we compare $c_{\text {in }}(t)$ with $c(t)$, see exercise 4 .

1. Find the exact solution of (1.1.4). Determine the initial concentration $c^{\prime}(0)$ such that the solution does not contain homogeneous solution. 
2. Implement explicit, implicit, and trapezoidal schemes to find approximate solution of (1.1.4).

3. Discuss stability, consistency, and convergence of all schemes.

4. Calculate the concentration $c^{\prime}(t)$ using initial concentration $c^{\prime}(0)$ as obtained in a. Plot the numerical result against the analytical solution, and plot them together with the inflow $c_{i n}(t)$. Compute for medium buffer $\Omega=2$, with $\Delta t^{\prime}=\frac{\pi}{4}, \frac{\pi}{8}, \frac{\pi}{16}$, large buffer $\Omega=20$, with $\Delta t^{\prime}=\frac{\pi}{8}$, and small buffer $\Omega=0.2$, with $\Delta t^{\prime}=\frac{\pi}{8}$. Interpret the result. Is stability condition of the schemes depends on $\Omega$ ? Give a reason. 


\section{Chapter 2}

\section{Harmonic oscillator}

The harmonic oscillator is a linearized model for many natural phenomena. In this chapter, we discuss three different physical systems (a U-tube, a bouyant object, and the Coriolis force effect) that are governed by a second order differential equation with harmonic-type solutions. Here, we discuss suitable numerical schemes for a harmonic oscillator. A numerical scheme with amplitude preserving properties is required to prevent our simulation from experiencing false damping.

Without damping, the equation for harmonic oscillator is

$$
u^{\prime \prime}+\lambda^{2} u=0 .
$$

The analytical solution is a simple harmonic motion

$$
u(t)=A \cos \lambda(t-\phi),
$$

which is an oscillation around an equilibrium point $u=0$, with a constant amplitude $A$ and frequency $\lambda$. In this section, we discuss finite difference methods for solving the simple harmonic oscillator. It is important that our numerical scheme is free from damping error.

\subsection{Fluid motion in a U-tube}

A U-tube is filled with fluid of density $\rho$. The tube has uniform crosssectional area $A$, and the total length of the fluid in the tube is $L$. Fluid in the tube is put in motion by initially push the liquid column on one side by a distance $\zeta_{0}$, raising the other side by the same distance, see figure below. Neglecting any resistance forces, equations of motion are 


$$
\left(\begin{array}{l}
\zeta_{t} \\
u_{t}
\end{array}\right)=\left(\begin{array}{ll}
0 & 1 \\
-\frac{2 g}{L} & 0
\end{array}\right)\left(\begin{array}{l}
\zeta \\
u
\end{array}\right)
$$

We propose the first order scheme, which we call the zig-zag method, which is a combination of forward Euler for the first equation, and backward Euler for the second equation, to be explicit

$$
\begin{aligned}
& \zeta^{n+1}=\zeta^{n}+\Delta t u^{n} \\
& u^{n+1}=u^{n}-\frac{2 g \Delta t}{L} \zeta^{n+1}
\end{aligned}
$$

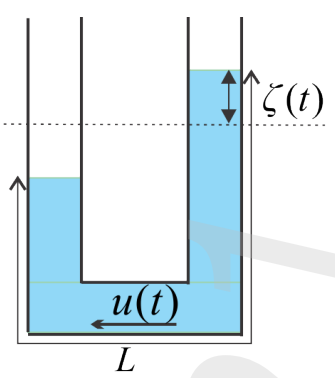

The second approximation we proposed is the trapezoidal method. The following is the second order trapezoidal method

$$
\frac{1}{\Delta t}\left(\begin{array}{l}
\zeta^{n+1}-\zeta^{n} \\
u^{n+1}-u^{n}
\end{array}\right)=\left(\begin{array}{ll}
0 & 1 \\
-\frac{2 g}{L} & 0
\end{array}\right) \frac{1}{2}\left\{\left(\begin{array}{l}
\zeta^{n} \\
u^{n}
\end{array}\right)+\left(\begin{array}{l}
\zeta^{n+1} \\
u^{n+1}
\end{array}\right)\right\} .
$$

\section{Exercise 2.1.1.}

1. The equation of motion (2.1.1) is derived using the conservation of total energy. Total energy $E=$ kinetic + potential, where kinetic energy $K=\frac{1}{2} \rho A L u^{2}$, and potential energy $P=(\rho A \zeta) g \zeta$.

2. What is the frequency $\omega_{0}$ of the simple harmonic motion?

3. Show that zig-zag scheme is of order one, and trapezoidal scheme is of order two.

4. Formulate a stability condition for the zig-zag scheme and trapezoidal scheme.

5. Take parameter $\frac{2 g}{L}=4$, and initial condition $\zeta(0)=0.5, u(0)=0$, simulate the back and forth motion of the water surface. Compare the result with the exact solution.

6. Calculate the total energy $E(t)=\frac{1}{2} \rho A L u^{2}+\rho A g \zeta^{2}$ during the motion. Which scheme gives better result? Explain your answer.

\subsection{Motion of a bouyant object}

Density of seawater is not constant, rather it depends on temperature, salinity, and pressure. Near the surface, water is relatively warm, and 
hence its density is relatively low. In the deeper area, seawater density increases. It is often sufficient to approximate seawater density with a linear function as

$$
\rho_{a m b}(z)=\rho_{0}\left(1+\frac{N^{2}}{g}|z|\right),
$$

where $\rho_{0}$ is density at the surface, and $z$ is the vertical axis. The approximation (2.2.1) is known as the Burnt-Väisälä model, whereas $N$ the Burnt-Väisälä frequency. An object with mass $M_{o b j}$, volume $V_{o b j}$ is released in a water column. Instead of full gravity force, it will experience a reduced gravity force by a bouyancy force, see figure below. According to Archimedes' principle, the resultant force is the difference between the object's mass with the mass of fluid replaced by the object's volume. When we neglect all frictions, the Newton second law for vertical acceleration read as

$$
M_{o b j} \frac{d w}{d t}=-g\left(M_{o b j}-M_{a m b}\right),
$$

or equivalently

$$
\frac{d w}{d t}=-g \frac{\rho_{o b j}-\rho_{a m b}}{\rho_{o b j}} .
$$

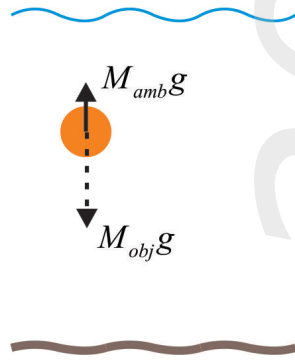

The bouyancy force on the right-hand side of the latter equation varies in magnitude and sign in dependence of the object's location $z$. On the other hand, the location of the object $z$ changes owing to its vertical speed $w$ according to:

$$
\frac{d z}{d t}=w .
$$

Equations $(2.2 .2,2.2 .3)$ are coupled to each other. The proposed finite difference scheme for $(2.2 .2,2.2 .3)$ simply the analogue zig-zag scheme from the previous section, as shown below

$$
\begin{aligned}
& \frac{w^{n+1}-w^{n}}{\Delta t}=-g\left(\rho_{o b j}-\rho_{a m b}\left(z^{n}\right)\right) / \rho_{o b j}, \\
& \frac{z^{n+1}-z^{n}}{\Delta t}=w^{n+1} .
\end{aligned}
$$

Argue that the above scheme is an explicit scheme. 


\section{Exercise 2.2.1.}

Assume the density in a $100 \mathrm{~m}$ deep water column increases linearly with depth. The surface density is $1025 \mathrm{~kg} / \mathrm{m}^{3}$ and $N^{2}=10^{-4} \mathrm{~s}^{-2}$, so that the density increases to $1026 \mathrm{~kg} / \mathrm{m}^{3}$ at the bottom. An object with a density of $1025.5 \mathrm{~kg} / \mathrm{m}^{3}$ is released at a depth of say, $80 \mathrm{~m}$.

1. Find the analytical solution which represents the oscillation motion of the bouyant object in a stratified water column. Hint: Eliminate $w$ from $(2.2 .2,2.2 .3)$, and formulate an orde- 2 ordinary differential equation in terms of $z$. Your analytical formulas will be simpler if you re-write the resulting 2 nd order ode in terms of $z^{*}$, which is the deviation of object location with respect to equilibrium position $\bar{z}$. The equilibrium position is attained when the gravity forces balances the bouyancy force.

2. Find the accuracy order of $(2.2 .4,2.2 .5)$ including its stability condition.

3. Implement the finite difference scheme $(2.2 .4,2.2 .5)$ and simulate the oscillation motion of the object. Make sure that your numerical oscillation has a constant amplitude over a long period of computational time.

4. Introduce friction in the model by adding a term $-R|w| w$ on the right hand side of (2.2.2). Discretize this damping term using a formula $-R\left|w^{n}\right| w^{n+1}$.

\subsection{Particle trajectory under Coriolis force}

We all live on a rotating earth. Motions of wind and currents we observe in daily life are motions on a rotating earth. Governing equations of geophysical fluid motion are naturally written with respect to a rotating frame of reference, the earth rotation. Formulation in this rotating frame of reference results in an addition term, i.e. a fictitious Coriolis force. Due to Coriolis effect, wind moves spiral to the right (clockwise) in the Northern Hemisphere, and spiral to the left (anti-clockwise) in the Southern Hemisphere, and in the equator area there is no Coriolis effect, as illustrated in the next figure.

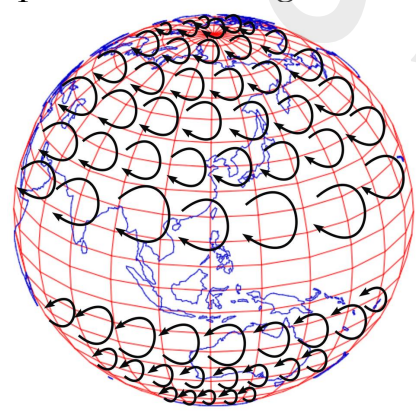

In the discussion follows, we consider a relatively small area on the earth surface, such that we can use Cartesian coordinate system for the position 
of particles. Let $\vec{r}(t)=(x(t), y(t))$ denotes the position of a particle on a rotating earth, and $\frac{\partial \vec{r}}{\partial t}=(u(t), v(t))$ denotes its velocity. In a rotating frame of reference, when other forces are absent except Coriolis, movement of the particle is governed by

$$
\begin{aligned}
& \partial_{t} u-f v=0, \\
& \partial_{t} v+f u=0,
\end{aligned}
$$

with $f=2 \Omega_{r} \sin \phi$ is the Coriolis parameter, $\Omega_{r}=2 \pi / T$ the rotation rate, $T$ the rotation period, and $\phi$ the geographical latitude. The particle trajectory $(x(t), y(t))$ can then be calculated from

$$
\frac{d x}{d t}=u, \quad \frac{d y}{d t}=v .
$$

\section{Exercise 2.3.1.}

1. A particle at an initial position of $x=0 \mathrm{~m}$ and $y=5 \mathrm{~m}$ is obstructed by an initial speed $u=0.5 \mathrm{~m} / \mathrm{s}$ and $v=0.5 \mathrm{~m} / \mathrm{s}$. Under a rotating frame of reference at rate of $f$, show that particle trajectory is a circle with radius $\frac{0.5}{f}$, given by

$$
x(t)=\frac{0.5}{f}(1+\sin f t-\cos f t), \quad y(t)=5+\frac{0.5}{f}(-1+\sin f t+\cos f t) .
$$

This analytical trajectory is a circle, therefore numerical trajectory should also a circle. However, this cannot be achieved using first order schemes, instead it needs a second order scheme. Implementing the second order $\theta$-scheme for $(2.3 .1,2.3 .2)$ will give us

$$
\begin{aligned}
& u^{n+1}=u^{n}+\Delta t f\left((1-\theta) v^{n}+\theta v^{n+1}\right), \\
& v^{n+1}=v^{n}-\Delta t f\left((1-\theta) u^{n}+\theta u^{n+1}\right),
\end{aligned}
$$

with superscript $n=0,1, \cdots$ representing time index. When $\theta=0$ the scheme (2.3.5) is explicit, if $\theta=1$ the scheme is implicit, and $\theta=1 / 2$ the scheme is known as trapezoidal or Crank-Nicolson scheme. Further, particle trajectory $\left(x\left(t_{n}\right), y\left(t_{n}\right)\right)$ as a function of time can be calculated by applying the forward Euler method to (2.3.3). 
2. Conduct the eigenvalues analysis of the amplification matrix $A$ corresponds to (2.3.5) and conclude that the explicit scheme is unconditionally unstable, whereas implicit and trapezoidal schemes are unconditionally stable. Moreover, show that matrix $A$ from the trapezoidal scheme has eigenvalues with spectral radius one, i.e. $\left|\lambda_{1,2}\right|=$ 1 . Hence, this scheme is free from numerical damping error.

3. Implement the implicit and trapezoidal schemes to simulate the particle trajectory. In order to speed up the computational time, just take a dummy frequency $f=-2 * p i / 100 \mathrm{sec}^{-1}$. (Note that Coriolis parameter corresponds to clockwise rotation with a period of 24 hours is $f=2 * \pi / 3600 \mathrm{sec}^{-1}=-7.27 \times 10^{-5} \mathrm{sec}^{-1}$.) Result from the implicit scheme are given in Figure 2.3.1(a). Even tough the implicit scheme is stable, we observe a trajectory spiralling inward and a gradual decrease in speed, which is incorrect. However, the trapezoidal results as in Figure 2.3.1(b) shows the correct results, in which particle's trajectory forms a perfect circle!

Hint: Explicit formulas for the trapezoidal scheme, equivalent to (2.3.5) with $\theta=1 / 2$ is as follows

$$
\begin{aligned}
& u^{n+1}=\left(\left(1-0.25 \alpha^{2}\right) u^{n}+\alpha v^{n}\right) /\left(1+0.25 \alpha^{2}\right), \\
& v^{n+1}=\left(\left(1-0.25 \alpha^{2}\right) v^{n}-\alpha u^{n}\right) /\left(1+0.25 \alpha^{2}\right),
\end{aligned}
$$

with $\alpha=f \Delta t$. 

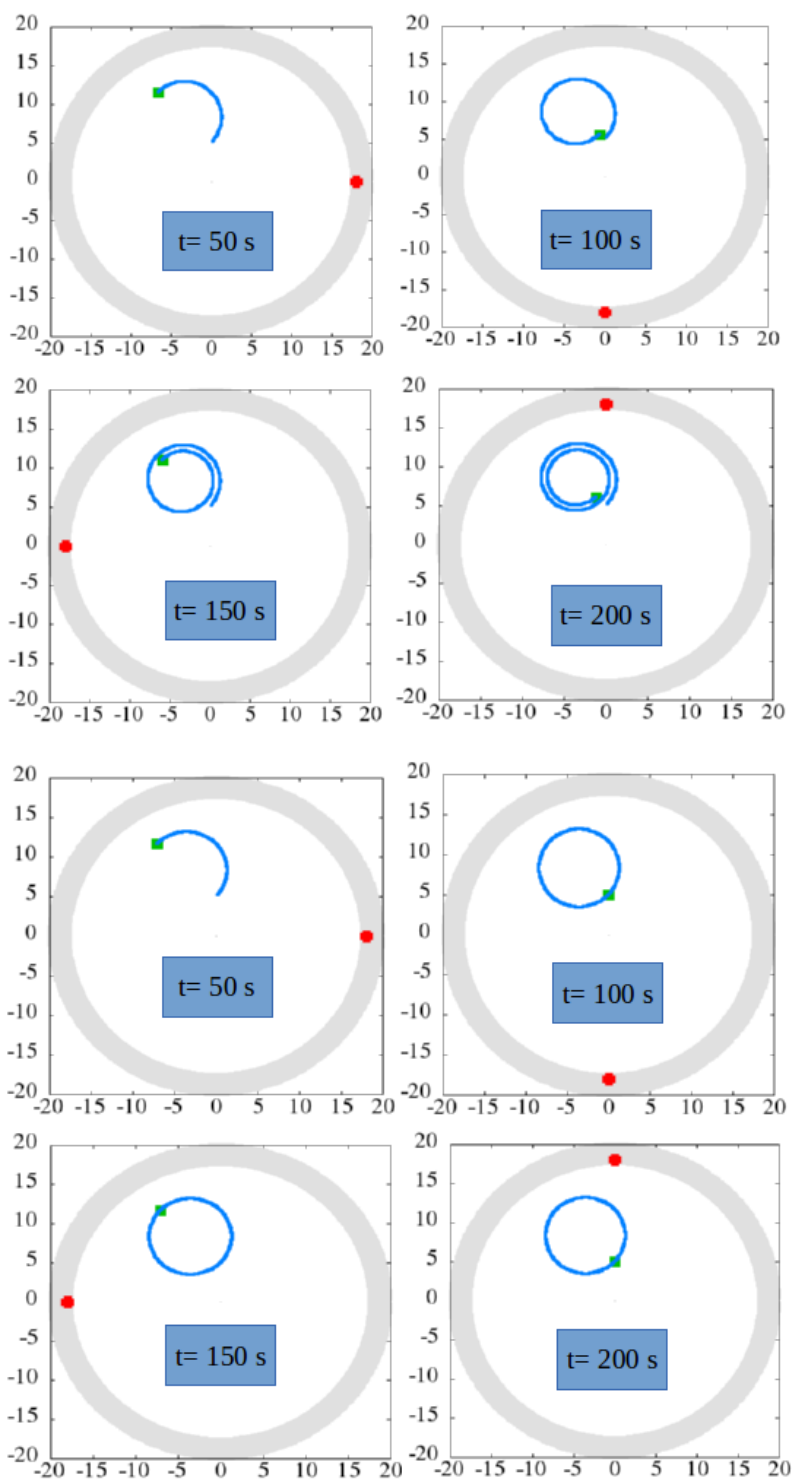

(a)

(b)

Figure 2.3.1: The parcel trajectory in a rotating frame. (a) Trajectory from the implicit Euler scheme. (b) Trajectory from trapezoidal scheme. Reference location in the fixed frame of reference is indicated with the dot location. 


\section{Chapter 3}

\section{Finite Difference for Diffusion Equation}

From this chapter onwards, we will consider various applications that apply mathematical models in the form of partial differential equations. On the first section, standard finite difference approximation for partial derivatives, and important numerical scheme terminologies will be resumed. Upon implementation of finite difference to a diffusive model, discussion about boundary conditions (Dirichlet or Neumann types) will get extra attention, as well as their physical interpretations.

\subsection{Finite Difference Method}

In principal, finite difference is a method to approximate a derivative with a ratio of differences. Implementation of finite difference to a differential equation will give us a difference equation. Basic finite difference formulas are as follows:

$$
\begin{array}{cl}
\text { Forward difference: } & \left.\frac{d f}{d x}\right|_{x_{j}}=\frac{f\left(x_{j}+\Delta x\right)-f\left(x_{j}\right)}{\Delta x}+\mathcal{O}(\Delta x),(3.1 .1) \\
\text { Backward difference: } & \left.\frac{d f}{d x}\right|_{x_{j}}=\frac{f\left(x_{j}\right)-f\left(x_{j}-\Delta x\right)}{\Delta x}+\mathcal{O}(\Delta x),(3.1 .2) \\
\text { Center difference: } & \left.\frac{d f}{d x}\right|_{x_{j}}=\frac{f\left(x_{j}+\Delta x\right)-f\left(x_{j}-\Delta x\right)}{2 \Delta x}+\mathcal{O}\left(\Delta x^{2}\right) .
\end{array}
$$

Task: Apply the Taylor expansion of $f(x)$ around $x_{j}$, and derive all finite difference formulas above. 
The order of the truncation term is called accuracy of the approximation formula. Hence, the forward and backward differences (3.1.1), (3.1.2) are of order one $\mathcal{O}(\Delta x)$, whereas center difference is of order two $\mathcal{O}\left(\Delta x^{2}\right)$. Formulas of finite difference approximation of higher order can be found in [6]. Also consult [6] for finite difference approximations for second order or third order derivatives. For partial derivatives of multi variable functions, similar/analogous formulas can be derived.

Task: Formulate forward, backward, and center differences to the partial derivatives $f_{x}, f_{t}$ of $f(x, t)$ at a grid point $\left(x_{j}, t_{n}\right)$.

Before we perform numerical computations, several important properties of difference equations for partial differential equation needs consideration. Consider a well-posed differential problem with an analytical solution $u(x, t)$, and the corresponding difference equation with numerical solution $u\left(x_{j}, t^{n}\right)$. The relation between differential equation and its difference equation is termed as consistency, while the relation between the analytical solution $u(x, t)$ and its approximate solution $u\left(x_{j}, t^{n}\right)$ is the concept of convergence. These connections are illustrated in the diagram in Figure 3.1.1.

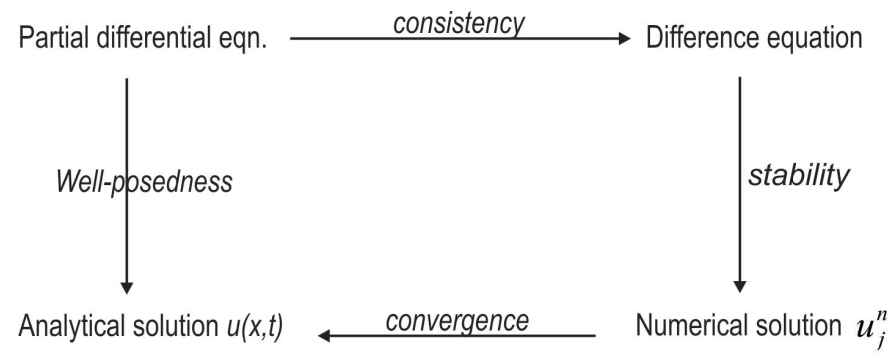

Figure 3.1.1: A connection diagram between the differential equation and its difference equation counterpart.

Starting form a difference equation, the modified differential equation (MDE) can be obtained if each terms in the difference equation are expressed in Taylor series at a base point. This effectively converts the difference equation back into a partial differential equation with an infinite number of additional terms. If the MDE tends to the original partial differential equation as grid spacing tends to zero, the difference equation is called consistent. Terms appearing in the MDE that do not appear in the original partial differential equation are called as the local discretization error or truncation 
error terms. Order of the local discretization error is indeed the order of the difference scheme.

Thus, several basic terminologies of a numerical scheme are resumed below:

- The order of a difference scheme is the order of the local discretization error.

- A difference equation is consistent with the partial differential equations if the difference between them vanishes as the size of $\Delta x, \Delta t$ tend to zero.

- A difference equation is stable if it produces a bounded solution.

- A finite difference method is convergent if the solution of the difference equation approaches the exact solution of the partial differential equation as $\Delta x, \Delta t$ tend to zero.

Lax equivalence theorem: It states that for a consistent finite difference method of a well-posed linear initial value problem, the method is convergent if and only if it is stable.

Proving convergence of a numerical solution is often difficult, it is much easier showing and of a difference scheme. Therefore in practice, Lax equivalence theorem is often used. To summarize, the concepts of consistency, order, stability and convergence must be considered when solving a partial differential equation by finite difference methods. Consistency and order can be determined from the modified differential equation. Stability can be investigated by applying the von Neumann method. This Von Neumann will be discussed later. Convergence can be ensured through Lax equivalence theorem by only checking the consistency and stability.

\subsection{Diffusion equation for heat conduction}

We begin by deriving the governing equation for heat conduction in a conductive bar with uniform cross-section. The bar is insulated laterally, so that the heat is only transferred to the left or right along the bar. In this case, we can conduct one-dimensional study. Consider a segment of the bar $[x, x+\Delta x]$, see Figure 3.2.1. Let $u(x, t)$ be the bar temperature at position $x$ and time $t$. Internal energy of that segment is $\rho c u \Delta x$, with $c$ and $\rho$ are specific heat and density of the bar, respectively. Let $f(u)$ denote 


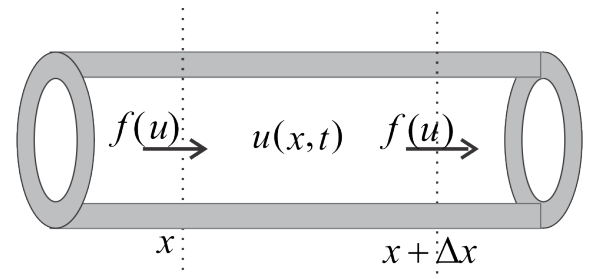

Figure 3.2.1: A segmen $[x, x+\Delta x]$ in the lateraly insulated bar.

the heat flux. Within time interval $\Delta t$, the change of internal energy in the segment is

$$
\rho c\left(\left.u\right|_{t+\Delta t}-\left.u\right|_{t}\right) \Delta x=\left(\left.f(u)\right|_{x}-\left.f(u)\right|_{x+\Delta x}\right) \Delta t
$$

Divide both sides with $\Delta t \Delta x$, and after taking the limits $\Delta x \rightarrow 0, \Delta t \rightarrow 0$, we obtain

$$
\rho c u_{t}+(f(u))_{x}=0 .
$$

Heat flows from high temperature to low temperature, and assume that heat flux obey Fick's law: the flux is proportional to temperature gradient, or $f(u)=-k u_{x}$, with a positive parameter $k$ denotes heat conductivities. Substituting this flux function into (3.2.1) will lead us to the following diffusion equation

$$
u_{t}=\kappa u_{x x}, \quad \text { with } \quad \kappa=\frac{k}{c \rho} .
$$

We have shown that diffusion equation is the governing equation for heat conduction. This equation serves as a model for dye distribution in a soluble liquid. The concentrated dye diffuses towards its surrounding, which has a lower concentration, with the rate proportional to the concentration gradient (again Fick's law), and you can proceed with similar fashion to derive the model. From now on, it is important to keep in mind the Fick's law for the flux, adopting it to the conservative equation will yield a diffusion equation. Furthermore, you may associate a diffusion equation as a model for distribution of heat or dye. This will help you to gain insight of the property of diffusion equation and its solution.

Consider a diffusion equation with thermal diffusivity $\kappa$

$$
\begin{array}{cl}
u_{t}=\kappa u_{x x}, & \text { for } 0<x<L, \quad t>0, \\
u(0, t)=0, u(L, t)=0, & \text { for } t>0, \\
u(x, 0)=f(x), & \text { for } 0<x<L,
\end{array}
$$


with the boundary conditions (3.2.4) and initial condition (3.2.5).

Boundary conditions (3.2.4) determine the value of $u$ at the boundaries $x=$ 0 and $x=L$, it is known as Dirichlet-type boundary. When the derivative of $u$ is known, for instance $u_{x}(0, t)=0$ then the boundary condition is said to be of Neumann-type. The homogeneous Neumann-boundary $u_{x}(0, t)=0$ has a physical interpretation that at $x=0$ there is no heat flux or $x=0$ is an isolated left boundary.

\section{Exercise 3.2.1.}

Consider the diffusion equation (3.2.3) with the initial condition (3.2.5). Without actually solving the equation, and only by considering the physical system, determine the final stage temperature $\lim _{t \rightarrow \infty} u(x, t)$ for each of the heat diffusion problem with boundary condition below.

- $u(0, t)=100, u(L, t)=0$

- $u(0, t)=100, u_{x}(L, t)=0$

- $u_{x}(0, t)=0, u_{x}(L, t)=0$, for this case assume $u(x, 0)=f(x)=x$

In the next section, we will discuss various finite difference schemes, starting from the simplest FTCS which is explicit and of order one, the BTCS implicit method, and finally the order two, the Crank-Nicolson scheme and theta method.

\subsection{The Euler explicit method}

In this section we will discuss a finite difference scheme to solve the diffusion equation $(3.2 .3,3.2 .4,3.2 .5)$. On the computational domain $\Omega=$ $\left\{(x, t) \in R^{2} \mid 0<x<L, t>0\right\}$, we introduce a grid, with $\Delta x$ denotes the spatial grid size and $\Delta t$ the time step. Let the spatial interval $[0, L]$ is devided into $N_{x}$ cells with grid size $\Delta x$, so there are $N_{x}+1$ grid points $0=x_{1}$, $x_{2}, \cdots, x_{j}=(j-1) \Delta x, \cdots, x_{N_{1}+1}=N_{x} \Delta x=L$. Hence, as illustrated on Figure 3.3.1 the computational domain consists grid points $\left(x_{j}, t^{n}\right)$ defined as

$$
\begin{aligned}
x_{j} & =(j-1) \Delta x, \quad j=1,2, \cdots, N_{x}+1, \quad \Delta x=L / N_{x}, \\
t^{n} & =(n-1) \Delta t, \quad n=1,2, \cdots
\end{aligned}
$$

The notation $u_{j}^{n}$ is used for the numerical approximation of $u\left(x_{j}, t^{n}\right)$. The given initial condition (3.2.5) means $u_{j}^{1}$, for $j=1, \cdots, N_{x}+1$ are known. 
And the left and right boundary conditions (3.2.4) are just $u_{1}^{n}=0$ and $u_{N x+1}^{n}=0$, for $n=1, \cdots, N t$. Further, with a numerical approach we need to determine $u_{j}^{n}$, for the remaining points, to be explicit $u_{j}^{n}$, with $j=2, \cdots, N_{x}, n=2, \cdots, N t$.

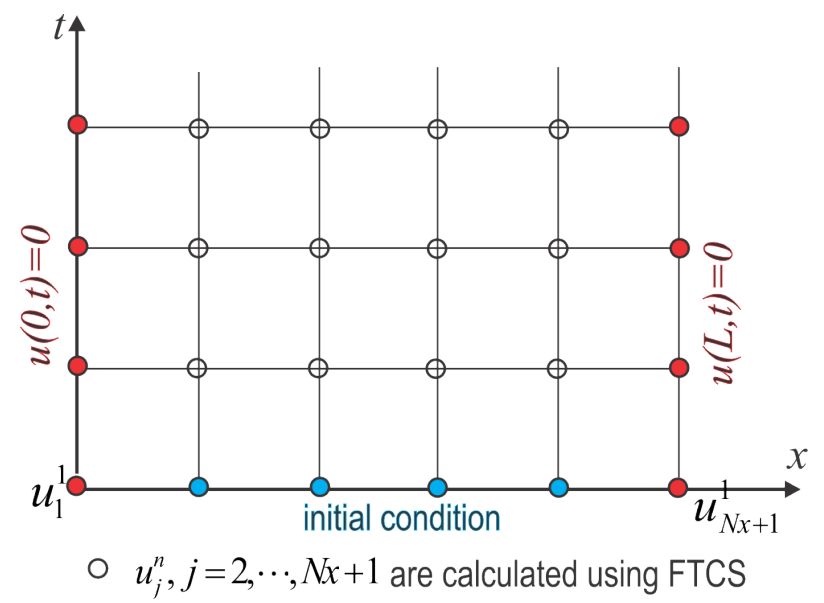

Figure 3.3.1: The computational domain with positions of initial condition, left and right boundary conditions.

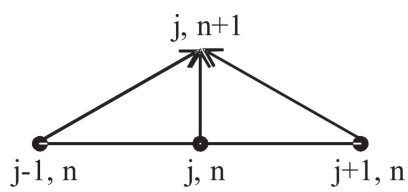

Figure 3.3.2: Stencil of the Euler explicit (or FTCS) method for the diffusion equation.

We approximate (3.2.3) by replacing the time derivative $\frac{\partial}{\partial t} u\left(x_{j}, t^{n}\right)$ with the forward difference approximation, and the space derivative $\frac{\partial^{2}}{\partial x^{2}} u\left(x_{j}, t^{n}\right)$ by the center difference approximation. By writing the numerical approximation of $u(x, t)$ at grid points $\left(x_{j}, t_{n}\right)$ as $u_{j}^{n}$, we obtain the difference equation of (3.2.3) as

$$
\frac{1}{\Delta t}\left(u_{j}^{n+1}-u_{j}^{n}\right)=\kappa \frac{1}{\Delta x^{2}}\left(u_{j+1}^{n}-2 u_{j}^{n}+u_{j-1}^{n}\right) .
$$


The scheme is simplified to

$$
u_{j}^{n+1}=u_{j}^{n}+S\left(u_{j+1}^{n}-2 u_{j}^{n}+u_{j-1}^{n}\right), \quad \text { with } \quad S=\kappa \frac{\Delta t}{\Delta x^{2}} .
$$

Using (3.3.4) we can compute $u_{j}^{n+1}$ if $u_{j+1}^{n}, u_{j}^{n}, u_{j-1}^{n}$ are known, this is expressed in the stencil of FTCS depicted in Figure 3.3.2. By adopting the initial condition $u_{j}^{1}=f\left(x_{j}\right)$ and boundary conditions $u_{1}^{n}=0, u_{N x+1}^{n}=0$, it is easy to check that we can compute all $u_{j}^{n+1}$ for $j=2, \cdots, N x, n=$ $1,2, \cdots, N t-1$ in the computational domain as in Figure 3.3.1.

\section{Stability}

To analyze the stability of the FTCS scheme, consider a numerical solution of the form

$$
u_{j}^{n}=U_{0} e^{i k x_{j}-i \psi t^{n}},
$$

with $k$ is the wave number, $\psi$ the wave frequency, and $U_{0}$ typical wave amplitude. Different representation is

$$
u_{j}^{n}=\lambda^{n} e^{i k x_{j}}, \text { with a complex number } \lambda=U_{0} e^{-i \psi \Delta t} .
$$

Substituting (3.3.6) into the scheme (3.3.4) gives us

$$
\lambda=1+S\left(e^{i k \Delta x}-2+e^{-i k \Delta x}\right)=1-4 S \sin ^{2}(k \Delta x / 2)
$$

Thus for each time step, the Fourier mode $e^{i k x_{j}}$ is amplified by the factor $\lambda$. Finite solution $u_{j}^{n}$ for all $n$ is obtained if $|\lambda| \leq 1$, which is satisfied if

$$
S=\kappa \frac{\Delta t}{\Delta x^{2}} \leq \frac{1}{2}
$$

Show that (3.3.7) is the stability condition of the Euler explicit scheme (3.3.4).

Be aware that condition (3.3.7) is the necessary condition for the stability of (3.3.3). Further, the above method is called the von Neumann stability method. If we only concern with the stability of a scheme, instead of (3.3.5) we can simply use the ansatz (3.3.6), and find a condition (in terms of the Courant number $C$ ) such that $|\lambda| \leq 1$.

$\%$ Pseudo code: FTCS for diffusion equation $u_{t}=\kappa u_{x x}$ $\%$ with homogeneous Dirichlet boundary condition) 
$\%$ Initialition

$\%$ Specify the computational domain: $0 \leq x \leq L, 0 \leq t \leq T$.

$\%$ Choose the grid size $\Delta x$ and $\Delta t$.

$\%$ Compute $N_{x}+1$ and $N_{t}$, the total number of grid points in $x$ and $t$.

$\%$ Define an initial condition $u(x, 0)$.

$u\left(1: N_{x}+1,1\right)=1$;

$\%$ Specify the Courant number $S$.

$S=\kappa * \Delta t / \Delta x^{2} ; \% S$ should satisfies stability cond. (3.3.7).

\% Compute $u\left(x_{j}, t^{n+1}\right)$ using (3.3.4).

for $n=1: N_{t}-1$

$u(1, n+1)=0 \%$ homogenous Dirichlet left boundary $u(0, t)=0$

for $j=2: N_{x}$

$u(j, n+1)=u(j, n)+S(u(j+1, n)-2 u(j, n)+u(j-1, n)) ; \%$ FTCS end

0

$u\left(N_{x}+1, n+1\right)=0 \%$ homogenous Dirichlet right boundary $u(L, t)=$

end

$\%$ Plot the result

xplot $=0: \Delta x: \mathrm{L}$

for $\mathrm{n}=1: N_{t}$

figure(1)

plot(xplot, $\mathrm{u}(:, \mathrm{n}+1))$

axis([0 L 0 1 $]$ )

grid on

pause $(0.1)$

end

\section{Exercise 3.3.1.}

1. As mentioned before, Local discretization error is the residual $d_{j}^{n}$ found as the difference between the differential equation (3.2.3) evaluated at grid point $\left(x_{j}, t^{n}\right)$ and the scheme (3.3.4). Show that

$d_{j}^{n}=\left.\frac{\kappa}{2}\left(\kappa \Delta t-\frac{1}{6} \Delta x^{2}\right) u_{4 x}\right|_{j} ^{n}$, which is of the order $\mathcal{O}\left(\Delta t, \Delta x^{2}\right)$. This first order error term is of diffusion type, and show that it is zero when the Courant number is $C=1 / 6$.

2. Use the numerical error as found in exercise above, and discuss the consistency of (3.3.4) as the discrete equation of (3.2.3).

3. The FTCS scheme for (3.2.3) is (3.3.4). By solving the difference scheme (3.3.4) is infact solving the modified differential equation of (3.2.3), 
identify this MDE and the first order error term. So, what additional (error) terms you got as you work with the scheme (3.3.4)?

4. Write a numerical code for the explicit Euler equation (3.3.4). Solve the diffusion equation (3.2.3) using boundary conditions (3.2.4) and initial condition $f(x)=1$, and reproduce Figure 3.3.3. Compare your numerical results with the analytical formula. Hint: adopt the separation variables method, the analytical solution of $(3.2 .3,3.2 .4)$ is

$$
u(x, t)=\sum_{n=1}^{\infty} b_{n} \exp ^{-(n \pi / L)^{2} t} \sin \left(\frac{n \pi x}{L}\right),
$$

with $b_{n}$ denotes the coefficients of sine-Fourier series of $f(x)$ on $0<$ $x<L$.

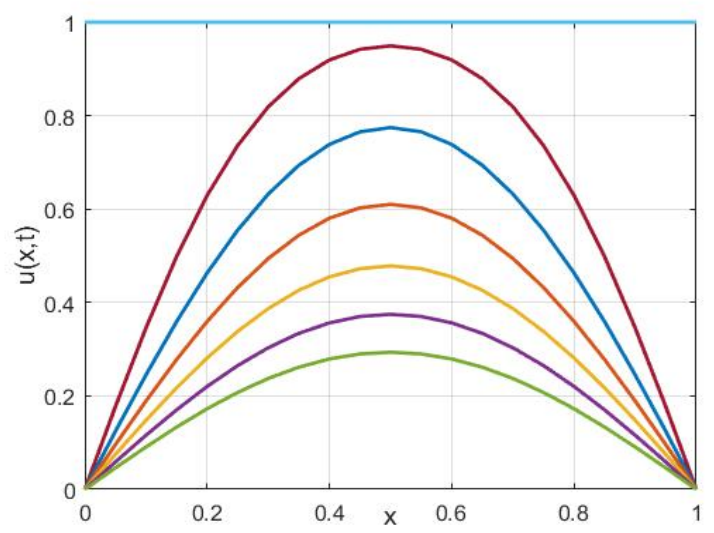

Figure 3.3.3: Plots of numerical solutions at subsequent times showing the decay of temperature because of zero temperatures at both ends, computed using parameters $\kappa=0.3, L=1, \Delta x=0.05, \Delta t=0.125 e-2$.

5. The FTCS method has an interesting feature when $S=\frac{1}{6}$. In this special case the $\mathcal{O}(\Delta t)$ and $\mathcal{O}\left(\Delta x^{2}\right)$ of the local discretization error $d_{j}^{n}$ cancels exactly, and the method becomes $\mathcal{O}\left(\Delta t^{2}, \Delta t \Delta x^{2}, \Delta x^{4}\right)$. Check the improved accuracy from your numerical results, by comparing the error of numerical results $u(x, 1)$ with the analytical, at several positions, i.e. $x=L / 4, L / 2,3 L / 4$.

6. Redo exercise no 2 using the non-trivial Dirichlet conditions $u(0, t)=$ $-1, u(L, t)=2$. Before conducting the simulation, predict how 
the temperature changes over time, your simulation should confirm your prediction.

\subsubsection{Modification for a Neumann boundary condition}

Condition (3.2.4) is of Dirichlet type. This condition is easily satisfied by taking $u_{1}^{n}=0$, and $u_{N_{x}+1}^{n}=0$, for all $n$, by simply implementing (3.3.4) for $j=2,3, \cdots, N_{x}$. But how if your physical model has a Neumann boundary condition?, like when your bar has an insulated left boundary with a mathematical expression

$$
u_{x}(0, t)=0 \text {. }
$$

Boundary condition (3.3.8) is known as a homogeneous Neumann boundary condition. Implementation of this boundary conditions needs extra attention, and here is the way how to implement them.

First, note that $u_{1}^{n}$, for $n=2, \cdots$ are not known, and this causes you can not compute $u_{1}^{2}$ using (3.3.4). If you do not have $u_{1}^{2}$, you cannot compute $u_{2}^{3}$ and $u_{2}^{3}$, etc. You will miss many points, and you will be in a real trouble! To avoid this, $u_{1}^{n}$ should be computed directly from $n=2,3, \cdots$. A way to do that is as follows. Starting from the given Neumann boundary condition (3.3.8), by applying the order-two center difference, we obtain the relation $u\left(-\Delta x, t^{n}\right)=u\left(\Delta x, t^{n}\right)$. Using this, we can then implement (3.3.4) directly from $\mathbf{j}=\mathbf{1}$, and this holds for all $n$.

\section{Exercise 3.3.2.}

1. Simulate how the temperature $u(x, t)$ changes as time progresses in the heat conduction problem below.

$$
\begin{array}{cl}
u_{t}=\kappa u_{x x}, & \text { for } 0<x<L, \quad t>0, \\
u_{x}(0, t)=0, u(L, t)=0, & \text { for } t>0, \\
u(x, 0)=1, & \text { for } 0<x<L
\end{array}
$$

Make sure that your numerical results produce physically relevant results. Remember that for heat conduction problem, a homogeneous Neumann boundary correspond to an insulated boundary.

2. Re-do the exercise above but now using $u_{x}(0, t)=1$. 


\subsection{Implicit method}

Implementation of the backward approximation for time derivative $\frac{\partial}{\partial t} u\left(x_{j}, t^{n+1}\right)$ and center difference approximation for $\frac{\partial^{2}}{\partial x^{2}} u\left(x_{j}, t^{n+1}\right)$ will give us the following implicit scheme

$$
\frac{1}{\Delta t}\left(u_{j}^{n+1}-u_{j}^{n}\right)=\kappa \frac{1}{\Delta x^{2}}\left(u_{j+1}^{n+1}-2 u_{j}^{n+1}+u_{j-1}^{n+1}\right) .
$$

The scheme is re-written as

$$
-S u_{j+1}^{n+1}+(1+2 S) u_{j}^{n+1}-S u_{j-1}^{n+1}=u_{j}^{n}, \quad \text { with } \quad S=\kappa \frac{\Delta t}{\Delta x^{2}} .
$$

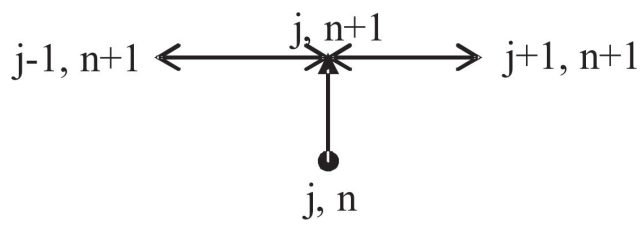

Figure 3.4.1: Stencil of the BTCS implicit method for the diffusion equation.

\section{Exercise 3.4.1.}

The numerical properties of the implicit scheme (3.4.2), and its implementation for solving (3.2.3-3.2.5).

1. Show that the local discretization error is $d_{j}^{n}=\left.\frac{\kappa}{2}\left(\kappa \Delta t-\frac{1}{12} \Delta x^{2}\right) u_{4 x}\right|_{j} ^{n}$, which is of order $\mathcal{O}\left(\Delta t, \Delta x^{2}\right)$.

2. Show that the scheme (3.4.2) is unconditionally stable.

3. In order to compute $u_{j}^{n}$, for every time step, we need to solve a system of equation $A \mathbf{u}^{n+1}=\mathbf{u}^{n}$, with

$$
A_{m \times m}=\left(\begin{array}{ccccc}
(1+2 S) & -S & 0 & \cdots & 0 \\
-S & (1+2 S) & -S & \cdots & 0 \\
\vdots & & & & \vdots \\
0 & \ldots & 0 & -S & (1+2 S)
\end{array}\right) .
$$

The vector $\mathbf{u}^{n}$ has $u_{j}^{n}$ as its element. What is the size of the square matrix $A$, is it $N_{x}-1, N_{x}$ or $N_{x}+1$ ? (Remember that if we deal with Dirichlet boundary condition (3.2.4), we already have $u_{1}^{n}=0$ and $u_{N_{x}+1}^{n}=0$ for all $n$.) 
4. Write a numerical code for solving (3.2.3-3.2.5) using the implicit scheme (3.4.2). Try several combination of $\Delta x$ and $\Delta t$ to make sure that the BTCS is an unconditionally stable scheme.

\subsection{Theta-method and Crank-Nicolson method}

In this section we will move on to the second order scheme. This second order scheme is a convex combination of the explicit scheme and implicit scheme. For a parameter $\theta$, with $0 \leq \theta \leq 1$, a combination of $\theta$-implicit and $(1-\theta)$ explicit will result in the following scheme

$\frac{1}{\Delta t}\left(u_{j}^{n+1}-u_{j}^{n}\right)=\kappa \frac{1-\theta}{\Delta x^{2}}\left(u_{j+1}^{n}-2 u_{j}^{n}+u_{j-1}^{n}\right)+\kappa \frac{\theta}{\Delta x^{2}}\left(u_{j+1}^{n+1}-2 u_{j}^{n+1}+u_{j-1}^{n+1}\right)$.

The scheme (3.5.1) with $\theta=\frac{1}{2}$ is called the Crank-Nicolson scheme. This is a second order scheme with accuracy $\mathcal{O}\left(\Delta t^{2}, \Delta x^{2}\right)$. Moreover, it is an unconditionally stable scheme.

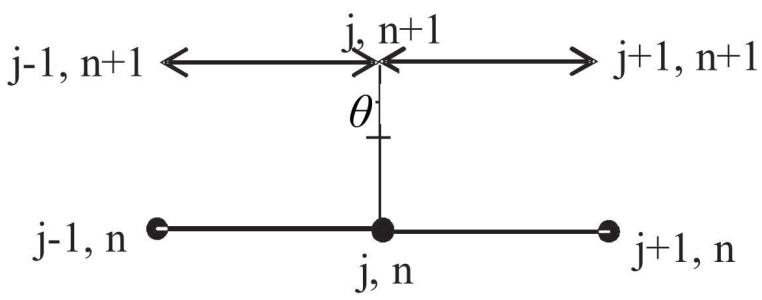

Figure 3.5.1: Stencil of the theta-method.

\section{Exercise 3.5.1.}

Numerical properties of the $\theta$-scheme (3.5.1)

1. Notice that when $\theta=0$ the scheme (3.4.2) becomes the FTCS explicit scheme, whereas when $\theta=1$ the scheme (3.4.2) becomes the BTCS implicit scheme.

2. Find the local discretization error $d_{j}^{n}$, and show that (3.5.1) is an order two scheme with accuracy $\mathcal{O}\left(\Delta t^{2}, \Delta x^{2}\right)$.

3. Show that the scheme (3.5.1) is unconditionally stable. 


\subsection{Stefan Problem}

Stefan problem refers to a phase change problem which involved moving boundary, such as solidification and melting. The main characteristic of Stefan problem is that interface location between two phases is unknown, thus it must be determined as part of Stefan solution. The Stefan problems are widely known since Josef Stefan studied the melting of polar ice cap and compare his results with measurement data around 1890. In early years, Stefan problem becomes an interesting topic in many fields such as natural science, industrial processes, and medical science. In industrial processes Stefan problems occur in metal solidification, food freezing, and ice production. Moreover, in medical science the Stefan problem can be found in cryosurgery, a process of destroying cancer cells using extremely cold temperatures. Several methods are developed to solve Stefan problems analytically and numerically. However, the analytical solutions are very limited even for one-dimensional problems.

Consider a one-dimensional container of length $l$ full of liquid with a freezing temperature $T_{m}$. Suppose the initial temperature of the liquid is $T_{L}$, which is higher than $T_{m}$. The left end of the liquid $x=0$ is maintained at temperature $T_{S}\left(<T_{m}\right)$ for all time $t$. Meanwhile, the right end $x=l$ is insulated. As a result, the solidification process starts from the left end. The left part of the liquid that solidifies will grow as time progresses. This process is well-known as Stefan problem. Here we assume that the material density $\rho$ is constant; and the thermo physical properties are: the latent heat is $L$, the respective specific heats of the liquid and solid $c_{L}$ and $c_{S}$, and the respective thermal conductivities of the liquid and solid $k_{L}$ and $k_{S}$.

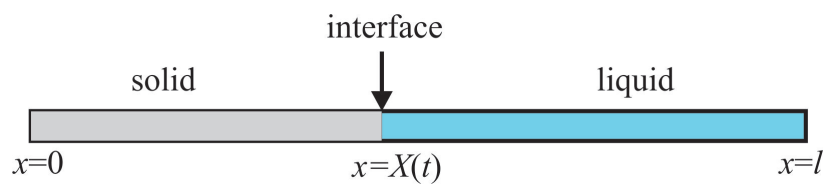

Figure 3.6.1: Description of one-dimensional Stefan problem.

Suppose $X(t)$ is the interface that separates the two regions at time $t$, such that $0 \leq x<X(t)$ is the solid region and $X(t)<x \leq l$ is the liquid region - cf. Figure 3.6.1. Our aim is to determine the temperature distribution $T(x, t)$ throughout the material, and the interface position $X(t)$. Heat conduction in solid and liquid regions obeys the respective diffusion equations

$$
\rho c_{S} T_{t}=k_{S} T_{x x}, \quad 0<x<X(t), \quad t>0 \quad \text { (solid) }
$$


and

$$
\rho c_{L} T_{t}=k_{L} T_{x x}, \quad X(t)<x<l, \quad t>0 \quad \text { (liquid). }
$$

At the interface $X(t)$, conservation of heat flux yields

$$
\rho L X^{\prime}(t)=k_{S} T_{x}\left(X(t)^{-}, t\right)-k_{L} T_{x}\left(X(t)^{+}, t\right), \quad t>0,
$$

where temperature at the interface is

$$
T(X(t), t)=T_{m}, \quad t>0 .
$$

Initial conditions and boundary conditions are given by

$$
\begin{array}{ll}
T(x, 0)=T_{L}\left(>T_{m}\right), & 0<x \leq l, \quad X(0)=0, \\
T(0, t)=T_{S}\left(<T_{m}\right), & T_{x}(l, t)=0, \quad t>0 .
\end{array}
$$

Hence, the governing equations for this problem are (3.6.1) and (3.6.2) with interface condition (3.6.3) and (3.6.4), initial conditions (5.2.2), and boundary conditions (3.6.6).

Further, the analytical solution of the above Stefan problem exists for the semi-infinite domain $x \geq 0$ is

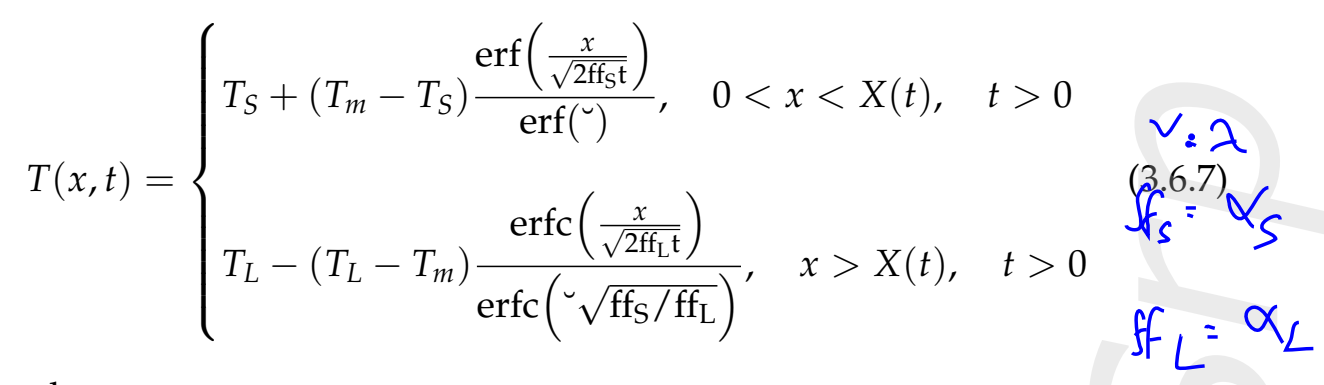

and

$$
X(t)=2 \lambda \sqrt{\alpha_{S} t}, \quad t>0,
$$

for derivation see [1]. Here the parameter $\lambda$ is a solution of the transcendental equation

where

$$
\frac{S t_{S}}{\exp \left(\lambda^{2}\right) \operatorname{erf}\left({ }^{\sim}\right)}-\frac{S t_{L}}{\exp \left(v^{2} \lambda^{2}\right) \operatorname{erf}\left({ }^{\circ}\right)}=\lambda \sqrt{\pi}
$$

$$
\alpha_{S}=\frac{k_{S}}{\rho c_{S}}, \quad S t_{S}=\frac{c_{S}\left(T_{m}-T_{S}\right)}{L}, \quad S t_{L}=\frac{c_{L}\left(T_{L}-T_{m}\right)}{L}, \quad v=\sqrt{\frac{\alpha_{S}}{\alpha_{L}}} .
$$


For a numerical solver of Stefan problem, the main difficulty lays in the emergence of moving interface that separates solid and liquid regions. Furthermore, it becomes complicated because we should solve both the heat conduction equation in the solid region and also in the liquid region, whereas the domain boundary moves. Apparently, formulating the governing equation in terms of energy (enthalpy) conservation turns out to be useful, and the inherited finite volume method appears to produce a very efficient scheme.

\section{Enthalpy formulation}

Let $F(x, t)$ denotes the internal energy in the system (Joule/kg, erg/gr). Internal energy of the system is related to the temperature through a relation as depicted in Figure 3.6.2 (left), where $c_{S}, c_{L}$ denotes specific heat in solid and liquid state, respectively, moreover $L$ denotes the latent heat. Inversely, temperature as a function of energy can be written explicitly as follows

$$
T(x, t)=\left\{\begin{array}{lrl}
T_{m}+\frac{E(x, t)}{\rho c_{S}}, & E(x, t) \leq 0 & \text { (solid) } \\
T_{m}, & 0<E(x, t)<\rho L & \text { (interface) } \\
T_{m}+\frac{E(x, t)-\rho L}{\rho c_{L}}, & E(x, t) \geq \rho L & \text { (liquid) }
\end{array}\right.
$$

and it is depicted in Figure 3.6.2 (right).
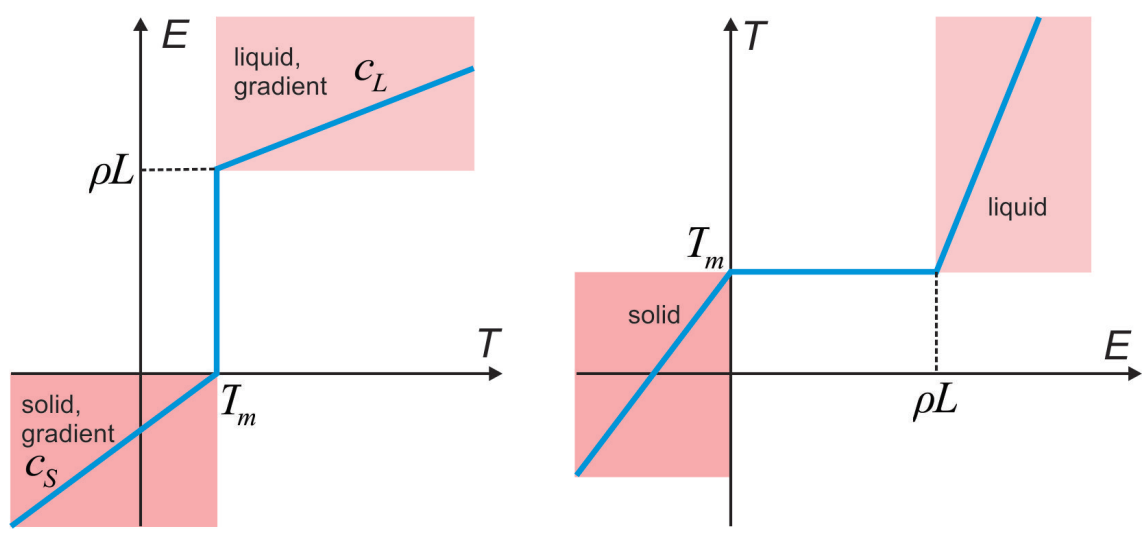

Figure 3.6.2: Relation between internal energy (enthalpy) $E$ with respect to temperature $T$ and its inverse temperature $T$ as a function of enthalpy $E$. 
Assuming there is no heat loss and there is no heat addition, the energy balance equation in discrete form reads

$$
\partial_{t} E+\partial_{x} Q=0
$$

where $Q$ denotes the energy flux. Adopting the Fick's law $Q=-k \partial_{x} T$, where $k=k_{S}$ or $k=k_{L}$ as in solid or liquid regions, respectively.

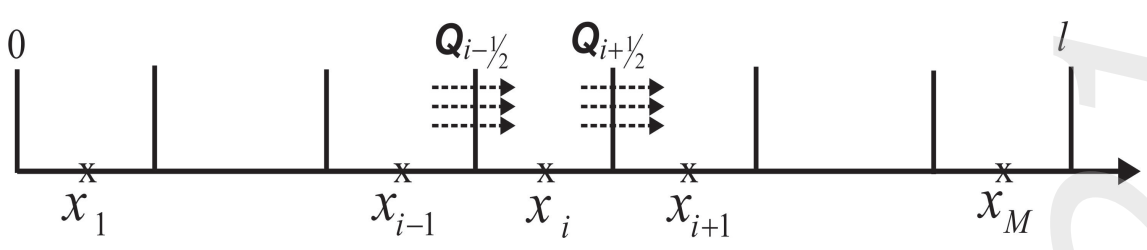

Figure 3.6.3: Partition of the computational domain $[0, l]$ in $N x$ control volumes.

The finite volume formulation of (3.6.12) start with dividing the fluid domain $[0, l]$ into $N x$ subregions called control volumes, $V_{i}, i=1,2, \cdots, N x$. Each control volume $V_{i}$ is associated with a point $x_{i}$ located at the midpoint of intervals, see Figure 3.6.3. Let $V_{i}=\left[x_{i-1 / 2}, x_{i+1 / 2}\right]$ be the $i-$ cell, a discrete form of energy balance in cell $V_{i}$ reads as

$$
E_{i}^{n+1}=E_{i}^{n}+\frac{\Delta t}{\Delta x}\left[Q_{i-1 / 2}^{n}-Q_{i+1 / 2}^{n}\right],
$$

with the approximate flux

$$
Q_{i-1 / 2}=-k \frac{T_{i}-T_{i-1}}{\Delta x},
$$

with $k=k_{S}$ or $k=k_{L}$ as in solid or liquid regions, respectively. This scheme can handle discontinuous solution automatically, as we would expect enthalpy $E$ to be discontinuous at the interface due to different thermal properties in each phase, for more information, see [16].

\section{Exercise 3.6.1.}

Implement the finite volume method and simulate the formation of ice in a liquid that has initial temperature $T(x, 0)=2^{0} \mathrm{C}$. Temperature of the left boundary is kept constant $T(0, t)=-1$, whereas the right boundary is insulated. In this case the solidification process will start from the left boundary, and as time progresses the ice grows in length. For this simulation, you can use the 'normalized' parameters $c_{S}=c_{L}=1$, freezing temperature $T_{m}=0$ and latent heat $L=1$. Plot the result in terms of temperature $T(x, t)$ as well as enthalpy $E(x, t)$. 

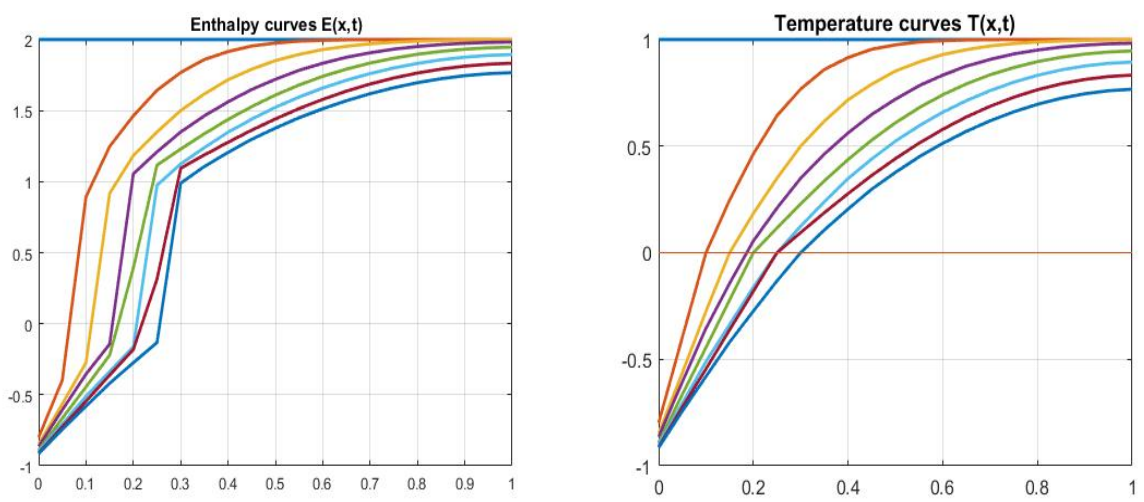

Figure 3.6.4: (Left) Snapshots of enthalpy curves $E(x, t)$. (Right) Snapshots of temperature curves $T(x, t)$. The interface between liquid and solid can be detected in two ways: from the temperature curve as a position with $T(x, t)=T_{m}$, and from the enthalpy curve as a position of discontinuous $E(x, t)$.

Note: make sure you understand the discrete formulation, as well as how to handle boundary conditions before implementing a code.

\% Pseudo code: Moving boundary Stefan problem using enthalpy

$\%$ Initialization

$\%$ Specify the computational domain: $0 \leq x \leq L, 0 \leq t \leq T$

$\%$ Choose the grid size $\Delta x$ and $\Delta t$.

$\%$ Compute $N_{x}+1$ and $N_{t}$, the total number of grid points in $x$ and $t$.

$\%$ Define an initial condition $E(x, 0)$.

$\%$ Set the values of global parameters $\rho, c_{S}, c_{L}, T_{m}$

global $\rho, c_{S}, c_{L}, T_{m}$

for $\mathrm{n}=1$ : $\mathrm{Nt}-1$

$\%$ Compute $E\left(x_{1}, t_{n+1}\right)$ using $(3.6 .13,3.6 .14)$,

$\%$ with left boundary condition $T(0, t)=T_{\text {left }}$.

$\mathrm{E}(1, \mathrm{n}+1)=\mathrm{E}(1, \mathrm{n})+\frac{d t}{d x^{2}}\left(2\left(-\phi(\mathrm{E}(1, \mathrm{n}))+T_{\text {left }}\right)+\phi(\mathrm{E}(2, \mathrm{n}))-\phi(\mathrm{E}(1, \mathrm{n}))\right) ;$

$\mathrm{T}(1, \mathrm{n}+1)=\phi(\mathrm{E}(1, \mathrm{n}+1))$;

$\%$ Compute $E\left(x_{j}, t_{n+1}\right)$ for $j=2 \cdots N x-1$ using $(3.6 .13,3.6 .14)$,

for $\mathrm{i}=2:(\mathrm{Nx}-1)$

$\mathrm{E}(\mathrm{j}, \mathrm{n}+1)=\mathrm{E}(\mathrm{j}, \mathrm{n})+\frac{d t}{d x^{2}}(-\phi(\mathrm{E}(\mathrm{j}, \mathrm{n}))+\phi(\mathrm{E}(\mathrm{j}-1, \mathrm{n})+\phi(\mathrm{E}(\mathrm{j}+1, \mathrm{n}))-\phi(\mathrm{E}(\mathrm{j}, \mathrm{n}))) ;$

$\mathrm{T}(\mathrm{j}, \mathrm{n}+1)=\phi(\mathrm{E}(\mathrm{j}, \mathrm{n}+1))$;

end 
$\%$ Compute $E\left(x_{N} x, t_{n+1}\right)$ for $j=2 \cdots N x-1$ using (3.6.13,

$\%$ with right boundary condition $T_{x}(l, t)=0$

$\mathrm{E}(\mathrm{Nx}, \mathrm{n}+1)==\mathrm{E}(\mathrm{Nx}, \mathrm{n})+\frac{d t}{d x^{2}}(-\phi(\mathrm{E}(\mathrm{Nx}, \mathrm{n}))+\phi(\mathrm{E}(\mathrm{Nx}-1, \mathrm{n}))) ; \mathrm{T}(\mathrm{Nx}, \mathrm{n}+1)=\phi(\mathrm{E}(\mathrm{Nx}, \mathrm{n}+1)) ;$ end

$\% \% \% \%$ On the same directory compose a function $T=\phi(E) \% \% \% \% \%$

$$
\begin{aligned}
& \text { function }[\mathrm{T}]=\phi(E) \\
& \text { global } \rho, c_{S}, c_{L}, T_{m} \\
& \text { if } E<0 \\
& \mathrm{~T}=T_{m}+\frac{1}{\rho c_{S}} E ; \\
& \text { elseif } E<1 \\
& \mathrm{~T}=T_{m} ; \\
& \text { else } \\
& \quad \mathrm{T}=T_{m}+\frac{1}{\rho c_{L}}(E-\rho L) \text {; } \\
& \text { end } \\
& \text { end }
\end{aligned}
$$

$\%$ Plotting temperature \& enthalpy

$$
\text { xplot }=0: \Delta x: \mathrm{L}
$$

for $\mathrm{n}=1: \mathrm{Nt}$

figure(1)

plot(xplot, $\mathrm{T}(:, \mathrm{n}+1))$

pause $(0.1)$

end

for $\mathrm{n}=1: \mathrm{Nt}$

figure(1)

plot(xplot, $\mathrm{E}(:, \mathrm{n}+1))$

pause $(0.1)$

end

\section{Exercise 3.6.2.}

This exercise is about the three phase changes of water $\mathrm{H}_{2} \mathrm{O}$. Formulate a function of temperature $T$ as a function of energy $E$ as given in figure above. Next we will simulate the phase change of water, which initially is at room temperature, and undergo a phase change due to the cold left boundary and hot right boundary. For the simulation, you can use a simplified function as follows.

$$
T=\left\{\begin{array}{lll}
E, & \text { if } E \leq 0 \\
0, & \text { if } 0<E 1 \\
E-1, & \text { if } 1<E \leq 2 \\
1, & \text { if } 2<E \leq 3 \\
E-3, & \text { if } 3<E & \text { (liquid) }
\end{array}\right.
$$




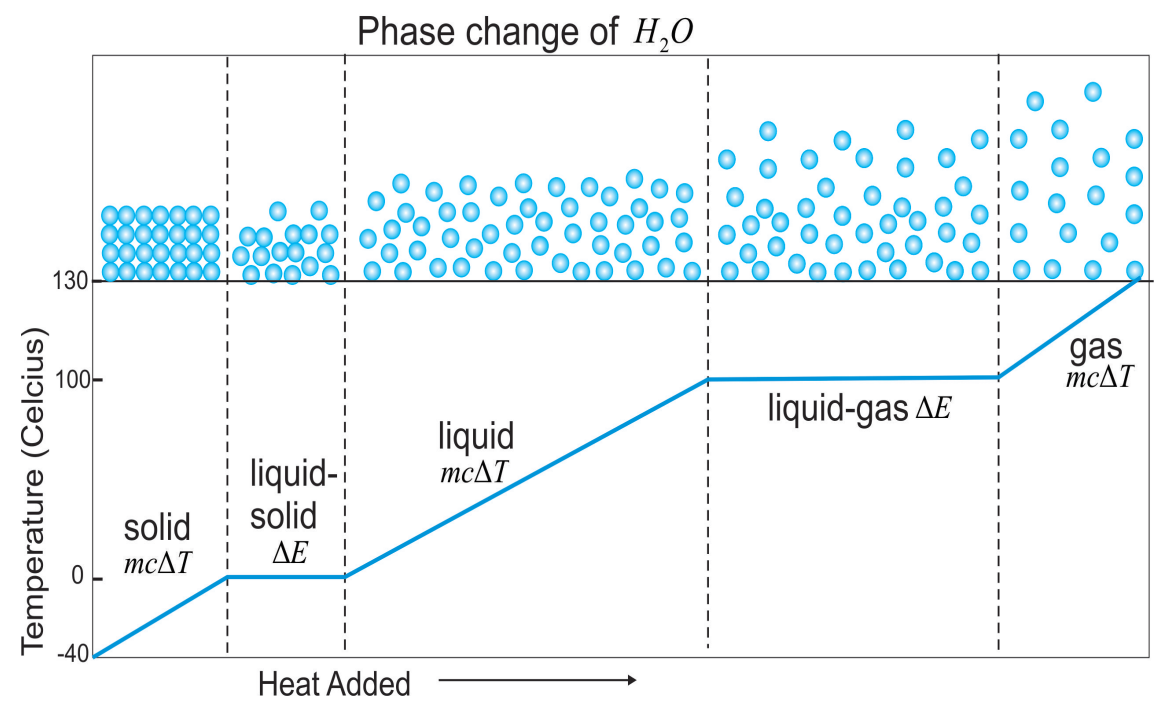

Figure 3.6.5: Three phase diagram of water $\mathrm{H}_{2} \mathrm{O}$.
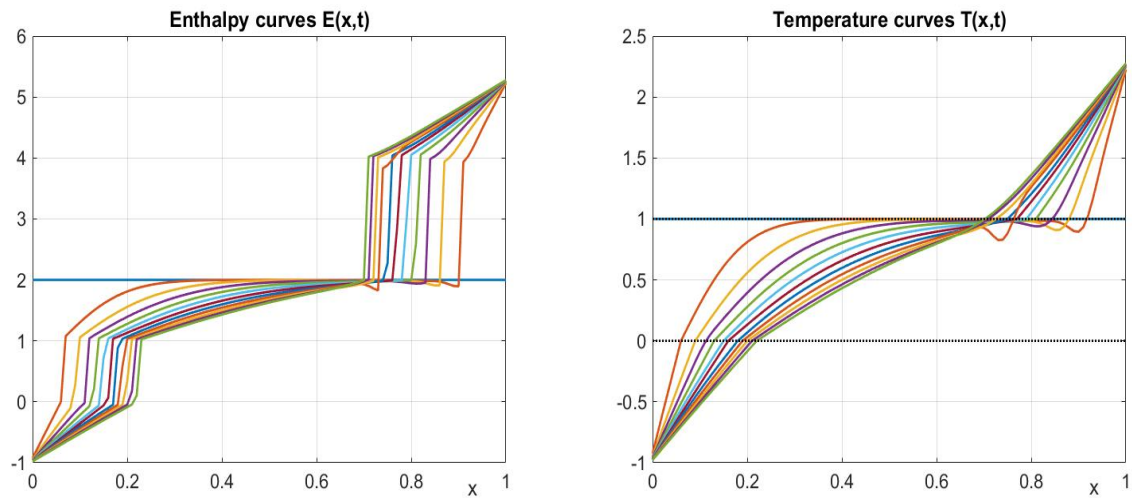

Figure 3.6.6: The snapshots of temperature and enthalpy curves on the changing phase proses of water.

Let the water column in a domain $0<x<l$ has an initial temperature $T(x, t)=2$. The left is maintained at low temperatures $T(0, t)=-1$, while the right is at high temperatures $T(l, t)=2.3$. Simulate the solidification processes starting from the left part, as well as the evaporation processes starting from the right part. For a water column with length $l=2$, the snapshots of temperature and enthalpy curves will typically looks like figures below. 
44 CHAPTER 3. FINITE DIFFERENCE FOR DIFFUSION EQUATION 


\section{Chapter 4}

\section{Finite Difference for Convection Equation}

In this section we discuss numerical methods for convection equation $u_{t}+$ $d u_{x}=0$. Analytical solution of the convection equation subjects to the initial condition $u(x, 0)=f(x), x \in R$, is given by $u(x, t)=f(x-d t)$. This solution represents a signal propagates undisturbed in shape with velocity $d$. The signal propagates to the right if $d>0$, and to the left if $d<0$. This analytical solution will be used to analyze numerical results. Numerical schemes describes in this chapter can be easily generalized for more complex problems, but of course, analysis are not as straight forward as this simplest case. Our discussion start with the method of characteristic, a method for finding the analytical solution of convection equation.

\subsection{Characteristic method}

Consider the convection equation with the initial condition below

$$
\begin{aligned}
& u_{t}+d u_{x}=0, \\
& u(x, 0)=f(x) .
\end{aligned}
$$

Its general solution will be formulated here using the method of characteristic. First, we notice that the left hand side of (4.1.1) can be written as

$$
\left(\begin{array}{l}
u_{x} \\
u_{t}
\end{array}\right) \cdot\left(\begin{array}{l}
d \\
1
\end{array}\right),
$$

which is a directional derivative of $u(x, t)$ in the direction of $\mathbf{v}=(d, 1)^{T}$, usually written as $D_{\mathbf{v}} u$. So, equation (4.1.1) can be written as

$$
D_{\mathbf{v}} u(x, t)=0 .
$$


Assuming $x$ is a function of $t$, we will find this characteristic curve $x(t)$. In this case, the characteristic curve has a constant gradient

$$
\frac{d x}{d t}=d .
$$

Since $d$ is a constant, the solution is a family of straight lines known as the characteristic curves:

$$
x=d t+k \text {, with } k \in \mathbb{R} .
$$

The governing equation dictates that directional derivatives $D_{\mathbf{v}} u$ is zero, hence we conclude that solution $u(x, t)$ of (4.1.1) should be constant along the characteristic $x(t)$ as given in (4.1.5). Using this principle, one can construct solution geometrically, see the following example.

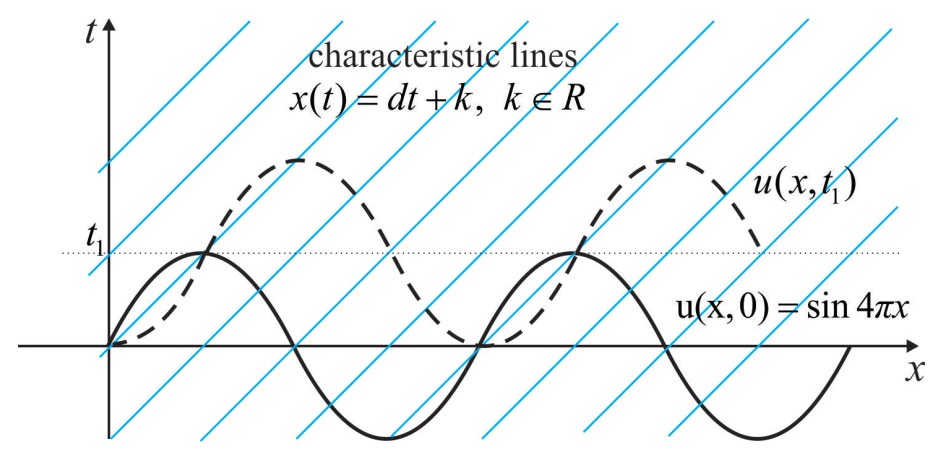

Figure 4.1.1: Characteristic lines $x(t)=d t+k, k \in \mathbb{R}$ used for constructing the analytical solution.

Example: Consider (4.1.1) with $d=1$ and initial condition $u(x, 0)=\sin (4 \pi x)$. Sketch the analytical solution by first drawing a family of characteristic lines. Then, the principle of solution $u(x, t)$ is constant along the characteristic is used to sketch solution at subsequent times. The result is illustrated in Figure (4.1.1).

This geometrically constructed solution can be used to check the numerical results that will be discussed later in Section 4.2. Note that from the above procedure we can only sketch the solution, and do not get the analytical solution formula yet. Analytical solution can be formulated as follows. Given the initial condition (4.1.2), the analytical solution reads $u(x, t)=f(k)$, with a parameter $k$ that should be specified by the characteristic line that passes through $(x, t)$. For an arbitrary point $(x, t)$ there 
should be a unique ${ }^{1}$ characteristic line that passes through this point, say $x-d t=k$. Hence, the analytical formula of (4.1.1) with initial condition (4.1.2) reads as $u(x, t)=f(k)=f(x-d t)$.

\subsection{Upwind method}

In this section we will discussed about numerical schemes suitable for solving the convection equation with initial condition

$$
\begin{array}{cll}
u_{t}+d u_{x}=0, & \text { for } 0<x<L, \quad t>0, \\
\text { (Initial condition) } \quad u(x, 0)=f(x), & \text { for } 0<x<L .
\end{array}
$$

Here, we restrict to the case of $d>0$, in which the solution is $u(x, t)=$ $f(x-d t)$, a right propagating wave. The simplest finite difference scheme discussed first is the upwind method. Upwind method is a first order scheme: Forward Time Backward Space (FTBS) or Forward Time Forward Space (FTFS). Which scheme to be implemented depends on the flow direction. For the right running flow $d>0$, the suitable scheme is FTBS, whereas for the left running flow $d<0$, one should apply FTFS.

Here we consider $(4.2 .1,4.2 .2)$ with $d>0$. On the computational domain $\Omega=\left\{(x, t) \in R^{2} \mid 0<x<L, t>0\right\}$, we introduce a grid, with the spatial grid size $\Delta x$ and time step $\Delta t$. The grid points $\left(x_{j}, t^{n}\right)$ are defined as

$$
\begin{aligned}
x_{j} & =(j-1) \Delta x \quad j=1,2, \cdots, N_{x}+1, \quad \Delta x=L / N_{x}, \\
t^{n} & =(n-1) \Delta t \quad n=1,2, \cdots
\end{aligned}
$$

In this case $d>0$, which means right running signal, therefore here we implement the Forward Time Backward Space (FTBS) method. Applying FTBS to the convection equation (4.2.1) will yield

$$
\frac{u_{j}^{n+1}-u_{j}^{n}}{\Delta t}+d \frac{u_{j}^{n}-u_{j-1}^{n}}{\Delta x}=0 .
$$

or explicitly

$$
u_{j}^{n+1}=(1-C) u_{j}^{n}+C u_{j-1}^{n},
$$

with $C \equiv \frac{d \triangle t}{\Delta x}$ a Courant number. A stencil for this method is depicted in Figure 4.2.1. In the FTBS scheme, the approximation adopted are of order

\footnotetext{
${ }^{1}$ This fact is directly related to the existence and uniqueness of an initial value problem with differential equation (4.1.4).
} 
one, therefore we can guess already that this upwind method has an accuracy of $\mathcal{O}(\triangle t, \triangle x)$.

Task: Prove that the FTBS scheme is an order one scheme, i.e. you need to show that the local discretization error is of order $\mathcal{O}(\triangle t, \triangle x)$.

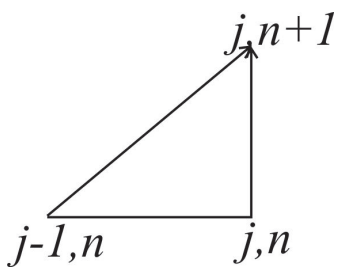

Figure 4.2.1: Stencil of the upwind method.

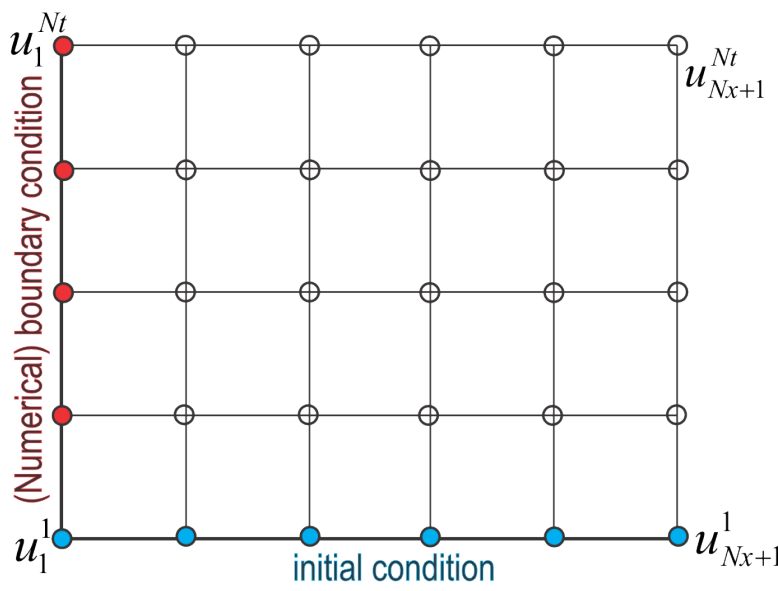

$\bigcirc u_{i}^{n}, j=2, \cdots, N x+1$ are calculated using FTBS

Figure 4.2.2: Computational domain with locations of initial and boundary conditions, and computed values $u_{j}^{n}, j=2, \cdots, N_{x}+1, n=2, \cdots, N t$.

From the initial condition (4.2.2), values of $u_{j}^{1}$, for $j=1,2, \cdots, N_{x}+1$ is known, see Figure 4.2.2. Using this initial values, it is clear that the FTBS scheme with stencil as in Figure 4.2.1 can be used to compute $u_{j}^{2}$, for $j=2, \cdots, N_{x}+1$, but not $u_{1}^{2}$. Here, we need a value of $u_{1}^{2}$, infact we need $u_{1}^{n}$, for all $n=2,3, \cdots$, see Figure 4.2.2. Note that, this left boundary condition is not provided by the original problem, but it is required in 
the implementation of the upwind FTBS scheme. This type of boundary condition is called a numerical boundary condition. For the first try, one can just take zero left boundary $u(0, t)=0$ or $u_{1}^{n}=0$, for all $n$. Later on you can experiment with various left boundary $u(0, t)=g(t)$, with $g(t)$ plays a role as signal that enters from the left boundary. By doing this, we can compute $u_{j}^{n}$, for $j=1, \cdots, N_{x}+1$, for all $n$ using the FTBS scheme (4.2.6).

$\%$ Pseudo code: FTBS for convection equation $u_{t}+d u_{x}=0$

$\%$ Initialition

$\%$ Specify the computational domain:

$\%$ spatial $0 \leq x \leq L$ and time $0 \leq t \leq T$.

$\%$ Choose the grid size $\Delta x$ and $\Delta t$.

$\%$ Compute $N_{x}+1$ and $N_{t}$, the total number of grid points in $x$ and $t$.

$\%$ Define an initial condition $u(x, 0)$.

$u\left(1: N_{x}+1,1\right)=0 ; u(1: 4,1)=1 ; \%$ a square wave initial condition.

$\%$ Specify the value of Courant number $C$.

$C=d * \Delta t / \Delta x ; \%$ Stability condition: $C \leq 1$.

$\%$ Compute $u\left(x_{j}, t_{n+1}\right)$ using (4.2.6).

for $n=1: N_{t}$

$u(1, n+1)=0 ; \%$ Zero left boundary

for $j=2: N_{x}+1$

$u(j, n+1)=(1-C) u(j, n)+C u(j-1, n) ;$

end

end

$\%$ Plot the result

figure(1)

surf( $\left(\mathrm{u}^{\prime}\right) \%$ Matlab command for surface plot, find out the difference with $\operatorname{surf}(\mathrm{u})$.

drawnow

Further, we investigate the stability condition of the upwind scheme using the von Neumann method, which is done by substituting the following ansatz $u_{j}^{n}=\lambda^{n} e^{i k x_{j}}$, into (4.2.6) to find the following relation

$$
\lambda=1-2 C \sin ^{2}(k \Delta x / 2)-i C \sin (k \Delta x) .
$$

A scheme is stable if $\left|u_{j}^{n}\right|$ is finite for all $j$ and $n$, since $\left|e^{i k x_{j}}\right|=1$, stability of the FTBS scheme is guaranteed only if $|\lambda| \leq 1$ for all $k \Delta x$. Then, the stability condition of the upwind scheme is $0<\frac{\bar{d} \Delta t}{\Delta x} \leq 1$. It is often stated that for a stable upwind scheme the Courant number should be less than unity. 
Further discussion, the stability condition $0<C=d \frac{\Delta t}{\Delta x} \leq 1$ has an interesting geometrical interpretation. When $d \frac{\Delta t}{\Delta x}=1$, the FTBS scheme (4.2.6) becomes $u_{j}^{n+1}=u_{j-1}^{n}$. In this case, $d \Delta t=\Delta x$ and the line that connects $\left(x_{j}, t^{n+1}\right)$ and $\left(x_{j-1}, t^{n}\right)$ is indeed the characteristic line with gradient $1 / d$, plotted as a solid line in Figure 4.2.3. When the Courant number equals to one $C=1$, the numerical error is zero (to be discussed in Exercise 4.2.1 number 1), and hence the upwind scheme produces the analytical result. Further, in the case of $d \frac{\Delta t}{\Delta x}<1$, the FTBS scheme (4.2.6) forms a convex combination of values $u_{j}^{n}$ and $u_{j-1}^{n}$, illustrated in Figure 4.2.3 by the dashed line. Therefore we can also interpret the stability condition $d \frac{\Delta t}{\Delta x} \leq 1$ as follows: for a stable scheme, the numerical speed cannot exceed the analytical signal speed.

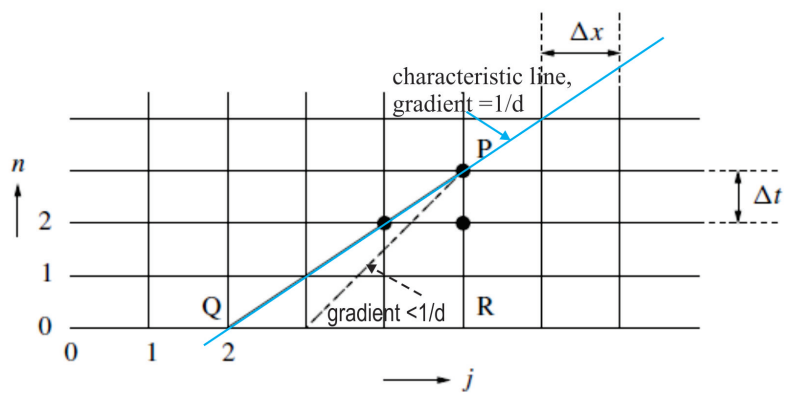

Figure 4.2.3: Stability condition $C=\frac{d \Delta t}{\Delta x} \leq 1$ with a geometrical interpretation on the computational mesh.

\section{Exercise 4.2.1.}

1. Show that the first order term of numerical error of the upwind FTBS scheme is $\left.\frac{1}{2} d(d \Delta t-\Delta x) u_{x x}\right|_{j} ^{n}$, which is a difussion error of order one. Find the second order term, also the third. If the Courant number $C=d \frac{\Delta t}{\Delta x}=1$, show that the first order error is zero. In fact, the second, the third, and all the rest of numerical error terms are also zero if $C=1$, see [6]. Hence, if $C=1$ the upwind scheme produces the analytical result.

2. Use the numerical error as found in exercise above, and discuss the consistency of (4.2.6) as the discrete equation of (4.2.1).

3. Write a numerical code for the FTBS scheme (4.2.6). For the first simulation take a combination of $\Delta t$ and $\Delta x$ such that the Courant 
number $C=d \frac{\Delta t}{\Delta x}$ is exactly one, since $d>0$. Using this set up, you should get a square wave propagates to the right. Make sure your wave propagates with the correct velocity $d$. Also check that the wave influx that enters from the left side of the domain matches with your chosen numerical left boundary.

4. Perform another simulation using different $\Delta t$ in such a way that $C=1.1$, explain the result. Since the Courant number is larger than one, your numerical solution should be unstable, in which the numerical value $u_{j}^{n}$ increases as time progresses.

5. Conduct another computation using different $\Delta t$ in such a way that the Courant number $C$ is strictly less than one. Your simulation should demonstrate that very soon discontinuity of the square wave disappear, due to diffusion effect. This is a direct consequence of the diffusive error term $\left.\frac{1}{2} d(d \Delta t-\Delta x) u_{x x}\right|_{j} ^{n}$. For a stable scheme, this damping effect will only be observed when $C=d \frac{\Delta t}{\Delta x}$ strictly less than one. Please check this aspect using your code.

6. The upwind scheme for (4.2.1) is (4.2.6). By solving the difference scheme (4.2.1) is infact solving the modified differential equation of (4.2.1), identify this MDE and the first order error term. So, what an additional (error) term you got as you work with the scheme (4.2.6)? Connect this with your findings of the previous exercise.

7. Implement the upwind scheme to solve the convection equation $u_{t}+$ $u_{x}=0$ for $0<x<1$ subject to initial condition $u(x, 0)=\sin 8 \pi x$. For computation use $\Delta x=1.25 \times 10^{-2}$ and $\Delta t=10^{-2}$. Plot the numerical result at time $t=1$ together with the exact result, and reproduce Figure 4.2.4 (you need to implement a suitable left boundary condition in order to get this figure). This computation is conducted using $C=\frac{d \Delta t}{\Delta x}=0.8$, and the result clearly shown a significant damping error. Less damping scheme will be obtained if the Courant number is closer to one. Please check this aspect.

Discussion: Find a suitable scheme for the convection equation $u_{t}+d u_{x}=$ 0 with $d<0$. For the chosen numerical scheme, what kind of 'numerical' boundary condition is needed? Repeat the last exercise above but now for $u_{t}-u_{x}=0$. What kind of boundary condition should be implemented in order to simulate a numerical sine wave propagating to the left? Modify your previous code to simulate this. 


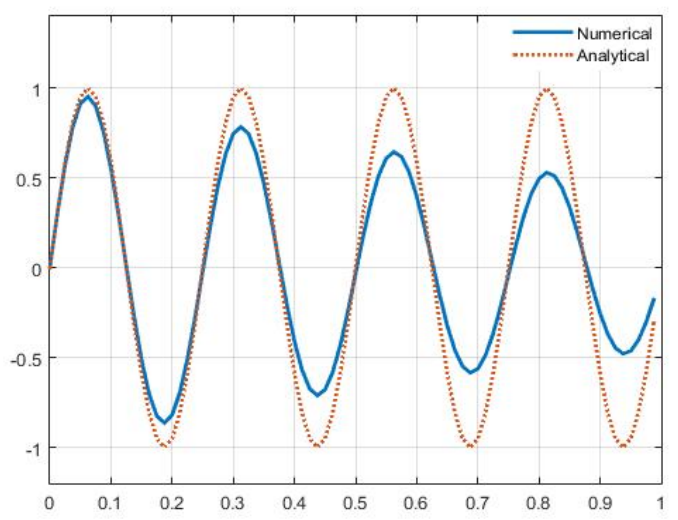

Figure 4.2.4: Numerical approximation of a sine wave at time $t=1 \mathrm{com}$ puted using the upwind scheme.

\subsection{Error in numerical solutions}

In this section, we will further analyze the error of a numerical solution. Basically, numerical solution may differ from the analytical solution from two sources: amplitude error and phase error. These error can be analyzed from the representation of numerical solution as follows

$$
u_{j}^{n}=\lambda^{n} e^{i k x_{j}}, \text { with } \lambda=U_{0} e^{-i \psi \Delta t} .
$$

This numerical solution has phase velocity $\frac{\psi}{k}$, denoted as $\beta=\frac{\psi}{k}$. This numerical solution (4.3.1) is expected to approximate the analytical solution of the convection equation $u_{t}+d u_{x}=0$ in the form

$$
u(x, t)=U_{0} e^{i(k x-\omega t)}, \text { with } \omega=d k .
$$

This analytical solution has phase velocity $d$. Comparing the numerical solution (4.3.1) with the analytical (4.3.2), we can conclude that

$$
\begin{array}{cl}
\text { amplitude error } & \varepsilon_{a}=1-|\lambda|, \\
\text { relative phase error } & \varepsilon_{f}=\frac{d k \Delta t-\beta k \Delta t}{d k \Delta t}=1-\frac{\beta}{d} .
\end{array}
$$

At every time step, the numerical solution will be multiplied by $\lambda$. Hence, in order to be finite the amplitude error should satisfy $0 \leq \varepsilon_{a}<1$. This condition will yield a stability condition for the numerical scheme, as demonstrated in Section 4.2. Moreover, if $\varepsilon_{a}>0$ the numerical solution contains 
damping error. Often, a numerical method contain damping error, so a damping free numerical scheme (happened if $\varepsilon_{a}=0$ ) is expected. If the relative phase error $\varepsilon_{f}>0(\beta<d)$ the numerical solution will lag behind the exact solution. Combining the definitions of $\varepsilon_{a}$ and $\varepsilon_{f}$ with the representation (4.3.1), we find the following relation between the exact and numerical values:

$$
u_{j}^{n}=\left(\left(1-\varepsilon_{a}\right) \exp \left(i \varepsilon_{f} d k \Delta t\right)\right)^{n} u\left(x_{j}, t^{n}\right) .
$$

Now we apply this to the upwind scheme of (4.2.1). To begin, let us substitute (4.3.1) to the scheme (4.2.6) and derive the following relation

$$
\lambda=1-C\left(1-e^{-i k \Delta x}\right)=1-2 C \sin ^{2}(k \Delta x / 2)-i C \sin (k \Delta x) .
$$

Next, from the polar representation of $\lambda$ we can get

$$
|\lambda|^{2}=1-4 C(1-C) \sin ^{2}(k \Delta x / 2), \quad \tan \psi=\frac{C \sin (k \Delta x)}{1-2 C \sin ^{2}(k \Delta x / 2)},
$$

From the first relation, we can determine the amplitude error. In order to derive an expression for the phase error, we have to invert the second relation using $\tan ^{-1}: \mathbb{R} \rightarrow[0, \pi)$, explicitly

$$
\tan ^{-1}(q):= \begin{cases}\arctan q, & \text { if } q \geq 0 \\ \arctan q+\pi, & \text { if } q<0\end{cases}
$$

Further investigation about this error analysis can be obtained for instance in the textbook [10], Subsection 13.2.

\section{Exercise 4.3.1.}

1. (A naive method) Another idea is to implement the forward time and center space (FTCS) approximation to yield

$$
\frac{u_{j}^{n+1}-u_{j}^{n}}{\Delta t}=-d \frac{u_{j+1}^{n}-u_{j-1}^{n}}{2 \Delta x} .
$$

This scheme seems preferable for two reasons: it may accommodate both cases $d>0$ and $d<0$, and it has second order accuracy in space: FTCS scheme is of order $\mathcal{O}\left(\Delta t, \Delta x^{2}\right)$. Implement stability analysis and argue that this FTCS scheme is always unstable, which means this scheme is useless. 
2. A remedy of the FTCS scheme above is called the Lax scheme, which simply replace $u_{j}^{n}$ in (4.3.7) with the average $\frac{1}{2}\left(u_{j+1}^{n}+u_{j-1}^{n}\right)$ to yield

$$
u_{j}^{n+1}=\frac{1}{2}\left(u_{j+1}^{n}+u_{j-1}^{n}\right)-d \frac{\Delta t}{\Delta x} \frac{1}{2}\left(u_{j+1}^{n}-u_{j-1}^{n}\right) .
$$

Show that this scheme is of order $\mathcal{O}\left(\Delta t, \Delta x^{2}\right)$, moreover this scheme is stable under the following condition $0<\frac{d \Delta t}{\Delta x} \leq 1$.

3. Upwind scheme for an arbitrary $d$.

$$
\frac{1}{\Delta t}\left(u_{j}^{n+1}-u_{j}^{n}\right)=-\frac{d^{+}}{\Delta x}\left(u_{j}^{n}-u_{j-1}^{n}\right)-\frac{d^{-}}{\Delta x}\left(u_{j+1}^{n}-u_{j}^{n}\right),
$$

with $d^{+} \equiv \max (d, 0)$ dan $d^{-} \equiv \min (d, 0)$. Implement this scheme using the initial condition: a hump with peak located in the middle of the domain. Take a positif value of $d$ and simulate the propagation of the initial hump to the right. Then, without changing the scheme, take the negative value of $d$, and show that the initial hump is now propagates to the left.

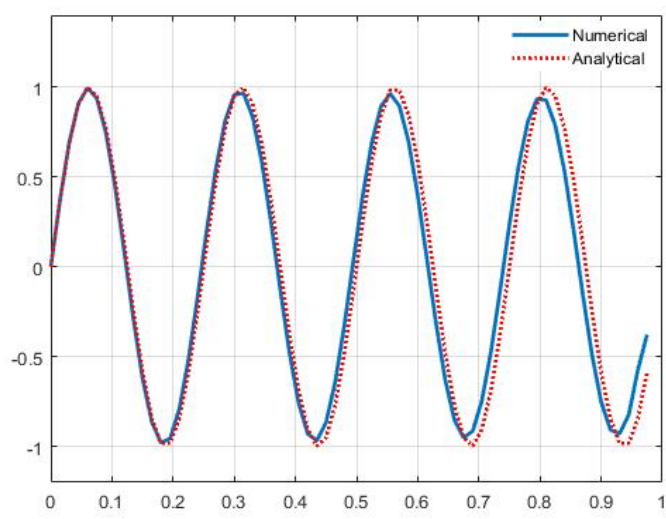

Figure 4.3.1: Numerical solution of the Lax-Wendroff scheme together with the analytical solution at $t=1$.

4. The Lax-Wendroff scheme of the convection equation $u_{t}+d u_{x}=0$ reads as

$$
u_{j}^{n+1}=u_{j}^{n}-\frac{1}{2} C\left(u_{j+1}^{n}-u_{j-1}^{n}\right)+\frac{1}{2} C^{2}\left(u_{j+1}^{n}-2 u_{j}^{n}+u_{j-1}^{n}\right) .
$$


Show that this Lax-Wendroff scheme is a second order scheme with accuracy $\mathcal{O}\left(\Delta t^{2}, \Delta x^{2}\right)$. Implement this scheme to solve $u_{t}+u_{x}=0$ subject to initial condition $u(x, 0)=\sin 8 \pi x$. Use $\Delta x=0.015$ and $\Delta t=0.01$, and plot the numerical scheme together with the exact solution at time $t=1$ as shown in Figure 4.3.9. Think of a suitable left boundary condition to produce this figure. When we compare with the upwind scheme, the Lax-Wendroff scheme clearly has less damping error, but the phase error is clearly visible.

5. Phase error of the Lax-Wendroff scheme Conduct an error analysis of this Lax-Wendroff scheme by substituting $u_{j}^{n}=\lambda^{n} e^{i k x_{j}}$ into (4.3.9) to find

$$
\lambda=1-2 C^{2} \sin ^{2}(k \Delta x / 2)-i C \sin (k \Delta x) .
$$

Calculate $|\lambda|$ and derive the following Lax-Wendroff stability condition $0<\frac{d \Delta t}{\Delta x} \leq 1$. Also investigate the phase error using $\varepsilon_{f}$.

6. Implement the Lax-Wendroff method to solve $u_{t}+0.8 u_{x}=0$, on $0<$ $x<1, t>0$, with a square wave initial condition. For computation, use $\Delta x=\Delta t=0.01$, which yields a Courant number $C=0.8$. Simulate wave propagation and plot numerical solution together with the exact solution, as shown in the figure below. The Lax-Wendroff produces a dispersion error in terms of wiggle near wave discontinuities, in comparison with numerical damping error from the implementation of the upwind (FTBS) method. These errors appears because in this case the Courant number is less than one.

Another second order scheme is the leapfrog scheme. Numerical error of this scheme is dispersion error. Interested reader may consult references $[6,10]$ for further study. 

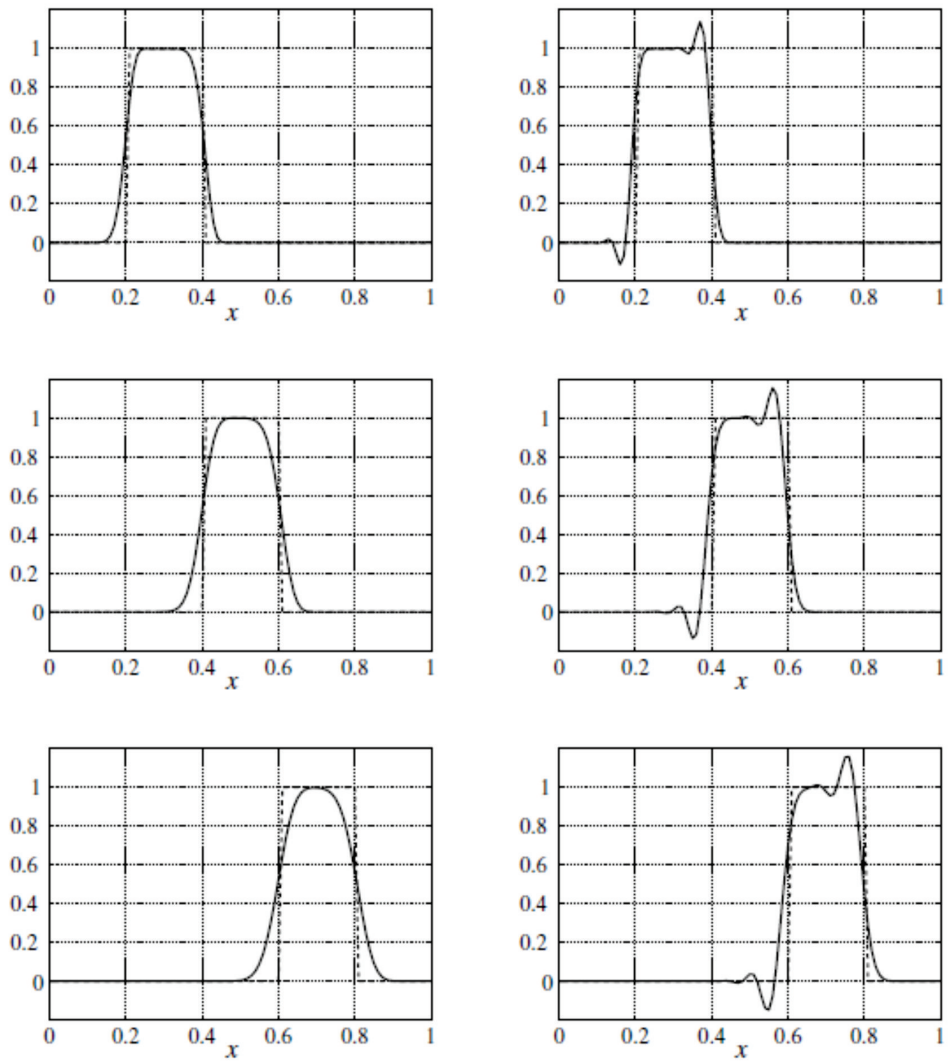

Figure 4.3.2: Comparison of exact solution with the upwind numerical method (left), and the Lax-Wendroff method (right). 


\section{Chapter 5}

\section{Conservative Problems \& Finite Volume Method}

This section is dedicated to conservative problems, and the inherited numerical approach, namely the finite volume method. Here we focus on the basic conservative equation in the form of

$$
\frac{\partial}{\partial t} u+\frac{\partial}{\partial x} f(u)=0, \quad \text { for } x \in \mathbb{R}, t>0 .
$$

By writing $b(u)=f^{\prime}(u)$, equation (5.0.1) can be written as

$$
\frac{\partial u}{\partial t}+b(u) \frac{\partial u}{\partial x}=0, \quad \text { for } x \in \mathbb{R}, t>0
$$

When $f(u)$ is a quadratic function of $u$, the equation (5.0.2) is a nonlinear partial differential equation. In this chapter we focus on two types of $f(u)$; convex and concave quadratic functions. If $f(u)=\frac{1}{2} u^{2}$ (a convex function), then (5.0.2) becomes the following Burgers' equation

$$
u_{t}+u u_{x}=0
$$

If $f(u)=u(1-u)$ (a concave function), equation (5.0.2) reduces to

$$
u_{t}+(1-2 u) u_{x}=0
$$

which is known as the kinematic equation for traffic flow. In this chapter, analytical solution as well as the numerical method of the Burgers' and traffic flow equations will be discussed 


\subsection{Riemann problem}

Riemann problem is the conservation equation (5.0.2) subject to a piecewise constant initial condition:

$$
u(x, 0)= \begin{cases}u_{\ell}, & \text { if } x<0, \\ u_{r}, & \text { if } x>0 .\end{cases}
$$

For equation (5.0.2) with initial condition (5.1.1), on the half domain $x>0$, $t>0$, the characteristics are straight lines with gradient $b\left(u_{r}\right)$. Whereas on the other half $x<0, t>0$, the characteristics are straight lines with gradient $b\left(u_{\ell}\right)$. Based on different combination of $b\left(u_{\ell}\right)$ and $b\left(u_{r}\right)$, solution of the Riemann problem will be discussed.

But first observe the following; if $u(x, t)$ is a solution, so is $u(a x, a t)$, for an arbitrary $a>0$ (please check!). This implies that solution of (5.0.1) is a similarity solution of the form $u(x, t)=\hat{u}(x / t)$. Further, solutions of $(5.0 .1,5.1 .1)$ are propagated along the characteristic $x / t=b(u)$, with $b(u)=f^{\prime}(u)$ represents the signal velocity. Adopting the initial condition (5.1.1), we can distinguish two cases: $b\left(u_{\ell}\right)>b\left(u_{r}\right)$ and $b\left(u_{\ell}\right)<b\left(u_{r}\right)$, to be discussed below.

Case 1: $b\left(u_{\ell}\right)>b\left(u_{r}\right)$.

The characteristics emanating from $x<0$ have a smaller slope than the slope of the characteristics coming from $x>0$. As a consequence, there is an area in which the characteristics intersect, which would lead to multi valued solutions. The piecewise constant solution of the Riemann problem $(5.0 .1,5.1 .1)$ is given by

$$
u(x, t)= \begin{cases}u_{\ell}, & \text { if } x / t<s, \\ u_{r}, & \text { if } x / t>s,\end{cases}
$$

where $s$ is defined by the Rangkine Hugoniot condition

$$
s=\frac{f\left(u_{r}\right)-f\left(u_{\ell}\right)}{u_{r}-u_{\ell}} .
$$

Solution of (5.1.2) is known as the shock wave, with $s$ the shock speed. This solution contains discontinuity, and therefore it is considered as a weak solution. A typical shock wave solution and the corresponding characteristics are shown in Figure 5.1.1. In Theorem 5.1.1 we will show that the shock speed formula (5.1.3) is determined such that (5.1.2) is a solution of the integral form of (5.0.1). 

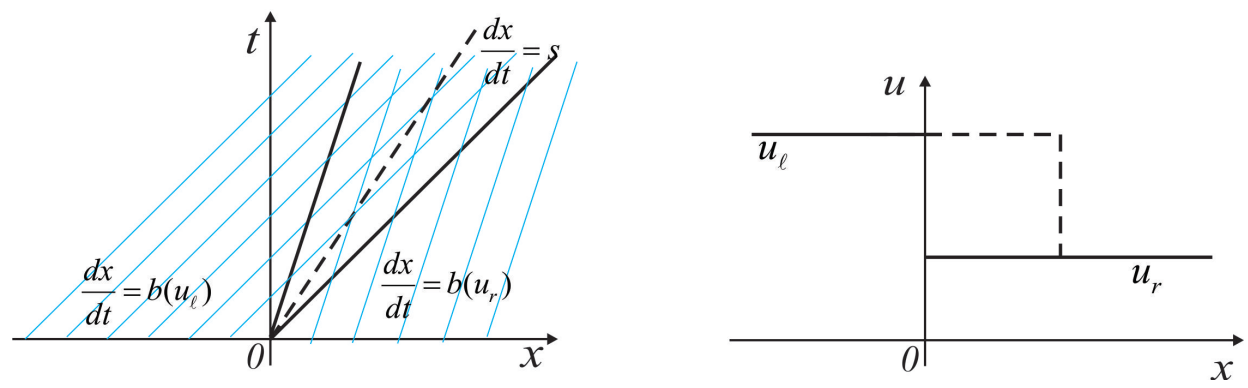

Figure 5.1.1: Intersecting characteristics and the shock wave.
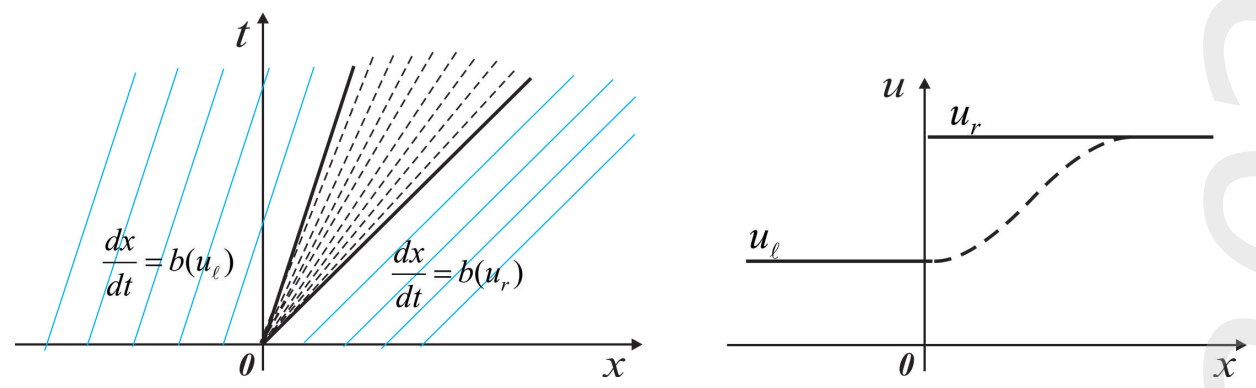

Figure 5.1.2: Separating characteristics and the rarefaction wave.

Case 2: $b\left(u_{\ell}\right)<b\left(u_{r}\right)$.

In this case the characteristic emanating from $x<0$ have a larger slope than those emanating from $x>0$, so we have separating characteristic, see Figure 5.1.2 (left). In this case we do not expect a discontinuous solution. Instead, a solution of (5.0.2) is given by a continuous function

$$
u(x, t)= \begin{cases}u_{\ell}, & \text { if } x / t<b\left(u_{\ell}\right) \\ w(x / t), & \text { if } b\left(u_{\ell}\right)<x / t<b\left(u_{r}\right) \\ u_{r}, & \text { if } x / t>b\left(u_{r}\right) .\end{cases}
$$

where $w(\eta)$ should satisfy $b(w(\eta))=\eta$. This last equation is obtained by requiring $w(x / t)$ to satisfy (5.0.1). This solution is called a rarefaction wave, and its typical profile is depicted in Figure (5.1.2) (right). Despite the fact that the initial condition (5.1.1) is discontinuous, the rarefaction wave (5.1.4) is continuous (please check!).

Teorema 5.1.1. Discontinuity of the shock wave solution (5.1.2) moves with velocity (5.1.3) 


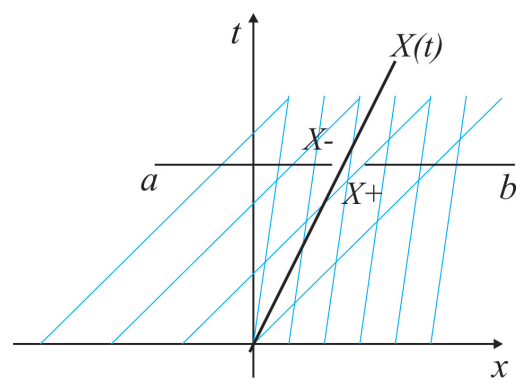

Figure 5.1.3: A shock formed between crossing characteristics.

Proof: Shock wave $(5.1 .2)$ is a solution of $(5.0 .1,5.1 .1)$ that contains discontinuity. As a discontinuous solution, it certainly does not satisfy the differential equation (5.0.1) in the normal sense. Here, we will show that is satisfies the integral form of (5.0.1). Further, the Rangkine-Hugoniot formula (5.1.3) is derived from the integral form of (5.0.1). Suppose $x=X(t)$ denotes the discontinuity position. Let us take two arbitrary points $x=a$ and $x=b$ in such a way that $a<X(t)<b$. The integral form of (5.0.1) must still hold even across a shock. The integral form of (5.0.1) over $x=a$ and $x=b$ reads

$$
\frac{\partial}{\partial t} \int_{a}^{b} u d x+f(b, t)-f(a, t)=0 .
$$

From the above relation we can get the following

$$
\begin{gathered}
\frac{\partial}{\partial t} \int_{a}^{X^{-}} u d x+\frac{\partial}{\partial t} \int_{X^{+}}^{b} u d x+f(b, t)-f\left(X^{+}, t\right)+f\left(X^{-}, t\right)-f(a, t)=0 \\
\int_{a}^{X^{-}} \frac{\partial u}{\partial t} d x+\dot{X} u\left(X^{-}\right)+\int_{X^{+}}^{b} \frac{\partial u}{\partial t} d x-\dot{X} u\left(X^{+}\right)+ \\
\int_{a}^{X^{-}} \frac{\partial f}{\partial x} d x+[f]_{X^{+}}^{X^{+}}+\int_{X^{+}}^{b} \frac{\partial f}{\partial x} d x=0 \\
\int_{a}^{X^{-}} \frac{\partial u}{\partial t}+\frac{\partial f}{\partial x} d x+\int_{X^{+}}^{b} \frac{\partial u}{\partial t}+\frac{\partial f}{\partial x} d x-\dot{X}[u]_{X^{-}}^{X^{+}}+[f]_{X^{-}}^{X^{+}}=0
\end{gathered}
$$

Thus the shock move according to

$$
\dot{X}=\frac{[f]}{[u]} .
$$




\section{Exercise 5.1.2.}

1. Burgers' equation is equation (5.0.1) with the flux $f(u)=\frac{1}{2} u^{2}$, written explicitly as follows

$$
\frac{\partial u}{\partial t}+\frac{\partial}{\partial x}\left(\frac{1}{2} u^{2}\right)=0
$$

subject to the piecewise constant initial condition

$$
u(x, 0)= \begin{cases}u_{\ell}, & \text { if } x<0, \\ u_{r}, & \text { if } x>0 .\end{cases}
$$

In this case $b(u)=f^{\prime}(u)=u$, therefore two type of solutions are distinguish by the relation between $b\left(u_{\ell}\right)=u_{\ell}$ and $b\left(u_{r}\right)=u_{r}$.

If $u_{\ell}>u_{r}$, we have an area in which characteristic intersects, which would lead to a multivalued solution. In this case we have a discontinuous solution. We can easily verify that the solution is

$$
u(x, t)= \begin{cases}u_{\ell}, & \text { if } x / t<s, \\ u_{r}, & \text { if } x / t>s,\end{cases}
$$

where $s$ is defined as

$$
s=\frac{f\left(u_{\ell}\right)-f\left(u_{r}\right)}{u_{\ell}-u_{r}} .
$$

Solution (5.2.8) is called shock wave. Discontinuity of the shock wave propagates with speed $s$ that follows from the Rangkine-Hugoniot condition (5.1.6). Complete derivation can be found ini [10] Subsection 12.2.2.

If $u_{\ell}<u_{r}$, we have a separating characteristic, and we expect to have a continuous solution. The solution is given by

$$
u(x, t)= \begin{cases}u_{\ell}, & \text { if } x / t<b\left(u_{\ell}\right), \\ w(x / t), & \text { if } b\left(u_{\ell}\right)<x / t<b\left(u_{r}\right) \\ u_{r}, & \text { if } x / t>b\left(u_{r}\right),\end{cases}
$$

where $w(\eta)$ follows from $b(w(\eta))=\eta$. Show that for Burgers' equation $w$ is an identity function, thus the rarefaction wave solution reads

$$
u(x, t)= \begin{cases}u_{\ell}, & \text { if } x / t<u_{\ell} \\ x / t, & \text { if } u_{\ell}<x / t<u_{r} \\ u_{r}, & \text { if } x / t>u_{r}\end{cases}
$$


2. The kinematic equation for traffic flow is equation (5.0.1) with the chosen flux function. Commonly used function is the Greenshield flux, in which after normalization $f(u)=u(1-u)$. Similarly, there are two type of solutions of

$$
\frac{\partial u}{\partial t}+\frac{\partial}{\partial x}(u(1-u))=0
$$

subject to the initial condition

$$
u(x, 0)= \begin{cases}u_{\ell}, & \text { if } x<0, \\ u_{r}, & \text { if } x>0 .\end{cases}
$$

In this case $b(u)=f^{\prime}(u)=1-2 u$, therefore two type of solutions are distinguish by the relation between $b\left(u_{\ell}\right)=1-2 u_{\ell}$ and $b\left(u_{r}\right)=1-2 u_{r}$.

If $u_{\ell}<u_{r}$, then $b\left(u_{\ell}\right)>b\left(u_{r}\right)$, and the solution is a shock wave. Formulate this shock wave, and determine the shock speed. If $u_{\ell}>u_{r}$, then $b\left(u_{\ell}\right)<b\left(u_{r}\right)$, and the solution is a rarefaction wave. Formulate this rarefaction wave solution.

\subsection{Finite volume method}

The numerical finite volume method is closely related to the finite difference method. Sometimes, a finite volume scheme is misinterpreted as a finite difference approximation to a differential equation. However, finite volume methods are different, they are derived on the basis of the integral form of the conservation law, a starting point that turns out to have many advantages. Whereas in finite difference methods all derivatives appear in the equation, each is approximated by a ratio of differences.

Consider the standard conservation equation with a flux function $f(u)$ and the initial condition below

$$
\begin{gathered}
\frac{\partial u}{\partial t}+\frac{\partial f(u)}{\partial x}=0, \quad x \in \mathbb{R}, t>0, \\
u(x, 0)=v(x), \quad x \in \mathbb{R} .
\end{gathered}
$$

Generally $f(u)$ is a nonlinear function, and nonlinear problems are essentially more difficult than linear ones. In particular, nonlinear equations allow a smooth initial condition to developed into a discontinuous solution. This is of course put extra requirements on the numerical scheme. 


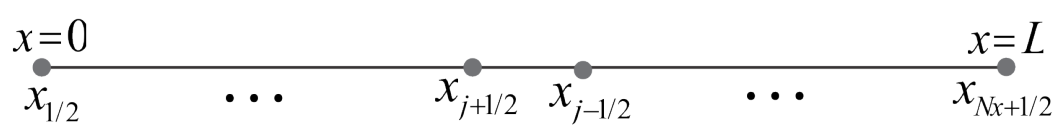

Figure 5.2.1: The spatial domain with the staggered grid points.

Here, we will discuss the finite volume method starting from the simplest case. On the computational domain $[0, L]$ which is divided into $N_{x}$ cells of homogeneous length $\Delta x$, a staggered partition points are

$$
x_{1 / 2}=0, x_{3 / 2}=\Delta x, \cdots, x_{j+1 / 2}=j \Delta x, \cdots, x_{N_{x}+1 / 2}=N_{x} \Delta x=L,
$$

see Figure 5.2.2. We assume $u\left(x_{j}, t^{n}\right)$ denotes the averaged value of $u$ in cell $V_{j}=\left[x_{j-\frac{1}{2}}, x_{j+\frac{1}{2}}\right]$, see Fig 5.2.2. Within time interval $\Delta t$, this value changes according to the net flux from the left and right boundaries of cell $V_{j}$ according to

$$
u\left(x_{j}, t_{n+1}\right) \Delta x=u\left(x_{j}, t_{n}\right) \Delta x+\Delta t\left(f\left(x_{j-\frac{1}{2}}, t_{n}\right)-f\left(x_{j+\frac{1}{2}}, t_{n}\right)\right) .
$$

Equation (5.2.4) is exactly the conservative principle in discrete form of the quantity $u$ on cell $V_{j}$. Also, equation (5.2.4) can be interpreted as the integral form of the conservation law (5.2.1) on cell $V_{j}$. For further discussion, we use the following notations $u_{j}^{n}=u\left(x_{j}, t_{n}\right)$, and $f_{j}^{n}=f\left(x_{j-\frac{1}{2}}, t_{n}\right)$, for all $x_{j-\frac{1}{2}}$ and $t^{n}$ in the computational domain. What to do next is formulating a good approximation for the flux $f_{j+\frac{1}{2}}^{n}$. This is in fact the most important ingredient of a finite volume method (5.2.5).

The first attempt to approximate $f_{j+\frac{1}{2}}^{n}$ is to use an average

$$
f_{j+\frac{1}{2}}^{n}=\frac{1}{2}\left(f\left(u_{j}^{n}\right)+f\left(u_{j+1}^{n}\right)\right) .
$$

By taking this approximation, the finite volume method (5.2.4) becomes

$$
u_{j}^{n+1}=u_{j}^{n}-\frac{\Delta t}{2 \Delta x}\left(f\left(u_{j+1}^{n}\right)-f\left(u_{j-1}^{n}\right)\right) .
$$

Unfortunately, this method is generally unstable for hyperbolic problems, and therefore it cannot be used.

In the following examples, we will show how to choose a flux function that suits the differential equation that we consider. In these simple cases, the finite volume method reduces to the finite difference methods. 


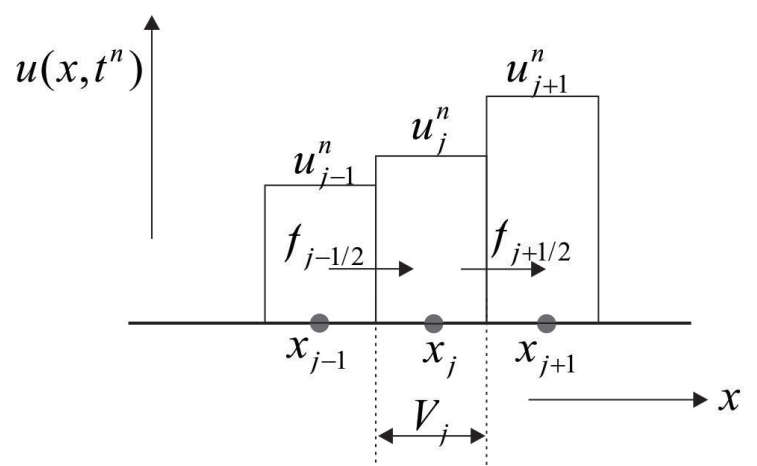

Figure 5.2.2: Sketch of the spatial domain in the finite volume method, and illustration of the piecewise constant numerical solution at time level $t^{n}$.

1. If the flux $f(u)=-\kappa u_{x}$, equation (5.2.1) reduces to diffusion equation $u_{t}=\kappa u_{x x}$. For the approximation, we take an average

$$
f_{j+\frac{1}{2}}^{n}=-\kappa \frac{u_{j+1}^{n}-u_{j}^{n}}{\Delta x},
$$

the discrete equation (5.2.4) reduces to

$$
u_{j}^{n+1}=u_{j}^{n}+\kappa \frac{\Delta t}{\Delta x^{2}}\left(u_{j+1}^{n}-2 u_{j}^{n}+u_{j-1}^{n}\right),
$$

which is exactly the finite difference approximation (3.3.4).

2. If the flux $f(u)=d u$, equation (5.2.1) reduces to convection equation $u_{t}+d u_{x}=0$. Here we take the upwind approximation:

- If $d>0, f_{j+\frac{1}{2}}^{n}=d u_{j}^{n}$, and the discrete equation (5.2.4) reduces to

$$
u_{j}^{n+1}=u_{j}^{n}-d \frac{\Delta t}{\Delta x}\left(u_{j}^{n}-u_{j-1}^{n}\right)
$$

which is exactly the FTBS scheme of the convection equation.

- If $d<0, f_{j+\frac{1}{2}}^{n}=d u_{j+1}^{n}$, and the discrete equation (5.2.4) reduces to

$$
u_{j}^{n+1}=u_{j}^{n}-d \frac{\Delta t}{\Delta x}\left(u_{j}^{n}-u_{j-1}^{n}\right)
$$

which is exactly the FTFS scheme of the convection equation. 


\subsubsection{Godunov method}

Consider the Burgers' equation which is the conservative equation with $f(u)=\frac{1}{2} u^{2}$, written explicitly as

$$
\frac{\partial u}{\partial t}+\frac{\partial}{\partial x}\left(\frac{1}{2} u^{2}\right)=0, \quad x \in \mathbb{R}, t>0
$$

As illustrated in Figure 5.2.2, for each cell $V_{j}=\left[x_{j-1 / 2}, x_{j+1 / 2}\right]$ in the computational domain, an approximation of $f_{j+\frac{1}{2}}^{n}$ is needed. In Godunov method, the approximation of $f_{j+\frac{1}{2}}^{n}$ is based on the analytical solution.

Here, we first discuss the analytical solution of (5.2.6) subjects to the initial condition

$$
u\left(x, t^{n}\right)= \begin{cases}u_{j}^{n}, & x<x_{j+1 / 2} \\ u_{j+1}^{n}, & x>x_{j+1 / 2} .\end{cases}
$$

- If $u_{j}^{n}>u_{j+1}^{n}$, the solution is a shock wave propagating with speed

$$
s_{j}^{n}=\frac{1}{2}\left(u_{j}^{n}+u_{j+1}^{n}\right) .
$$

Let us denote

$$
\eta=\frac{x-x_{j+\frac{1}{2}}}{t-t^{n}}
$$

Then, the shock wave solution is given by

$$
u_{R}\left(\eta ; u_{j}^{n}, u_{j+1}^{n}\right)= \begin{cases}u_{j}^{n}, & \text { if } \eta<s_{j}^{n} \\ u_{j+1}^{n}, & \text { if } \eta>s_{j}^{n} .\end{cases}
$$

- If $u_{j}^{n}<u_{j+1}^{n}$, the solution is a rarefaction wave as given by

$$
u_{R}\left(\eta ; u_{j}^{n}, u_{j+1}^{n}\right)= \begin{cases}u_{j}^{n}, & \text { if } \eta<u_{j}^{n} \\ \eta, & \text { if } u_{j}^{n}<\eta<u_{j+1}^{n} \\ u_{j+1}^{n}, & \text { if } \eta>u_{j+1}^{n} .\end{cases}
$$

By taking the numerical flux $f_{x_{j}+\frac{1}{2}}=\frac{1}{2} u_{R}^{2}\left(\eta=0 ; u_{j}^{n}, u_{j+1}^{n}\right)$, we obtained the following 
- If $u_{j}^{n}>u_{j+1}^{n}$, then

$$
f_{j+\frac{1}{2}}^{n}= \begin{cases}\frac{1}{2}\left(u_{j}^{n}\right)^{2}, & \text { if } s_{j}^{n}>0 \\ \frac{1}{2}\left(u_{j+1}^{n}\right)^{2}, & \text { if } s_{j}^{n}<0\end{cases}
$$

- If $u_{j}^{n}<u_{j+1}^{n}$

$$
f_{j+\frac{1}{2}}^{n}= \begin{cases}\frac{1}{2}\left(u_{j}^{n}\right)^{2}, & \text { if } u_{j}^{n}>0 \\ 0, & \text { if } u_{j}^{n}<0<u_{j+1}^{n} \\ \frac{1}{2}\left(u_{j+1}^{n}\right)^{2}, & \text { if } u_{j+1}^{n}<0 .\end{cases}
$$

We resume here that the Godunov method for the Burgers' equation is (5.2.4) with the flux function is given by (5.2.10) or (5.2.11).
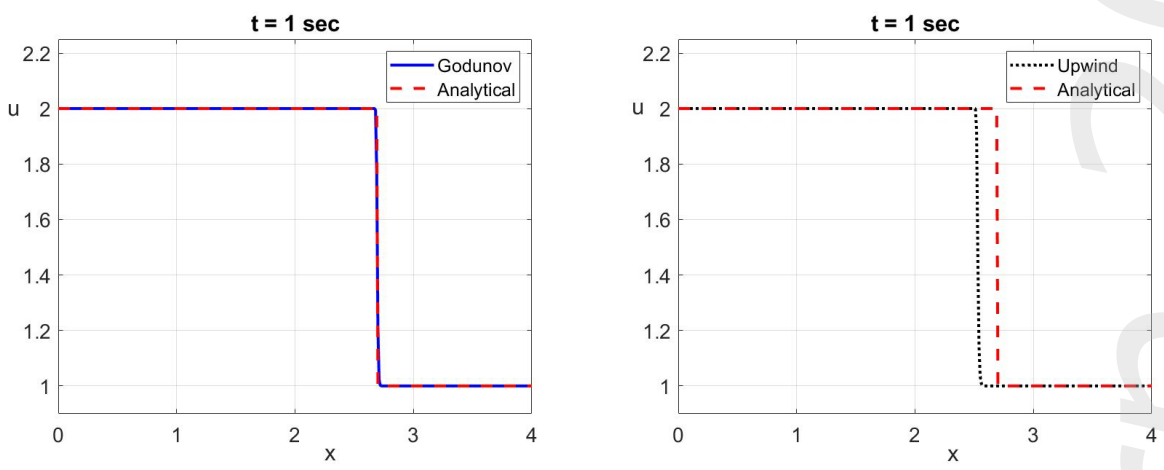

Figure 5.2.3: Shock wave solutions at time $t=1$ calculated using Godunov method (left) and Upwind method (right), in comparison with the analytical solution.

\section{Exercise 5.2.1.}

1. Solve the Burgers' equation subject to the initial condition

$$
u(x, 0)= \begin{cases}2, & \text { if } x<0 \\ 1, & \text { if } x>0\end{cases}
$$

For this initial condition, it holds $u_{j}^{n}>u_{j+1}^{n}$, for all $j, n$ in the computational region, hence, an approximation for the flux function read 
as $f_{j+\frac{1}{2}}=\frac{1}{2} u_{R}^{2}\left(\eta=0 ; u_{j}^{n}, u_{j+1}^{n}\right)=\frac{1}{2}\left(u_{j}^{n}\right)^{2}$. In this case the scheme reduces to

$$
u_{j}^{n+1}=u_{j}^{n}-\frac{\Delta t}{\Delta x}\left(\frac{1}{2}\left(u_{j}^{n}\right)^{2}-\frac{1}{2}\left(u_{j-1}^{n}\right)^{2}\right)
$$

Implement this Godunov method, and write a numerical code to simulate the propagation of a shock wave solution. Use $\Delta x=2.5 \times$ $10^{-2}, \Delta t=1.25 \times 10^{-2}$ and plot numerical solution together with the analytical solution at time $t=1$. Note that the analytical solution will be a shock wave, with discontinuity that propagates with speed $s=1.5$.

2. Alternatively, Burgers' equation can be written as

$$
u_{t}+u u_{x}=0 .
$$

Implementation of the upwind scheme, and since $u>0$ we obtain the following scheme

$$
u_{j}^{n+1}=u_{j}^{n}-\frac{\Delta t}{\Delta x} u_{j}^{n}\left(u_{j}^{n}-u_{j-1}^{n}\right) .
$$

Write a numerical code to simulate the propagation of a shock wave solution. Use $\Delta x=0.025, \Delta t=0.0125$ and plot numerical solution together with the analytical solution at time $t=1$. It is shown in (5.2.3) that the upwind method cannot capture the shock speed correctly.

3. In Godunov method for Burgers' equation, flux function is approximated according to $f_{j+\frac{1}{2}}=\frac{1}{2} u_{R}^{2}\left(\eta=0 ; u_{j}^{n}, u_{j+1}^{n}\right)$. Find the explicit approximation for $f_{j+\frac{1}{2}}$ in the case of

(a) $0<u_{j}^{n}<u_{j+1}^{n}$,

(b) $u_{j+1}^{n}<u_{j}^{n}<0$,

(c) $u_{j}^{n}<u_{j+1}^{n}<0$,

(d) $u_{j+1}^{n}<0<u_{j}^{n}$ with $s_{j}^{n}<0$,

(e) $u_{j}^{n}<0<u_{j+1}^{n}$.

Implement your formula above and simulate the Riemann problem.

4. Implement the Godunov method to solve the Burgers' equation (5.2.6) with an initial condition

$$
u(x, 0)= \begin{cases}u_{\ell}, & \text { if } x<0 \\ u_{r}, & \text { if } x>0 .\end{cases}
$$


for arbitrary $u_{\ell}, u_{r}$. Carefully formulate the flux function using $f_{x_{j}+\frac{1}{2}}=$ $\frac{1}{2} u_{R}^{2}\left(\eta ; u_{j}^{n}, u_{j+1}^{n}\right)$, with $u_{R}$ denotes the analytical solution. Your code should be able to simulate various conditions that lead to a shock wave as well as a rarefaction wave as predicted analytically.

5. Consider $u_{t}+u^{2} u_{x}=0$. Simulate a shock wave solution emanated from

$$
u(x, 0)= \begin{cases}2, & \text { if } x<0 \\ 1, & \text { if } x>0\end{cases}
$$

Simulate a rarefaction wave solution emanated from

$$
u(x, 0)= \begin{cases}1, & \text { if } x<0 \\ 2, & \text { if } x>0\end{cases}
$$

Clearly the Godunov method (5.2.12) is much better compare with the upwind scheme (5.2.14). The Godunov scheme can capture the speed of the shock wave correctly. This is an important issue, and a characteristic features of a conservative scheme. Whereas, the scheme (5.2.14) even tough it is stable, it fails to capture the correct speed.

\subsection{The kinematic LWR model for traffic flow}

Imagine a stream of cars traveling along a one-lane highway. Obviously, it is a discrete system with cars being the molecules. However this is merely our scale of perception. If we were high above the road then we won't be able to see each individual cars, we would only be able to distinguish places where there were a lot of cars and places where there were few. On this macroscopic scale, we can as well consider traffic flow as a continuum with dependent variable traffic density $n(\mathrm{cars} / \mathrm{km})$. Two others variables are: the averaged velocity of cars $v(\mathrm{~km} /$ hour$)$, and traffic flux $f$ (cars/hour), which is the number of cars passing through a certain position, per unit of time. As a rule of thumb, traffic flux equals to traffic density times velocity, $f=n v$.

Consider a one lane traffic moving in one direction, say the $x$-direction. Let $n(x, t)($ cars $/ \mathrm{km})$ represents traffic density as a function of the spatial variable $x$ and time $t$. Consider a road segment $[x, x+\Delta x]$ illustrated in Figure 5.3.2, within time interval $\Delta t$, total number of cars in that segment changes from $n(x, t) \Delta x$ to $n(x, t+\Delta t) \Delta x$. This change is caused by cars that enter from the boundary $x$ with flux $f(x, t) \Delta t$, and exit from $x+\Delta x$ 


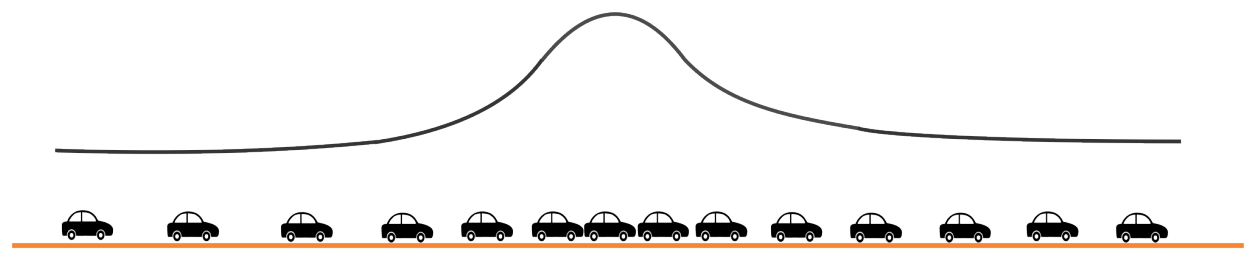

Figure 5.3.1: A pulse of traffic density $n(x, t)($ cars $/ \mathrm{km})$ in the macroscopic approach.

with flux $f(x+\Delta x, t) \Delta t$. In addition, there are cars that enter or exit with rate $\beta$ (cars $/ \mathrm{km}$.hour). Combining all, we get the following in-out relation:

$$
n(x, t+\Delta t) \Delta x-n(x, t) \Delta x=f(x, t) \Delta t-f(x+\Delta x, t) \Delta t+\beta \Delta x \Delta t .
$$

Divide both sides with $\Delta x \Delta t$, and take the limit $\Delta x \rightarrow 0$ and $\Delta t \rightarrow 0$, we will get

$$
\partial_{t} n+\partial_{x} f=\beta .
$$

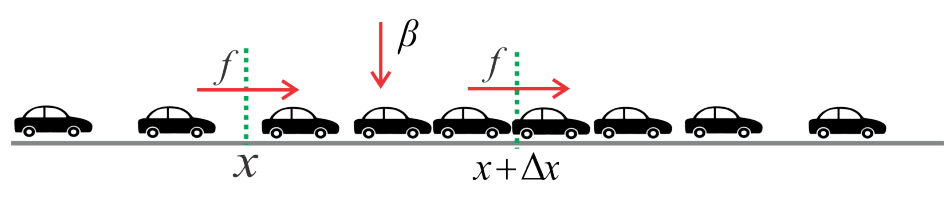

Figure 5.3.2: Illustration of the control volume $[x, x+\Delta x]$ with the in-out relation.

When there is no exits-entrances or $\beta=0$, the conservative principle of traffic flow is described by the homogeneous kinetic LWR equation below

$$
\partial_{t} n+\partial_{x} f(n)=0
$$

The abbreviation LWR stands from Lighthill, Whitham and Richard, who proposed this model $[8,9,11]$. To solve the equation above, one needs an explicit formula for the flux function $f(n)$. Here we present several options to model the flux function $f(n)$.

Figure 5.3.3 illustrates an image captured by a video camera installed above a highway; the left figure is data of speed versus density, whereas the 

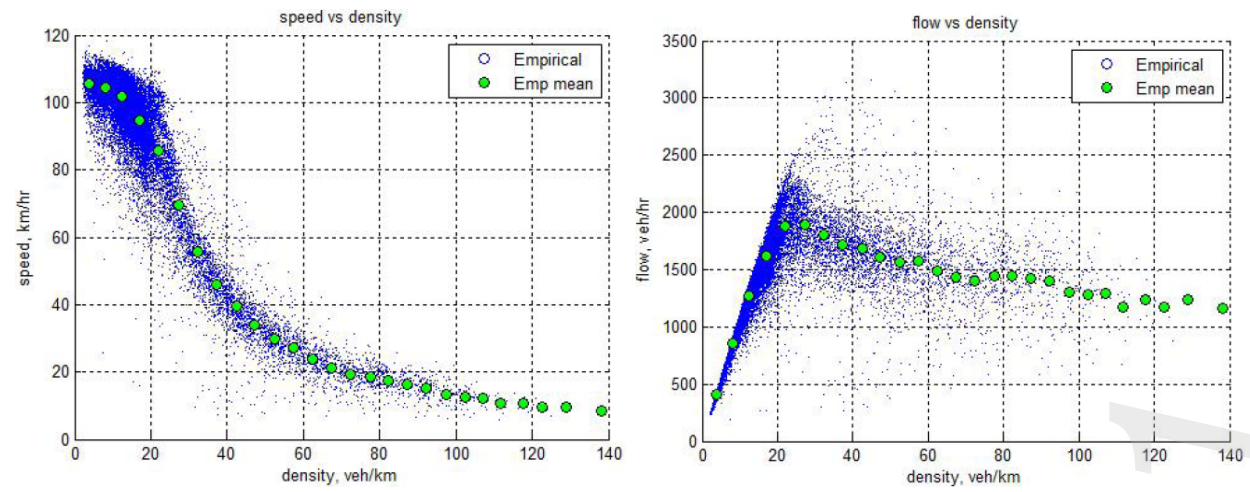

Figure 5.3.3: Observed velocity-density-flux relationships (data source: NaviGAtor).

right figure contains data of traffic flux versus density. Actually, these data points depicts an equilibrium or steady-state relationship between velocity, density and flux. It is shown in Figure 5.3.3 (left), when road is relatively empty (traffic density is low) the averaged velocity is high, whereas on busy road (high traffic density) velocity is low. Clearly, the averaged velocity depends on traffic density.
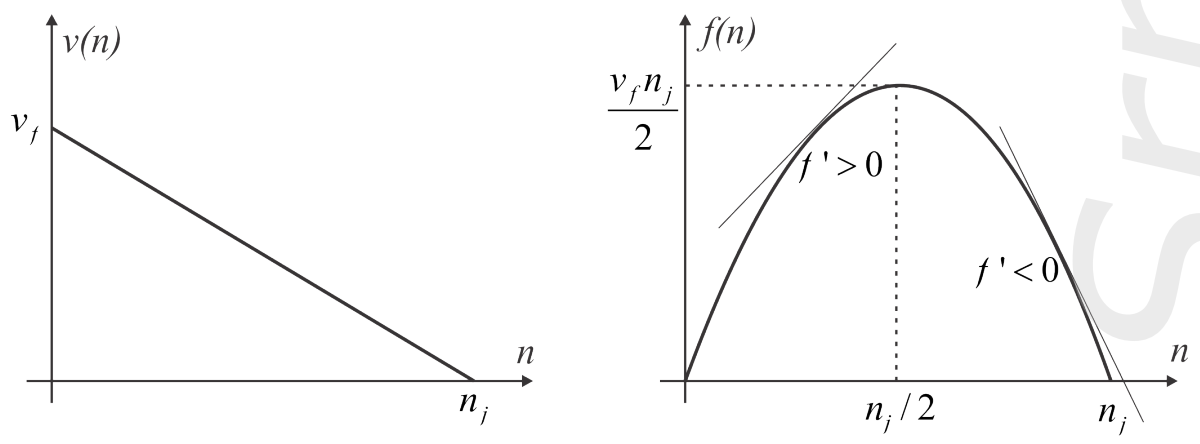

Figure 5.3.4: The Greenshield velocity $v(n)$ (left), and the corresponding flux $f(n)=n v(n)$ (right).

For the equilibrium state, there are several models of velocity as a function of density. The simplest one is Greenshield, which assumes the averaged 
velocity depends of traffic density $n$ as a linear function

$$
v(n)=v_{f}\left(1-\frac{n}{n_{j}}\right),
$$

where $v_{f}(\mathrm{~km} /$ hour $)$ denotes the full speed velocity of cars on empty roads, and $n_{j}(\mathrm{cars} / \mathrm{km})$ denotes the maximum capacity of a one lane traffic. Next, traffic flux and traffic density are related as $f=n v$, with $v$ denotes the averaged velocity of cars. Adopting Greenshield velocity, traffic flux is then

$$
f(n)=n v(n)=n v_{f}\left(1-\frac{n}{n_{j}}\right) .
$$

In Figure 5.3.4, the equilibrium Greenshield model of velocity and flux are plotted as functions of density. We shall note that by taking the Greenshield model, different behavior of drivers, relaxed or rushed, is ignored. Instead, the vehicle velocity $v$ is assumed to depends only on the surrounding traffic density $n$. Although there appears a considerable deviation, the Greenshield model has captured two main characteristic of traffic flow: the flux is zero when traffic density is zero $n=0$ or maximum $n=n_{j}$, whereas the flux is maximum when $n \approx n_{j} / 2$.

The advantage of adopting Greenshield model is because the analytical solution is available. We can use this Greenshield analytical solution to test our numerical scheme. For real applications and more relevant analysis, there are better suited models. Several models as proposed in literature [3] are Greenberg, Underwood, and Pipes-Munjal, with velocity functions as follows

$$
\begin{array}{cl}
\text { Greenshield } \quad v(n)=v_{f}\left(1-\frac{n}{n_{j}}\right), \\
\text { Greenberg } \quad v(n)=v_{m} \ln \left(\frac{n_{j}}{n}\right), \\
\text { Underwood } v(n)=v_{f} \exp \left(-\frac{n}{n_{m}}\right), \\
\text { Pipes-Munjal } \quad v(n)=v_{f}\left(1-\left(\frac{n}{n_{j}}\right)^{1 / 2}\right) .
\end{array}
$$

In the above models, the same parameters free flow velocity $v_{f}$ and jam density $n_{j}$ are used, whereas parameters $v_{m}$ and $n_{m}$ denote velocity and density that correspond to maximum flux, respectively. Different function of velocity $v(n)$ directly leads to a different flux model $f(n)=n v(n)$. 


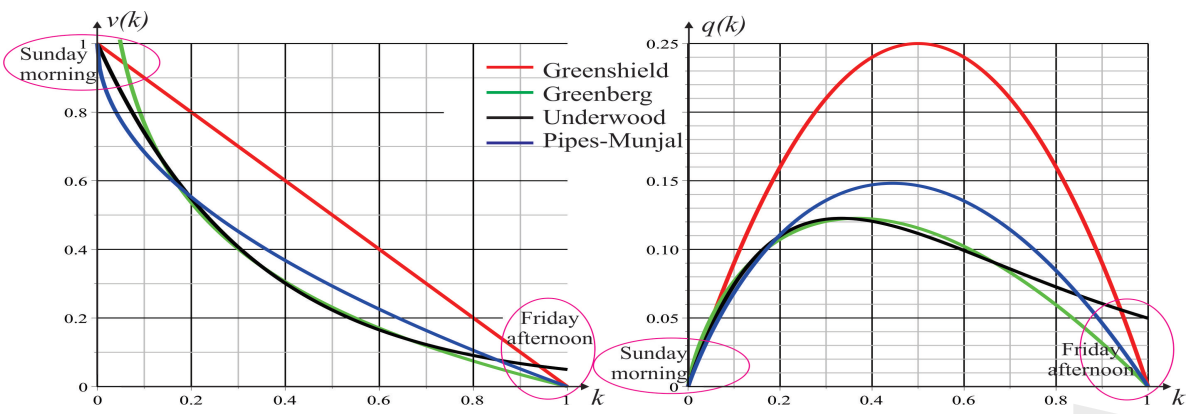

Figure 5.3.5: Several traffic flow models (left) velocity density relationship, (right) flux density relationship.

\section{Exercise 5.3.1.}

1. Given the car flux density relation $f(n)=n(1-n / 150)(1-n / 300)$ cars per minute, where $n$ is measured in cars per $\mathrm{km}$, and $0 \leq n \leq$ 150 .

(a) Sketch $f(n)$, the velocity $v(n)$, and the wave speed $b(n)=f^{\prime}(n)$. Indicate the values and the corresponding points on the graphs of the maximum density of cars, the maximum flux of cars, the velocity of cars at the maximum flux, and the maximum velocity of cars.

(b) Sketch a characteristic correspond to the condition 'when traffic light turns green'

$$
n(x, 0)= \begin{cases}150, & \text { if } x<0 \\ 0, & \text { if } x>0\end{cases}
$$

Use a scale to cover the range $\pm 3 \mathrm{~km}$ and $0-3$ minutes.

(c) Graph the solution $n(x, t)$ at times $t=1,2$ and 3 minutes.

2. The initial value problem $\partial_{t} n+b(n) \partial_{x} n=0$ such that $n(x, 0)=$ $n_{0}(x)$ has the solution $n=n_{0}(s)$ on characteristic $x=s+b_{0}(s) t$ where $b_{0}(s)=b\left(n_{0}(s)\right)$. Show that indeed $n=n_{0}(s(x, t))$ satisfy the governing differential equation. (Hint: regard $x=s+b_{0}(s) t$ as an implicit equation for the function $s(x, t)$ : differentiate it to find implicit formulae for $\frac{\partial s}{\partial t}$ and $\frac{\partial s}{\partial x}$.)

3. Traffic flow model using Greenshield flux function is

$$
\partial_{t} n+\partial_{x} f(n)=0, \text { with } f(n)=n V_{f}\left(1-\frac{n}{n_{j}}\right) .
$$


Introduce the following non-dimensional variables: $u=n / n_{j}, \hat{x}=$ $x / L, \hat{t}=t / L$, where $L, T$ are typical length and time scales, such that $L / T=v_{f}$, then derive the 'normalized' form of the traffic flow model below

$$
\partial_{\hat{t}} u+\partial_{\hat{x}}(u(1-u))=0 .
$$

4. A more realistic traffic model can be obtained if we take into account driver's intelligence, which is as follows. If they see denser traffic ahead (in which case $\frac{\partial n}{\partial x}>0$ ) then they slow down. Mathematically, this could be described by $v(n)-\frac{\mu}{n} \frac{\partial n}{\partial x}$, with a positive constant $\mu$. How does the driver respond if he sees lighter traffic ahead? With this new velocity formula, show that traffic flow model becomes

$$
\partial_{t} n+\partial_{x}(n v(n))=\mu \partial_{x x} n .
$$

So the use of this new speed formula will give a damping effect in the traffic flow model.

Exercise 5.3.2. Riemann solutions of traffic flow equation

Consider the kinematic LWR model with the Greenshield flux $f(u)=$ $u(1-u)$, after normalization by taking $v_{f}=1, n_{j}=1$. Similar to Burgers' equation, there will be two solution types of the kinematic wave equation

$$
\frac{\partial u}{\partial t}+\frac{\partial}{\partial x}(u(1-u))=0
$$

subject to initial condition

$$
u(x, 0)= \begin{cases}u_{\ell}, & \text { if } x<0 \\ u_{r}, & \text { if } x>0,\end{cases}
$$

In this case $b(u)=f^{\prime}(u)=1-2 u$, therefore two type of solutions are distinguished by the relation between $b\left(u_{\ell}\right)=1-2 u_{\ell}$ and $b\left(u_{r}\right)=1-2 u_{r}$. If $u_{\ell}<u_{r}$, then $b\left(u_{\ell}\right)>b\left(u_{r}\right)$, show that the solution is a shock wave

$$
u(x, t)= \begin{cases}u_{\ell}, & \text { if } x / t<s, \\ u_{r}, & \text { if } x / t>s,\end{cases}
$$

with the shock speed follows from

$$
s=\frac{f\left(u_{r}\right)-f\left(u_{\ell}\right)}{u_{r}-u_{\ell}} .
$$


Further, if $u_{\ell}>u_{r}$, then $b\left(u_{\ell}\right)<b\left(u_{r}\right)$, show that the solution is a rarefaction wave

$$
u(x, t)= \begin{cases}u_{\ell}, & \text { if } x / t<b\left(u_{\ell}\right) \\ \frac{1}{2}\left(1-\frac{x}{t}\right), & \text { if } b\left(u_{\ell}\right)<x / t<b\left(u_{r}\right) \\ u_{r}, & \text { if } x / t>b\left(u_{r}\right) .\end{cases}
$$

\subsection{Shock and rarefaction waves in traffic flow}

In the following, we will discuss the shock wave solution of traffic flow, whose interpretation we often encounter in daily experience. Let us consider a concave traffic flux curve as depicted in Figure 5.4.1 (Top). Consider two situations $A$ and $B$ characterized by its flux, velocity and density: $f_{A}, v_{A}, n_{A}$, and $f_{B}, v_{B}, n_{B}$, respectively. In this example $n_{A}<n_{B}, f_{A}>f_{B}$, and $v_{A}>v_{B}$. These two conditions can be interpreted as traffic situation on highway as depicted in Figure 5.4.1 (Bottom). At the downstream part of the highway, traffic situation is dense (condition $B$ ), whereas on the upstream part, traffic situation is less dense (condition $A$ ). From top view, we observe a shock wave that connects the high density traffic $n_{B}$ to the lower density $n_{A}$. This shock wave front propagates backward with constant velocity which is the gradient $m_{A B}$ of the segment line $A B$

$$
\frac{d x}{d t}=m_{A B}=\frac{f_{B}-f_{A}}{n_{B}-n_{A}} .
$$

Velocity (5.4.1) is known as the Rangkine-Hugoniot formula. Moreover, as depicted in Fig. 5.4.1 (lower left) the shock line passing through the origin determines the position of shock wave front as a function of time. This shock line also separates two different traffic situations: high density $n_{B}$ and low density $n_{A}$. Further, we summarize that a line segment $A B$ with negative gradient, in the flux curve, corresponds to a backwards propagating shock wave. Analogously, a line segment on the flux curve with positive gradient corresponds to a forward propagating shock wave. Detailed discussion about this can be obtained in $[10,3]$.

The second example is when a traffic light turns green, this situation can be well described by considering a Riemann problem with $n_{A}=n_{j}$, and $n_{B}=0$. In this case a rarefaction wave will emanate, typical situation is depicted in Figure 5.4.2. Later, in Exercise 5.5.1 you will be able to produce this rarefaction wave; analytically and numerically by implementing the finite volume method. 


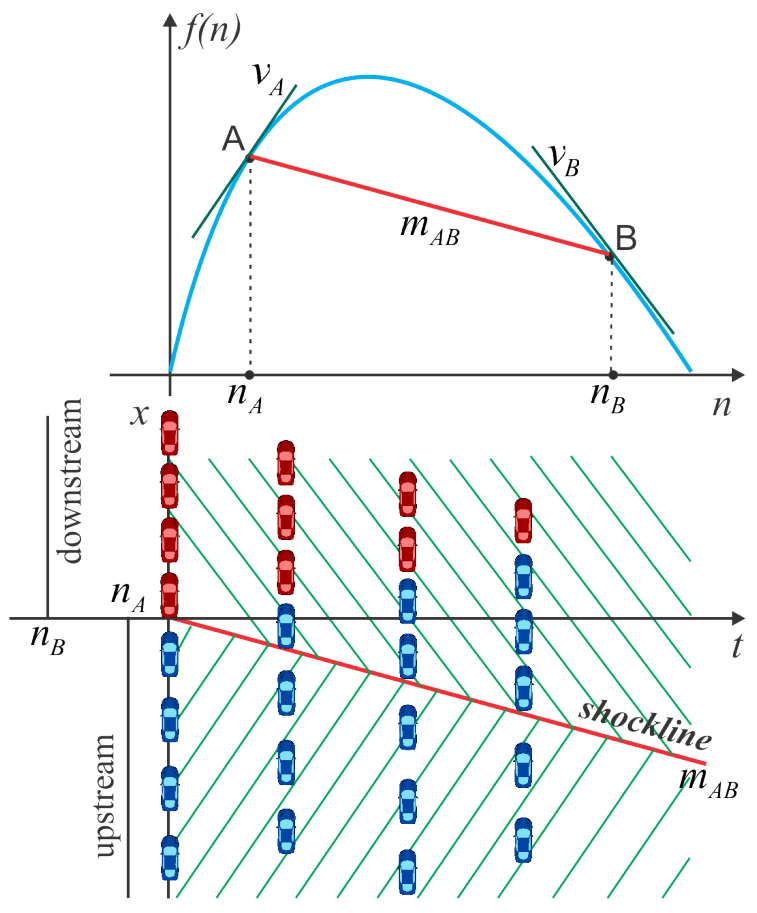

Figure 5.4.1: (Top) The flux curve with two traffic situations $A$ and $B$, connected with a segment line with gradient $m_{A B}$. (Bottom) Top view of a dense traffic condition $B$ propagating backward to the less dense traffic condition $A$.

By considering the two examples above, we assert that any traffic situation can be represented as a point on the flux-density curve. Whereas, transition between traffic situations can be well represented by a line segment connecting two conditions in the flux curve. This graphical method is direct and it holds for any traffic flux model in the form of a concave function. Therefore, it can be employed quite easily by traffic engineers for analyzing and managing traffic regulation.

\subsection{Finite volume method for the kinematic LWR model}

In this section we will discuss the finite volume method for solving the kinematic LWR model with Greenshield flux function.

$$
\begin{aligned}
& \partial_{t} n+\partial_{x} f(n)=0 \\
& f(n)=n v_{f}\left(1-\frac{n}{n_{j}}\right)
\end{aligned}
$$




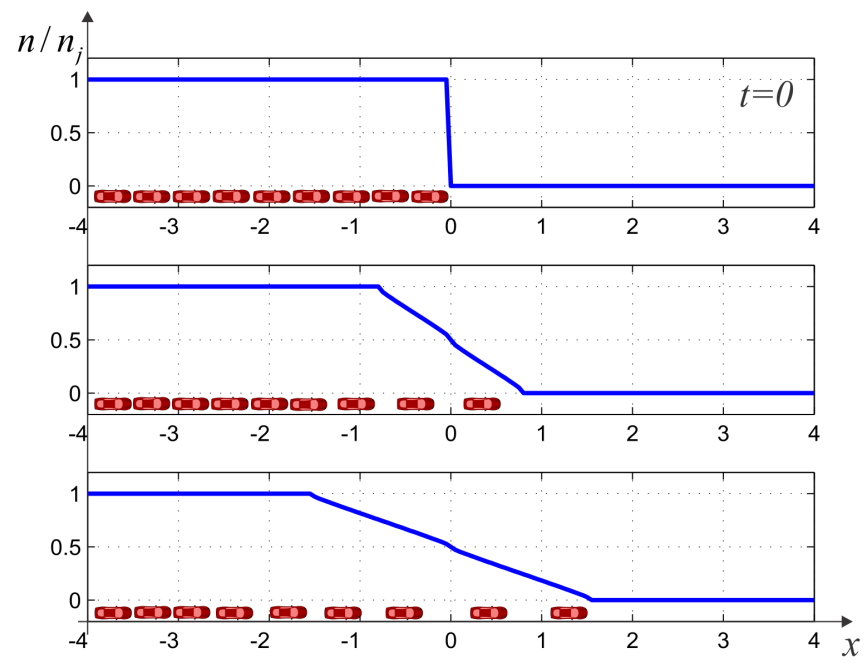

Figure 5.4.2: Typical profile of traffic density at subsequent times when traffic light turns green.

A discrete formulation of (5.5.1) on a computational domain $[0, X]$ is formulated below. The computational domain is divided into $N$ cells of length $\Delta x$ in a staggered way, with partition points

$x_{1 / 2}=0, x_{3 / 2}=\Delta x, \cdots, x_{i+1 / 2}=i \Delta x, \cdots, x_{N+1 / 2}=N \Delta x=X$. On the cell $C_{i}=\left[x_{i-1 / 2}, x_{i+1 / 2}\right]$, finite volume discretization of (5.3.2) reads

$$
n_{i}^{j+1}=n_{i}^{j}-\frac{\Delta t}{\Delta x}\left(F_{i+1 / 2}^{j}-F_{i-1 / 2}^{j}\right) .
$$

Equation (5.5.3) states that on every time step $t_{j}$, the value of $n_{i}$ is updated using the net flux from left and right boundaries $F_{i-1 / 2}^{j}$ and $F_{i+1 / 2}^{j}$. Here we will discuss two ways to approximate the flux, they are upwind method and Godunov.

\section{Upwind method}

In the upwind method, an approximation for the the flux $F_{i+\frac{1}{2}}$ is based on the sign of signal speed at the staggered point $x_{j+\frac{1}{2}}$. The signal speed at this staggered point $x_{j+\frac{1}{2}}$ is approximated by $\frac{1}{2}\left(f_{i}^{\prime}+f_{i+1}^{\prime}\right)$. Hence, the 


\subsection{FINITE VOLUME METHOD FOR THE KINEMATIC LWR MODEL77}

upwind approximation of $F_{i+\frac{1}{2}}$ is as follows

$$
F_{i+\frac{1}{2}} \approx \begin{cases}f_{i}, & \text { if } \frac{1}{2}\left(f_{i}^{\prime}+f_{i+1}^{\prime}\right)>0 \\ f_{i+1}, & \text { if } \frac{1}{2}\left(f_{i}^{\prime}+f_{i+1}^{\prime}\right)<0 .\end{cases}
$$

The upwind approximation above means the following, if the signal speed is positive then $f_{i+\frac{1}{2}}^{j} \approx f_{i}^{j}=n_{i}^{j} V_{m}\left(1-n_{i}^{j} / N_{m}\right)$, whereas if negative $f_{i+\frac{1}{2}}^{j} \approx$ $f_{i+1}^{j}=n_{i+1}^{j} V_{m}\left(1-n_{i+1}^{j} / N_{m}\right)$, see Figure 5.5.1. The above scheme is stable under the CFL-like condition $V_{m} \Delta t / \Delta x \leq 1$.
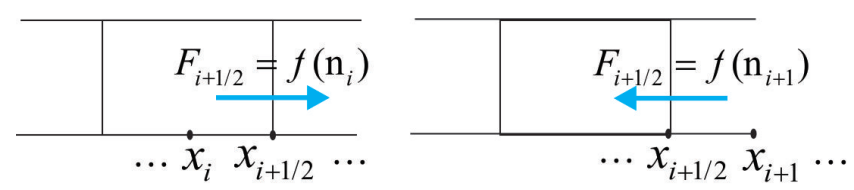

Figure 5.5.1: Upwind approximation for $F_{i+\frac{1}{2}}$, holds for every time iteration.

\section{Godunov method}

In Godunov method, the flux function $F_{i+\frac{1}{2}}$ is approximated using the analytical solution of the Riemann problem $u_{R}\left(0, n_{i}, n_{i+1}\right)$. To be explicit $F_{i+\frac{1}{2}}=u_{R}\left(1-u_{R}\right)$. Using (5.3.2), carefully formulate the following approximation

- If $n_{i}<n_{i+1}$, then

$$
F_{i+\frac{1}{2}}= \begin{cases}f\left(n_{i}\right), & \text { if } s_{i}>0 \\ f\left(n_{i+1}\right), & \text { if } s_{i}<0\end{cases}
$$

with

$$
s_{i}=\frac{f\left(n_{i+1}\right)-f\left(n_{i}\right)}{n_{i+1}-n_{i}}=\frac{1}{2}\left(f^{\prime}\left(n_{i}\right)+f^{\prime}\left(n_{i+1}\right)\right) .
$$

Note, this last equality only holds for Greenshield flux function.

- If $n_{i}>n_{i+1}$,

$$
F_{i+\frac{1}{2}}= \begin{cases}f\left(n_{i}\right), & \text { if } b\left(n_{i}\right)>0 \\ f(1 / 2), & \text { if } b\left(n_{i}\right)<0<b\left(n_{i+1}\right) \\ f\left(n_{i+1}\right), & \text { if } b\left(n_{i+1}\right)<0\end{cases}
$$




\section{Exercise 5.5.1.}

1. Implement the finite volume (5.5.3) with the flux function (5.5.4) to solve $(5.5 .1,5.5 .2)$ with initial condition

$$
u(x, 0)= \begin{cases}u_{\ell}, & \text { if } x<0, \\ u_{r}, & \text { if } x>0,\end{cases}
$$

(a) Simulate a shock wave solution emanated from initial solution (5.5.7) with $u_{\ell}=0.4, u_{r}=0.7$. Plot the result together with the analytical solution. Make sure that the shock wave propagates with the correct speed. Interpret this solution.

(b) Are there shock wave solutions that propagate to the right? Try to interpret this solution.

(c) Simulate a rarefaction wave solution emanated from (5.5.7) with $u_{\ell}=1, u_{r}=0$. Your simulation will typically like Figure 5.4.2. Plot your simulation result together with the analytical solution.

(d) Simulate a rarefaction wave solution emanated from (5.5.7), with $0<u_{\ell}<u_{r}<0.5$. Try to interpret this solution.

2. Repeat the above exercise using Godunov method. But first think carefully, how do you implement $(5.5 .5,5.5 .6)$ in your Matlab code. Which one is better upwind or Godunov? Provide a reasoning. 


\section{Chapter 6}

\section{The shallow water equations}

This chapter is about water waves, focusing on the mathematical model for free surface waves in shallow areas, i.e. the shallow water equation (SWE). The first two sections discuss two alternatives derivation of the SWE, followed with a section about derivation of the linear shallow water model and its dispersion relation. The last section discusses the finite difference method and the Riemann form of the linear SWE. A good understanding about the Riemann form of SWE turns out to be useful in formulating the numerical absorbing boundary conditions.

\subsection{Water waves and the shallow water model}

The general three-dimensional free surface flow for an incompressible fluid is governed by the Euler equations. These equations, however, cannot be solved efficiently with sufficient accuracy in complex geophysical flow domains. A set of simplified models are then derived from the Euler equations under various simplifying assumptions. In this preliminary course on fluid flow, we restrict the discussion on a lower dimensional problems.

Our discussion starts with the Euler equations, which is a particular form of the Navier Stokes equations holds for inviscid fluid with zero thermal conductivity. For a homogeneous fluid, the 2D Euler equation read as

$$
\begin{aligned}
u_{x}+w_{z} & =0, \\
u_{t}+u u_{x}+w u_{z} & =-g \eta_{x}-p_{x} \\
w_{t}+u w_{x}+w w_{z} & =-p_{z},
\end{aligned}
$$

with $(u, w)^{T}$ denotes the fluid particle velocity, and $p$ the hydrodynamic pressure term, see Figure 6.1.1. All these dependent variables are functions of $(x, z, t)$. Even in this simplified equations, formulating solutions is 
already a difficult task.

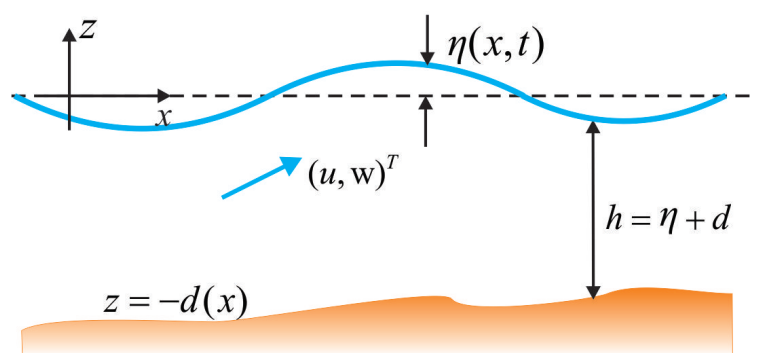

Figure 6.1.1: Sketch of fluid domain.

For systems in which the horizontal length scale is much greater than the vertical length, a depth integrated form of the Euler equations is much simpler. For horizontally dominant flow, continuity equation can be written as a dynamic equation in terms of surface elevation $\eta(x, t)$. This will be discussed below.

First, integrating (6.1.1) over the fluid depth from $z=-d(x)$ to $z=\eta(x, t)$ reads

$$
\begin{aligned}
& \left.\int_{-d(x)}^{\eta(x, t)} u_{x} d z+w\right]_{-d(x)}^{\eta(x, t)}=0, \\
& \int_{-d(x)}^{\eta(x, t)} u_{x} d z+w_{s}-w_{b}=0 .
\end{aligned}
$$

Consider fluid particles at the free surface $z=\eta(x, t)$. Total differentiation with respect to time $t$ reads

$$
\partial_{t} z=\partial_{t} \eta+\partial_{t} x \partial_{x} \eta
$$

Then, fluid particles velocity at the surface $\left(u_{s}, w_{s}\right)$ satisfies

$$
w_{s}=\eta_{t}+u_{s} \eta_{x}, \quad \text { along } z=\eta(x, t) .
$$

Relation (6.1.5) is known as the kinematic boundary condition. Similarly, along the impermeable bottom $z=-d(x)$, velocity vectors of fluid particles $\left(u_{b}, w_{b}\right)$ satisfy the following boundary condition

$$
w_{b}=-u_{b} d_{x}, \text { along } z=-d(x),
$$

which is called as the impermeable bottom boundary condition. 
In shallow water assumption, horizontal velocity of fluid particles is independent of $z$, and here we denote it as $\bar{u}(x, t)$. This assumption will directly lead to

$$
\int_{-d(x)}^{\eta(x, t)} u d z=\left.\bar{u} z\right|_{-d(x)} ^{\eta(x, t)}=\bar{u}(\eta+d)=\bar{u} h,
$$

with $h(x, t)=\eta(x, t)+d(x)$ represents the total water thickness, see Figure 6.1.1. Further, implementing the Leibniz rule will lead to

$$
\frac{d}{d x}\left(\int_{-d(x)}^{\eta(x, t)} u d z\right)=\int_{-d(x)}^{\eta(x, t)} u_{x} d z+u_{s} \eta_{x}-u_{b}\left(-d_{x}\right) .
$$

After using $(6.1 .5,6.1 .6)$ to replace $u_{s}$ and $u_{b}$, and using the definition of $\bar{u}$ we get

$$
\frac{d}{d x}(h \bar{u})=\int_{-d(x)}^{\eta(x, t)} u_{x} d z+w_{s}-\eta_{t}-w_{b} .
$$

Adopting the above relation, equation (6.1.4) can be further simplified to

$$
\eta_{t}+(h \bar{u})_{x}=0
$$

This equation is infact the mass conservation, that basically governs the dynamics of surface profile $\eta(x, t)$ as time elapsed. Since bottom topography $d(x)$ is independent to $t$, the above equation can also be written as

$$
h_{t}+(h \bar{u})_{x}=0 \text {. }
$$

Hence, equation (6.1.7) is the mass conservation for horizontal dominan flow. Further, we stated here without prove that the depth integrated momentum equation reads

$$
(h \tilde{u})_{t}+\left(h \tilde{u}^{2}\right)_{x}+g h \eta_{x}=p_{x} h .
$$

If we neglect hydrodynamic pressure $p$, the equations reduce to

$$
\begin{aligned}
h_{t}+(h \tilde{u})_{x} & =0, \\
(h \tilde{u})_{t}+(h \tilde{u} \tilde{u})_{x}+g h \eta_{x} & =0 .
\end{aligned}
$$

Equations $(6.1 .8,6.1 .9)$ are written in the form of conservative equations. Numerical method for solving $(6.1 .8,6.1 .9)$ while preserving their conservative property are considered as conservative schemes. This equation is known as the shallow water equations abbreviated as SWE. It consists of mass conservation (6.1.8) and momentum balance (6.1.9), with dependent variables: water thickness $h(x, t)$ and horizontal velocity $\tilde{u}(x, t)$.

Task: Show that $(6.1 .8,6.1 .9)$ are equivalent to

$$
\begin{aligned}
h_{t}+(h \tilde{u})_{x} & =0, \\
\tilde{u}_{t}+\tilde{u} \tilde{u}_{x}+g \eta_{x} & =0 .
\end{aligned}
$$




\subsection{Direct derivation of the shallow water equations}

In this section we present a direct derivation of the shallow water equations, starting from the two basic principles: mass conservation and momentum balance equations. Figure 6.2.1 displays a fluid layer, bounded above by free surface, and below by bottom topography. Fluid particle trajectories are shown in three different cases: deep water wave, shallow water wave, and transitional wave. Generally, fluid particles velocity is determined by a vector

$$
\mathbf{v}(x, z, t)=(u(x, z, t), w(x, z, t))^{T} .
$$

Task: Argue that for the case of shallow water depth, the following is a relevant approximation

$$
\mathbf{v}(x, z, t)=(u(x, t), 0)^{T} .
$$

\section{Mass conservation}

Consider a layer of fluid above bottom topography $z=-d(x)$. At any time the fluid surface is given by $\eta(x, t)$. We assume that velocity of fluid particles in the fluid domain is

$$
\mathbf{v}(x, z, t)=(u(x, t), 0)^{T},
$$

with the horizontal component $u(x, t)$, and zero vertical component. This is the shallow water assumption, in which the vertical component is neglected, and horizontal component are assumed to be the same throughout the entire fluid depth. This assumption is reasonable in shallow water area.

Take a control volume as depicted in Figure 6.2.2, the left and right boundary are $x_{1}$ and $x_{2}$, each moves with the horizontal flow $d x_{1} / d t=u\left(x_{1}, t\right)$, and $d x_{2} / d t=u\left(x_{2}, t\right)$, respectively. The mass of fluid in the control volume is

$$
M(t)=\int_{x_{1}(t)}^{x_{2}(t)} \rho(\eta+d) d x,
$$

with $\rho$ is the fluid density. The derivative of $M$ with respect to $t$ is

$$
\frac{d M}{d t}=\int_{x_{1}(t)}^{x_{2}(t)}(\rho(\eta+d))_{t} d x+\left.\rho(\eta+d)\right|_{x_{2}} \frac{d x_{2}}{d t}-\left.\rho(\eta+d)\right|_{x_{1}} \frac{d x_{1}}{d t} .
$$




\subsection{DIRECT DERIVATION OF THE SHALLOW WATER EQUATIONS83}

Shallow water wave, depth $\leq \lambda / 20$

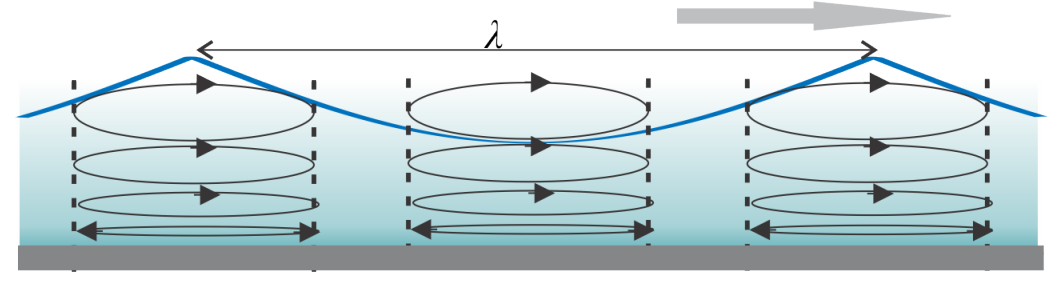

Transitional wave, $\lambda / 20<$ depth $\leq \lambda / 2$

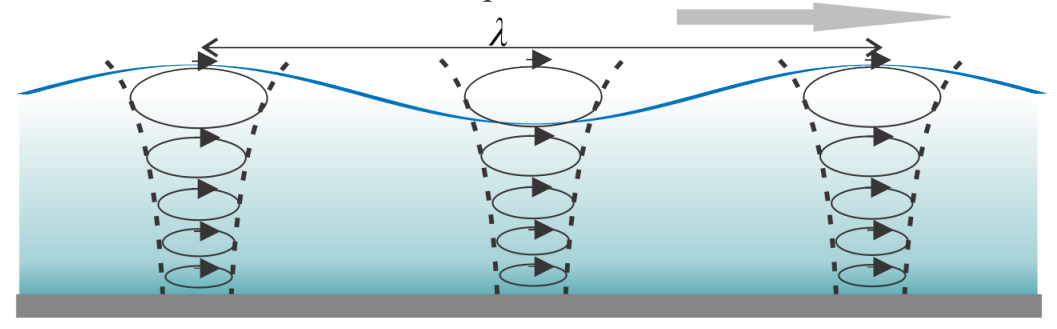

Deep water wave, depth $>\lambda / 2$

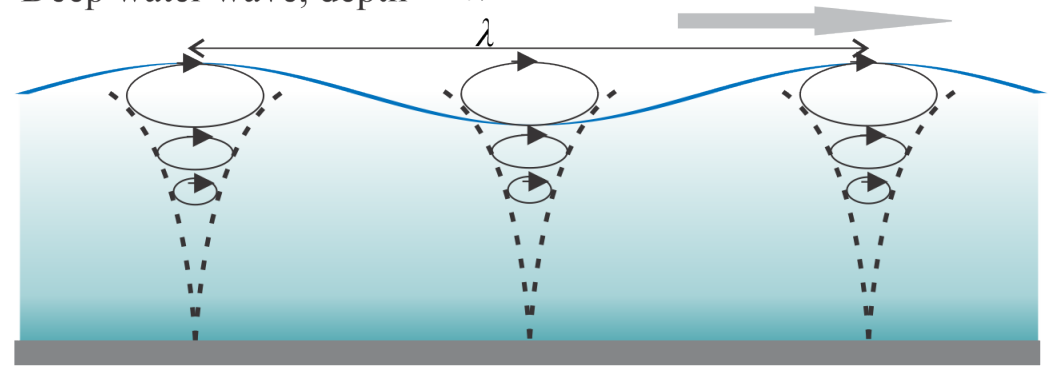

Figure 6.2.1: Wave classification based on depth and wavelength.

Since $d x_{i} / d t=u_{i}(t), i=1,2$, the last two terms on the right hand side can be written as

$$
\int_{x_{1}(t)}^{x_{2}(t)}(\rho(\eta+d) u)_{x} d x .
$$

If there is no fluid added to or subtracted from that control volume, then $\frac{d M}{d t}=0$ or

$$
\int_{x_{1}(t)}^{x_{2}(t)}(\rho h)_{t}+(\rho h u)_{x} d x=0 .
$$

Since the above equation holds for every choice of $x_{1}$ and $x_{2}$, then we get 


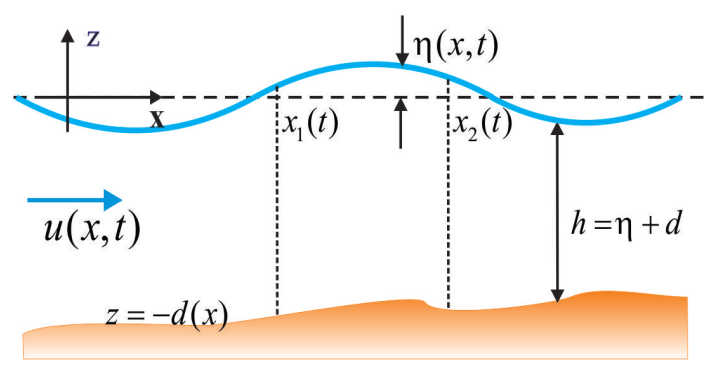

Figure 6.2.2: A control volume in the derivation of SWE.

the following mass balance equation

$$
(\rho h)_{t}+(\rho h u)_{x}=0 .
$$

\section{Momentum balance}

The Newton second law stated, the rate of change of momentum equals to the total force. Consider a fluid column as in Figure 6.2.2, total momentum of the fluid column is

$$
I(t)=\int_{x_{1}(t)}^{x_{2}(t)} \rho(\eta+d) u d x,
$$

and its rate of change is

$$
\frac{d I}{d t}=\int_{x_{1}(t)}^{x_{2}(t)}(\rho h u)_{t}+\left(\rho h u^{2}\right)_{x} d x .
$$

Forces are gravity force (or body force), pressure difference, and frictional force. We assume frictional force has magnitude proportional to velocity square, and has opposite direction to velocity:

$$
F_{S}=\int_{x_{1}(t)}^{x_{2}(t)}-\rho C_{f}|u| u d x .
$$

Gravitational force is proportional fluid mass, or explicitly

$$
F_{b o d y}=\int_{x_{1}(t)}^{x_{2}(t)} \rho g h d_{x} d x .
$$

Note that body force is nonzero only when $d(x) \neq$ constant, and it has the same direction with increasing $d(x)$, as expected. Next, we will formulate the pressure difference. Pressure at a position $(x, z)$ in the fluid domain is 
given by $p(x, z, t)=P_{a t m}+\rho g(\eta-z)$. Note that at the surface $z=\eta(x, t)$, the pressure is $P_{a t m}$ and it increases with increasing vertical position $-z$ till it reach its maximum at the bottom $z=-d(x)$. The total pressure on side $x_{i}$ is given by

$$
P\left(x_{i}, t\right)=\int_{-d\left(x_{i}\right)}^{\eta\left(x_{i}, t\right)} P_{a t m}+\rho g(\eta-z) d z=\frac{1}{2} \rho g h^{2},
$$

after taking $P_{a t m}$ to be zero. Pressure difference between left and right boundary is

$$
P\left(x_{1}, t\right)-P\left(x_{2}, t\right)=-\int_{x_{1}(t)}^{x_{2}(t)}\left(\frac{1}{2} \rho g h^{2}\right)_{x} d x .
$$

Hence, the momentum balance is

$$
\int_{x_{1}(t)}^{x_{2}(t)}(\rho h u)_{t}+\left(\rho h u^{2}\right)_{x} d x=\int_{x_{1}(t)}^{x_{2}(t)}\left(\rho g h d_{x}\right)-\int_{x_{1}(t)}^{x_{2}(t)}\left(\frac{1}{2} \rho g h^{2}\right)_{x}-\int_{x_{1}(t)}^{x_{2}(t)} \rho C_{f}|u| u
$$

or

$$
(\rho h u)_{t}+\left(\rho h u^{2}+\frac{1}{2} \rho g h^{2}\right)_{x}=(\rho g h) d_{x}-\rho C_{f}|u| u
$$

Hence, the shallow water equations for fluid with constant density $\rho$ is as follows

$$
\begin{aligned}
h_{t}+(h u)_{x} & =0 \\
(h u)_{t}+\left(h u^{2}+\frac{1}{2} g h^{2}\right)_{x} & =+d_{x}(g h)-C_{f}|u| u
\end{aligned}
$$

If we expand $(h u)_{t}=h_{t} u+h u_{t}$, and use continuity equation (6.2.7) to replace $h_{t}$ the equivalent form of shallow water equations are

$$
\begin{aligned}
\eta_{t}+(h u)_{x} & =0 \\
u_{t}+u u_{x}+g \eta_{x} & =-C_{f} \frac{u|u|}{h},
\end{aligned}
$$

after implementing $h_{t}=(\eta+d)_{t}=\eta_{t}$ for the case of no bottom motion.

\section{Exercise 6.2.1.}

1. Formulate the 1-dimensional SWE when there is an additional horizontal current $U(x, t)$. 


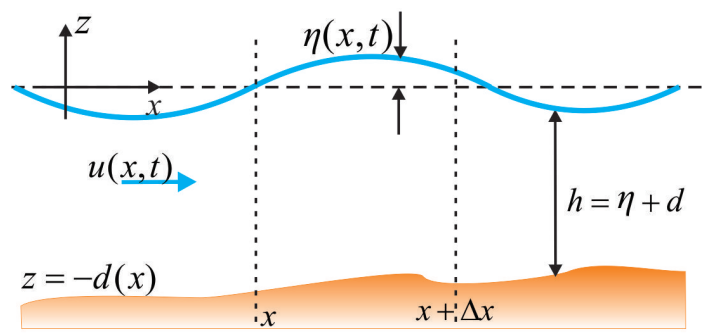

2. This exercise discusses an alternative way of continuity equation derivation. Consider a control volume bounded left and right by $x$, and $x+\Delta x$, respectively, see figure below. Shallow water assumption: horizontal velocity of fluid flow is $u(x, t)$. After a time interval $\triangle t$

$$
\begin{aligned}
\text { Input: } & \left.u(\eta+d)\right|_{x} \Delta t \\
\text { Output: } & \left.u(\eta+d)\right|_{x+\Delta x} \Delta t \\
\text { Storage: } & \left.(\eta+d)\right|_{t+\Delta t}-\left.(\eta+d)\right|_{t}
\end{aligned}
$$

Combining, input - output $=$ storage, will yield

$$
\left.u(\eta+d)\right|_{x} \Delta t-\left.u(\eta+d)\right|_{x+\Delta x} \Delta t=\left.(\eta+d)\right|_{t+\Delta t}-\left.(\eta+d)\right|_{t}
$$

We divide the equation above with $\Delta x \Delta t$ and taking the limit $\Delta t \rightarrow 0$, $\Delta x \rightarrow 0$ we get

$$
h_{t}+(h u)_{x}=0 .
$$

3. Consider the transport of a dissolved substance moving along a 1dimensional channel with cross section $A(x)$, with velocity $u(x, t)$, and $c(x, t)$ is the mean concentration in the cross-section. Formulate the mass balance equation for a dissolved substance using a control volume $\left[x_{1}(t), x_{2}(t)\right]$, in which boundaries of the control volume $x_{1}(t)$ and $x_{2}(t)$ move with the flow $\frac{d x_{i}}{d t}=u\left(x_{i}, t\right)$, for $i=1,2$, so that no substance get in or out because of transport phenomena. But since it is a dissolved substance, the substance may get in or out through the left and right moving boundaries, due to a socalled diffusive flux (When the concentration $c$ is not constant over $x$, there will be a mass flux trying to level out the differences.) According to Fick's law, this flux $\Phi$ is proportional to the gradient of $c$ 


$$
\Phi(x, t)=-D \frac{\partial c}{\partial x}
$$

with $D$ is the diffusion coefficient. Show that in this case the mass balance equation is

$$
\frac{\partial(A c)}{\partial t}+\frac{\partial(A c u)}{\partial x}-D \frac{\partial\left(A c_{x}\right)}{\partial x}=0
$$

while for a constant cross section $A$, it becomes

$$
\frac{\partial c}{\partial t}+\frac{\partial(c u)}{\partial x}-D \frac{\partial^{2} c}{\partial x^{2}}=0
$$

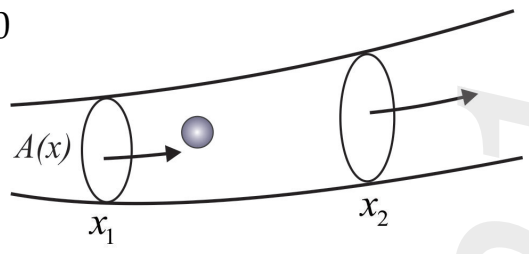

\subsection{Linear Shallow Water Equations}

The simplest form of the shallow water equations are when the bottom is flat and when the nonlinear terms are negligible. Here, we will discuss the physical situation in which it applies. Consider the 1-D shallow water equations for flat bottom $z=-d_{0}$, without friction

$$
\begin{aligned}
\eta_{t}+\left(\left(\eta+d_{0}\right) u\right)_{x} & =0 \\
u_{t}+u u_{x}+g \eta_{x} & =0 .
\end{aligned}
$$

Show that $\eta(x, t)=0$ and $u(x, t)=u_{0}$, with a constant $u_{0}$, are steady solutions for the SWE $(6.1 .8,6.1 .9)$. That means the undisturbed water level with ambient current $u_{0}$, is the steady situation.

The linear shallow water equations is a model for small deformation of the steady situation. Suppose $\hat{\eta}(x, t)$ and $\hat{u}(x, t)$ are perturbations of $\eta(x, t)$ and $\tilde{u}(x, t)$ with respect to the steady situation $\eta(x, t)=0$, and $u(x, t)=u_{0}$

$$
\eta(x, t)=0+\varepsilon \hat{\eta}(x, t), \quad \tilde{u}(x, t)=u_{0}+\varepsilon \hat{u}(x, t),
$$

with $\varepsilon$ is a small parameter. Substituting $(6.3 .3)$ into $(6.1 .8,6.1 .9)$ will result equations with terms of consecutive order $\mathcal{O}(\varepsilon)$ and $\mathcal{O}\left(\varepsilon^{2}\right)$. Neglecting high order terms $\mathcal{O}\left(\varepsilon^{2}\right)$ will give us the following equations

$$
\hat{\eta}_{t}+u_{0} \hat{\eta}_{x}+d_{0} \hat{u}_{x}=0, \quad \hat{u}_{t}+u_{0} \hat{u}_{x}+g \hat{\eta}_{x}=0,
$$

which is a linear SWE under ambient current $u_{0}$. Equations above can be further simplified by adopting a moving coordinate $\hat{x}=x-u_{0} t$, and $\hat{t}=t$.

$$
\hat{x}=x-u_{0} t, \quad \hat{t}=t .
$$


Re-writting (6.3.4) in terms of new independent variables will result in the simplest linear SWE as follows

$$
\begin{aligned}
& u_{t}+g \eta_{x}=0, \\
& \eta_{t}+d_{0} u_{x}=0,
\end{aligned}
$$

in which all hats have been erased. Also for ease of notations, in further discussions, all variables $\eta, u$ also $x, t$ will be written without hats. However, we always have to be aware the linear SWE holds for waves with relatively small amplitude and velocity.

Further, one can show that $(6.3 .6,6.3 .7)$ can be decoupled into two standard wave equations

$$
\begin{aligned}
& \eta_{t t}-g d_{0} \eta_{x x}=0, \\
& u_{t t}-g d_{0} u_{x x}=0 .
\end{aligned}
$$

Since the linear SWE $(6.3 .6,6.3 .7)$ is equivalent with a pair of wave equations $(6.3 .8,6.3 .9)$, they also have the same left and right running characteristics with gradient $\frac{d x}{d t}= \pm c_{0}$, as depicted in Figure 6.3.1.

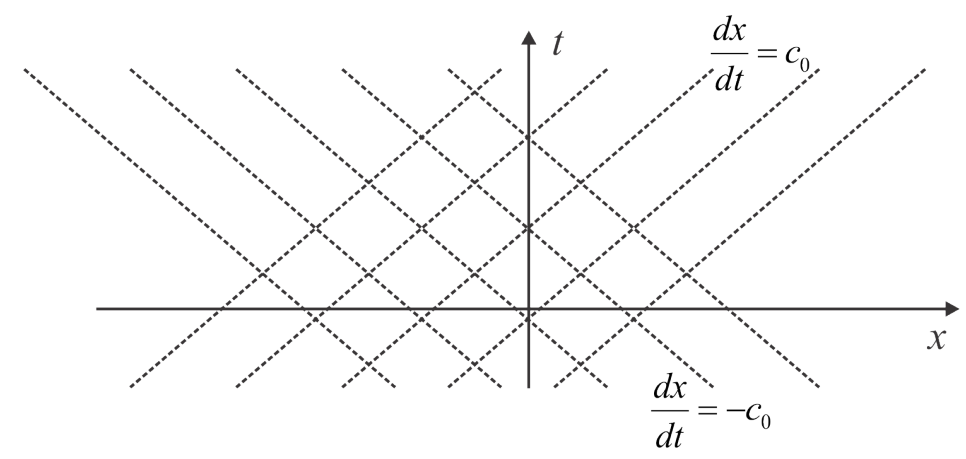

Figure 6.3.1: Left and right running characteristic of the linear SWE (6.3.6, 6.3.7)

\section{Dispersion relation for the linear SWE}

Next, we will discuss the property of SWE solution. First, note that the SWE $(6.3 .6,6.3 .7)$ are equivalent with the wave equations $(6.3 .8,6.3 .9)$. Substituting a monochromatic wave like form $\eta(x, t)=\exp i(k x-\omega t)$ into (6.3.8) will yield

$$
\left(-\omega^{2}+k^{2} g d_{0}\right) \exp i(k x-\omega t)=0 .
$$


The above equation will be satisfied if $\omega$ and $k$ obey

$$
\left(\frac{\omega}{k}\right)^{2}=g d_{0}
$$

Relation (6.3.11) is called the dispersion relation, which should be satisfied by all solutions of (6.3.8). Please note that phase velocity of the monochromatic wave (6.3.10) is $\frac{\omega}{k}$, hence solution of the governing equation (6.3.8) possess wave property: the signal will propagate to the left and right with phase velocity $c_{0}= \pm \sqrt{g d_{0}}$, according to d'Alembert solution. This phase velocity holds for all monochromatic waves of any wave number $k$. This phase velocity depends solely on the water depth $d_{0}$, i.e. a wave on a deeper depth has larger velocity. The same property also holds for $u(x, t)$.

In this course, we restrict our discussion on the numerical scheme for shallow water equations. Starting with the simplest scheme for the SWE linear, then we attack the half linear SWE, and finally the full non linear SWE. All this schemes are considered as hydrostatic schemes, this is because in these SWE formulation, we take into account hydrostatic pressure only, whereas hydrodynamic pressure is neglected. It is to be noted that by adopting shallow water model, each wave component has the same phase velocity $\pm \sqrt{g d_{0}}$ as a consequence of the dispersion relation (6.3.11). For water wave problems over arbitrary depth (including shallow and deep water), the exact dispersion relation should be

$$
\omega^{2}=g k \tanh k d_{0}
$$

This exact dispersion relation can be derived from the full Euler equations, see [4] Subsection 3.4. In order to have a numerical scheme that can properly describes phase velocity of waves in deeper water area, an approximation of the Euler equations shall be considered, and numerical schemes are called non-hydrostatic schemes. This non-hydrostatic modelling is still an active area of research.

\section{Exercise 6.3.1.}

1. (Standing wave) Consider $(6.3 .6,6.3 .6)$ on a domain $x \in \mathbb{R}, t>0$, with an initial condition $\eta(x, 0)=\cos (\pi x / L), u(x, 0)=0$. Show that $\eta(x, t)=\cos (\pi x / L) \cos \left(\pi c_{0} t / L\right)$ is the analytical solution. Next, find the corresponding solution $u(x, t)$.

2. Find dispersion relation of the linear SWE directly from $(6.3 .6,6.3 .7)$ by assuming wave like solutions in the form of $\eta(x, t)=h_{0} \exp i(k x-$ 
$\omega t), u(x, t)=u_{0} \exp i(k x-\omega t)$. Hint: Dispersion relation can be obtained as a necessary condition for $(6.3 .6,6.3 .7)$ to have a non-trivial solutions.

3. Substituting the wave like solution $u(x, t)=u_{0} \exp i(k x-\omega t)$ into

$$
u_{t}=-d u_{x}+k u_{x x}, \quad \text { with } \quad k>0,
$$

to find a dispersion relation. Discuss the damping behaviour of the solution.

4. Find dispersion relation of the following equations

$$
u_{t}=-d u_{x}+a u_{x x x} .
$$

Show that it directly determines the phase velocity of the wave $\frac{\omega}{k}=$ $d-k^{2}$. In this case, each monochromatic wave with wave number $k$ moves with different phase speed.

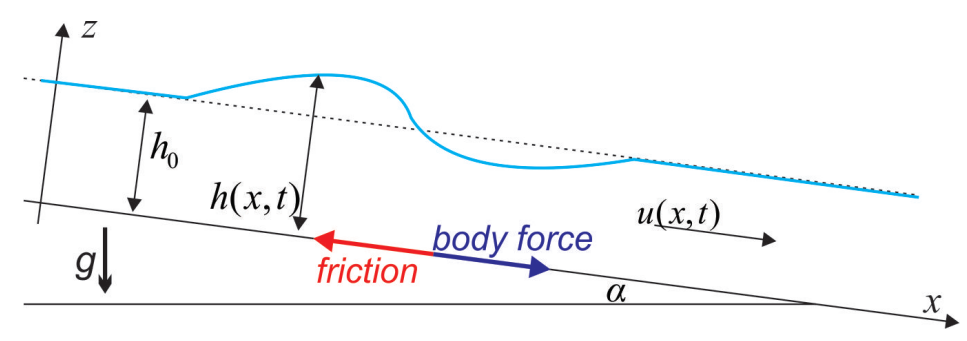

Figure 6.3.2: Sketch of an inclined channel and notations.

5. (Inclined channel flow) Consider an open channel with constant slope $\alpha$ with a rectangular cross section. The water height $h(x, t)$ and horizontal velocity $u(x, t)$ satisfy the so-called Saint-Venant Equations:

$$
\begin{aligned}
\partial_{t} h+\partial_{x}(u h) & =0 \\
\partial_{t}(h u)+\partial_{x}\left(h u^{2}+\frac{1}{2} g h^{2}\right) & =g h \sin \alpha-C_{f}|u| u, \\
\text { Manmirg } &
\end{aligned}
$$

with $C_{f}$ is the Darey Weisbach friction coefficient. For relatively small bottom inclination, we often approximate $\sin \alpha \approx \alpha$.

(a) In steady situations the gravitational force balances the friction force, find the relation of this steady fixed water height $h_{0}$ and velocity $u_{0}$. Answer: $g h_{0} \alpha=C_{f}\left|u_{0}\right| u_{0}$ 
(b) Introduce a small deviation of the steady situation

$$
\left(\begin{array}{l}
h \\
u
\end{array}\right)=\left(\begin{array}{l}
h_{0}+\varepsilon \hat{h}(x, t) \\
u_{0}+\varepsilon \hat{u}(x, t)
\end{array}\right)
$$

Formulate the linearized model of $(6.3 .13,6.3 .14)$ that holds for small deviation around the steady solution.

6. (Blood flow) Blood moves because of the pressure developed in the heart during pumping. If we assume the blood flow is not too fast and the artery is not deformed too much, blood flow is governed by

$$
\frac{\partial^{2} v}{\partial t^{2}}-c^{2} \frac{\partial^{2} v}{\partial x^{2}}=-\frac{1}{\rho} \frac{\partial^{2} P}{\partial x \partial t}
$$

here $v(x, t)$ denotes flow velocity, and $P(x, t)$ pressure at arteri due to muscle motion. Suppose $p(x, t)=P_{0}(t-x / w)$, show that the solution of (6.3.16) is

$$
v(x, t)=\frac{\omega}{\rho\left(\omega^{2}-c^{2}\right)} P_{0}(t-x / w)+f(x-c t)+g(x+c t),
$$

with the first term being the respon of the muscle motion.

\subsection{Finite difference method}

The simplest finite difference method or the linear SWE is the Lax method, as follows

$$
\begin{aligned}
u_{j}^{n+1} & =\frac{1}{2}\left(u_{j+1}^{n}+u_{j-1}^{n}\right)-g \frac{\Delta t}{2 \Delta x}\left(\eta_{j+1}^{n}-\eta_{j-1}^{n}\right) \\
\eta_{j}^{n+1} & =\frac{1}{2}\left(\eta_{j+1}^{n}+\eta_{j-1}^{n}\right)-d_{0} \frac{\Delta t}{2 \Delta x}\left(u_{j+1}^{n}-u_{j-1}^{n}\right)
\end{aligned}
$$

This equation is of order $\mathcal{O}\left(\Delta t, \Delta x^{2}\right)$ (prove this). For a second order scheme, of order $\mathcal{O}\left(\Delta t^{2}, \Delta x^{2}\right)$, commonly adopted scheme are: Lax-Wendroff, leapfrog, or Mac-Cormack, for reference see [6], Section 15.

Stability condition of $(6.4 .1,6.4 .2)$ will be discussed below. A von Neumann stability analysis must consider both equations simultaneously, because they are coupled. Substituting both Fourier components

$$
\eta_{j}^{n}=\xi^{n} e^{i \theta}, \quad u_{j}^{n}=\mu^{n} e^{i \theta}, \text { with } \theta=k \Delta x
$$


into (6.4.1, 6.4.2), and after simplifying the equations one will get

$$
\left(\begin{array}{l}
\xi^{n+1} \\
\mu^{n+1}
\end{array}\right)=\left(\begin{array}{cc}
\cos \theta & -i c_{1} \sin \theta \\
-i c_{2} \sin \theta & \cos \theta
\end{array}\right)\left(\begin{array}{l}
\xi^{n} \\
\mu^{n}
\end{array}\right)
$$

with $c_{1}=g \Delta t / \Delta x, c_{2}=d_{0} \Delta t / \Delta x$. For the difference equations (6.4.1, 6.4.2), to be stable, the amplification matrix

$$
A=\left(\begin{array}{cc}
\cos \theta & -i c_{1} \sin \theta \\
-i c_{2} \sin \theta & \cos \theta
\end{array}\right)
$$

must have a spectral radius which is less than one. Solving the following equation for the eigenvalues of $A$ :

$$
\left|\begin{array}{cc}
\cos \theta-\lambda & -i c_{1} \sin \theta \\
-i c_{2} \sin \theta & \cos \theta-\lambda
\end{array}\right|=0
$$

to yield formula for eigenvalues

$$
\lambda=\cos \theta \pm i C \sin \theta, \text { with } C=\sqrt{c_{1} c_{2}}=\sqrt{g d_{0}} \frac{\Delta t}{\Delta x} .
$$

The magnitude of $\lambda$ is

$$
|\lambda|=\left(\cos ^{2} \theta+C^{2} \sin ^{2} \theta\right)^{1 / 2}=\left(1-\sin ^{2} \theta\left(1-C^{2}\right)\right)^{1 / 2} .
$$

Since $\sin ^{2} \theta \geq 0$ for all values of $\theta=k \Delta x,|\lambda|$ will be less than unity if the Courant number $C \leq 1$, or explicitly

$$
C=\sqrt{g d_{0}} \frac{\Delta t}{\Delta x} \leq 1
$$

Condition (6.4.3) is the stability condition for the Lax scheme for the linear SWE.

$\%$ Pseudo code: Lax scheme for the linear SWE on $0<x<L, t>0$

$\%$ with hard-wall left and right boundaries $u(0, t)=0, u(L, t)=0$,

$\%$ the corresponding boundary conditions for $\eta$ are $\eta_{x}(0, t)=0, \eta_{x}(L, t)=$ 0 ,

$\%$ see the discussion about Boundary Conditions on page 96.

$\% \%$ Initialization

$\%$ Determine $\mathrm{e}(:, 1)$ and $\mathrm{u}(:, 1)$ from the initial conditions

$\%$ In the following we compute $\mathrm{e}(:, \mathrm{n}+1)$ and $\mathrm{u}(:, \mathrm{n}+1)$ for every time step for $\mathrm{n}=1: \mathrm{Nt}-1$

$$
\mathrm{u}(1, \mathrm{n}+1)=0 ; \% \text { hard wall left boundary } u(0, t)=0
$$


for $\mathrm{j}=2: \mathrm{Nx} \%$ One loop for computing $u 1(2: N x)$ $\mathrm{u}(\mathrm{j}, \mathrm{n}+1)=\frac{1}{2}(\mathrm{u}(\mathrm{j}+1, \mathrm{n})+\mathrm{u}(\mathrm{j}-1, \mathrm{n}))-\mathrm{g}^{*} \mathrm{dt} /(2 \mathrm{dx})^{*}(\mathrm{e}(\mathrm{j}+1, \mathrm{n})-\mathrm{e}(\mathrm{j}-1, \mathrm{n})) ;$

end

$\mathrm{u}(\mathrm{Nx}+1, \mathrm{n}+1)=0 ; \%$ hard wall right boundary $u(L, t)=0$

$\mathrm{e}(1, \mathrm{n}+1)=\mathrm{e}(2, \mathrm{n})-\mathrm{d}^{*} \mathrm{dt} / \mathrm{dx} \mathrm{x}^{*} \mathrm{u}(2, \mathrm{n}) ; \%$ hard wall left boundary $\eta_{x}(0, t)=0$ for $\mathrm{j}=2: \mathrm{Nx}-1 \%$ One loop for computing $e 1(1: N x)$

$e(j, n+1)=\frac{1}{2}(e(j-1, n)+e(j+1, n))-d^{*} d t /(2 d x)^{*}(u(j+1, n)-u(j-1, n)) ;$

end

0

$\mathrm{e}(\mathrm{Nx}, \mathrm{n}+1)=\mathrm{e}(\mathrm{Nx}, \mathrm{n})-\mathrm{d}^{*} \mathrm{dt} / \mathrm{dx} \mathrm{x}^{*} \mathrm{u}(\mathrm{Nx}, \mathrm{n}) ; \%$ hard wall right boundary $\eta_{x}(L, t)=$

$\%$ Plot the result

figure(1)

end

plot(xplot,e(:,n+1)); \% or plot(xplot, $\mathrm{u}(:, \mathrm{n}+1))$

\section{Exercise 6.4.1.}

1. (Standing wave simulation) Implement the Lax method to solve (6.3.6, 6.3.6) in a domain $0<x<L, t>0$. Take a hard-wall type for the left and right boundary $u(0, t)=0, u(L, t)=0$. Take an initial condition $\eta(x, 0)=\cos (\pi x / L)$, and simulate the back and forth motion of surface in a form of standing wave in a closed basin. Find the period and frequency of the calculated motion. Make sure that the calculated frequency confirm the analytical frequency $\omega=k c_{0}=\pi c_{0} / L$, with $c_{0}=\sqrt{g d_{0}}$ is the phase velocity.

2. For the previous simulation, compute these two quantities: mass $M(t)=\int \eta d x$ and energy $E(t)=\int_{0}^{L} \frac{1}{2}\left(\eta_{t}^{2}+g d_{0} \eta_{x}^{2}\right) d x$. Are both quantities constant during the evolution?

3. Use the Lax scheme above to simulate the d'Alembert solution: starting from an initial hump, it will split into two exactly the same waves, one wave is running to the right, and the other is running to the left with constant velocities $c_{0}=\sqrt{g d_{0}}$.

4. Implement the Lax method for solving $(6.3 .6,6.3 .7)$ in a computational domain $0 \leq x \leq L, t>0$, subject to periodic boundary conditions $u(0, t)=u(L, t)$ and $\eta(0, t)=\eta(L, t)$. Simulate a monokromatik wave propagating to the right starting from an initial conditions $\eta(x, 0)=A \cos (8 \pi x / L), u(x, 0)=\sqrt{g / / d_{0}} \eta(x, 0)$.

5. Re-do all the exercises above using the second order Lax-Wendroff method. What improvements are apparent? 


\section{Exercise 6.4.2.}

(Pumping waves) In this exercise you should compose a code based on the linear SWE on $0<x<L$, and generates a monochromatic wave by pumping the middle part at $x=L / 2$. One way to achieve this purpose is by adopting an external force $f(x, t)=\delta(x-L / 2) 2 c_{0} s(t)$, with $s(t)$ denotes the wave signal to be generated. For generating a monochromatic wave with the respective amplitude and frequency $A$ and $\omega$, we can simply take

$$
\begin{aligned}
& \eta_{t}=-d_{0} u_{x}+\delta(x-L / 2) 2 c_{0} A \sin (\omega t) \\
& u_{t}=-g \eta_{x}
\end{aligned}
$$

On a computational domain $0<x<L, t>0$, solve the equations above with still water level as the initial conditions. Implement absorbing boundary conditions for both left and right boundary for $x=0$ and $x=L$. As result of the 'pumping' mechanism above, you will observe the appearance of monochromatic waves that propagates to the right and to the left with constant velocity $c_{0}$. What is the wavelength of the resulting wave? Check that this mechanism produce a monochromatic wave with wavelength $2 \pi / k$ that relate with the wave frequency $\omega$ according to dispersion relation of shallow water equations (6.3.11).

\section{Boundary conditions}

Here we will further discuss about a suitable boundary condition for the shallow water equation in order to formulate a well-posed problem. In Exercise 6.4.1 no. 1, the hard-wall left boundary is expressed as $u(0, t)=0$. Under this condition, how fluid particles along $x=0$ will move?

Consider $(6.3 .6,6.3 .6)$ on a domain $x \in \mathbb{R}, t>0$, subject to some initial conditions $\eta(x, 0)$ and $u(x, 0)$. Frequently used boundary conditions are the following

$$
\begin{array}{cl}
\text { hard-wall boundary } & u(0, t)=0, \quad u(L, t)=0, \\
\text { fixed ends } & \eta(0, t)=0, \quad \eta(L, t)=0, \\
\text { absorbing boundaries } & \text { to be discussed in Section 6.5, }
\end{array}
$$

or any other combinations of the above. For a certain physical condition that we consider, suitable boundary conditions can be chosen.

Task: Show that the following boundary conditions for the linear SWE (6.3.6) are equivalent

$$
u(0, t)=0 \quad \Leftrightarrow \quad \partial_{x} \eta(0, t)=0 .
$$


(Hint: differentiate the condition $u(0, t)=0$ with respect to time $t$, followed with substituting the result to (6.3.6) for $x=0$.)

Task: Can you use the following set of boundary conditions $u(0, t)=0$ and $\eta(0, t)=0$ to solve the linear SWE (6.3.6)? Explain your answer.

Someone tends to simply take zero boundary conditions for their first run, which is of course sensible. However, taking both $u(0, t)=0$ and $\eta(0, t)=0$ will lead to an ill-posed problem. This is because, once we take $u(0, t)=0$ for the linear SWE (6.3.6), it directly leads to $\partial_{x} \eta(0, t)=0$. So if at the same time we take $\eta(0, t)=0$, it will be conflicting, and the problem will become ill-posed. An easy way to remember this issue is by looking at waves in a pool with hard-wall boundary, when horizontal velocity is zero due to a hard wall $u(0, t)=0$, the free surface should be able to move instead of fixing it to zero by taking $\eta(0, t)=0$. Using the linear shallow water equations, we can further show that the surface has zero inclination $\eta_{x}(0, t)=0$. Finally, a suggestion for formulating correct boundary conditions is by taking into account the corresponding physical situation.

\subsection{Riemann invariant form}

In this section, we formulate the Riemann formulation of the shallow water equations. This formulation turns out to be very useful in understanding the main characteristics of SWE. Consider $(6.3 .6,6.3 .7)$ which is written in a matrix form

$$
\left(\begin{array}{l}
u_{t} \\
\eta_{t}
\end{array}\right)=\left(\begin{array}{cc}
0 & -g \\
-d_{0} & 0
\end{array}\right)\left(\begin{array}{l}
u_{x} \\
\eta_{x}
\end{array}\right)
$$

As a hyperbolic problem, the matrix coefficient of the linear SWE can be diagonalized

$$
\left(\begin{array}{cc}
0 & -g \\
-d_{0} & 0
\end{array}\right)=\Lambda\left(\begin{array}{cc}
-c_{0} & 0 \\
0 & c_{0}
\end{array}\right) \Lambda^{-1}, \quad c_{0}=\sqrt{g d_{0}} .
$$

with

$$
\lambda=\frac{1}{2}\left(\begin{array}{cc}
1 & 1 \\
\sqrt{d_{0} / g} & -\sqrt{d_{0} / g}
\end{array}\right), \quad \Lambda^{-1}=\left(\begin{array}{cc}
1 & \sqrt{g / d_{0}} \\
1 & -\sqrt{g / d_{0}}
\end{array}\right) .
$$

Then (6.5.1) becomes

$$
\Lambda^{-1}\left(\begin{array}{l}
u_{t} \\
\eta_{t}
\end{array}\right)=\Lambda^{-1}\left(\Lambda\left(\begin{array}{cc}
-c_{0} & 0 \\
0 & c_{0}
\end{array}\right) \Lambda^{-1}\right)\left(\begin{array}{l}
u_{x} \\
\eta_{x}
\end{array}\right) .
$$


If we denote $\left(\begin{array}{c}R_{+} \\ R_{-}\end{array}\right) \equiv \Lambda^{-1}\left(\begin{array}{c}u \\ \eta\end{array}\right)$ then relation between $R_{+}, R_{-}$in term of $u, \eta$ are

$$
\left(\begin{array}{l}
R_{+} \\
R_{-}
\end{array}\right)=\left(\begin{array}{cc}
1 & \sqrt{g / d_{0}} \\
1 & -\sqrt{g / d_{0}}
\end{array}\right)\left(\begin{array}{l}
u \\
\eta
\end{array}\right)=\left(\begin{array}{c}
u+\sqrt{g / d_{0}} \eta \\
u-\sqrt{g / d_{0}} \eta
\end{array}\right),
$$

and the linear SWE becomes

$$
\begin{aligned}
& \left(\partial_{t}+c_{0} \partial_{x}\right) R_{+}=0 \\
& \left(\partial_{t}-c_{0} \partial_{x}\right) R_{-}=0 .
\end{aligned}
$$

Moreover, the relation between $u, \eta$ in term of $R_{+}, R_{-}$are

$$
\left(\begin{array}{c}
u \\
\eta
\end{array}\right)=\Lambda\left(\begin{array}{l}
R_{+} \\
R_{-}
\end{array}\right)=\frac{1}{2}\left(\begin{array}{c}
R_{+}+R_{-} \\
\sqrt{d_{0} / g}\left(R_{+}-R_{-}\right)
\end{array}\right) .
$$
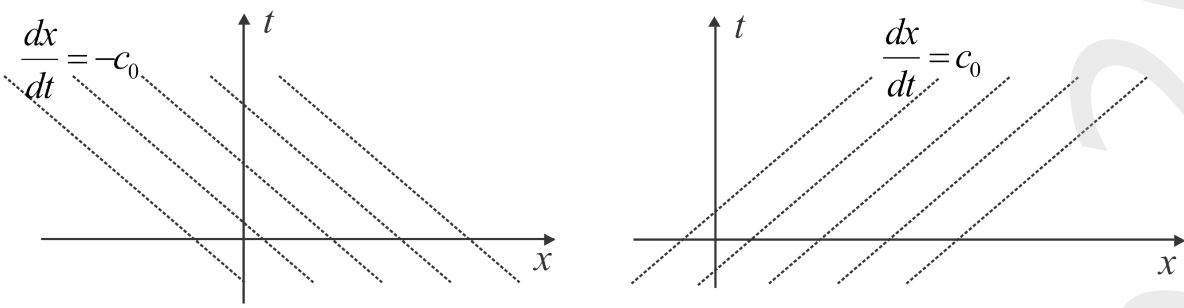

Figure 6.5.1: Left running characteristics with gradient $\frac{d x}{d t}=-c_{0}$ of (6.5.4) and right running characteristics with gradient $\frac{d x}{d t}=c_{0}$ of (6.5.3).

Equations $(6.5 .3,6.5 .4)$ are known as the Riemann invariant form of the SWE equation. These equations are the equivalent form of the SWE equations. In SWE $(6.3 .6,6.3 .7)$ equations for $\eta, u$ are coupled, but in Riemann form $(6.5 .3,6.5 .4)$, variables $R_{+}, R_{-}$are decoupled. In $(6.3 .6,6.3 .7)$, the variables $\eta, u$ carry information of both, the left and right running waves. Look closely to (6.5.3) and (6.5.4) which are transport equations, for the right and left running waves, respectively. Hence, we can deduce that the right information is carried solely by $R_{+}$, and the left information is carried solely by $R_{-}$. The corresponding characteristics are depicted in Figure 6.5.1.

Suppose we want to simulate the right running monochromatic wave with amplitude $A$ enters from the left boundary $x=0$ and propagates to the right, and further, passing through the right boundary $x=L$. Instead of 
using the SWE $(6.3 .6,6.3 .7)$, we can use their Riemann formulation. In Riemann formulation, since there is only right running wave, we can just take $R_{-}(x, t)=0$, which is equivalent with the relation $u(x, t)=\sqrt{g / d_{0}} \eta(x, t)$ (make sure on this!). Hence, for this simulation, we only need to calculate $R_{+}(x, t)$ from (6.5.3) using the FTBS method. This scheme needs the left boundary, and we should take $R_{+}(0, t)=2 \sqrt{g / d_{0}} A \sin \omega t$, in order to produce a monochromatic wave with amplitude $A$ (explain!). Do you need a right boundary condition to achieve the right absorbing boundary?

Exercise 6.5.1. In this exercise you should adopt the suitable finite difference method to solve the Riemann form of SWE $(6.5 .3,6.5 .4)$.

1. (Progressive wave) Simulate a monochromatic wave $A \sin \omega t$ influx enters from the left boundary, propagates in the domain with constant velocity $c_{0}=\sqrt{g d_{0}}$, and then leaves the right boundary. In this exercise, we deal with left wave influx boundary and right absorbing/transparent boundary.

2. (Reflected wave against a hard wall) Similar like (a) but on the right boundary, apply a hard wall right boundary $u(L, t)=0$. What is the phase difference between incoming and reflecting waves in this case? From your simulation, watch closely the reflected wave that propagates to the left till it arrived at $x=0$. Notice that you have a transparent boundary at $x=0$, because the right incoming wave can enter the domain, whereas the left reflected wave can leave the domain at the same time.

3. (Reflected wave against a fixed end) A monochromatic wave influx from left boundary propagates in a domain with fixed water level $\eta(L, t)=$ 0 . What is the phase difference between incoming and reflecting waves in this case?

4. (d'Alembert solution) Simulate the d'Alembert solution in which the initial wave splits into two exactly the same waves. One wave is running to the right, and the other is running to the left with constant velocities $c_{0}=\sqrt{g d_{0}}$. Implement the left and right absorbing boundaries.

Aside: For the Riemann formulation $(6.5 .3,6.5 .4)$, one left boundary condition for $R_{+}$is needed, also one right boundary for $R_{-}$. Of course, this property also holds for its equivalent form, the linear $\operatorname{SWE}(6.3 .6,6.3 .7)$, and that is; one boundary condition for each end $x=0$ and $x=L$ is needed. This conclusion is important in formulating a well-posed problem. 


\section{Exercise 6.5.2.}

Repeat the above exercises but now implement the Lax scheme of SWE $(6.4 .1,6.4 .2)$. Extra attention is needed for handling the suitable boundary conditions.

1. (Progressive wave) A suitable boundary condition for having an incoming wave from the left boundary is a set of

$$
\eta(0, t)=A \sin (\omega t), \quad u(0, t)=\sqrt{g / d_{0}} \eta(0, t) .
$$

Explain how do we arrive at this relation. Implement this formula on your Lax code of SWE and produce a wave entering from the left boundary, propagates into the domain and on your right you should implement an absorbing boundary. Test your formulation using your simulation.

2. (Reflected wave against a hard wall) For this simulation you can modify your program from number one to adopt the right hardwall boundary. Take a closer look of your simulation result, especially after the wave has reflected from the hardwall, and the reflected wave hit the left boundary (6.5.6). Once the reflected wave has arrived $x=0$, your simulation is not valid anymore. That is because at $x=0$ you cannot do both: adopting (6.5.6) and implementing absorbing boundary at the same time.

You can try to compose your transparent boundary for your Lax scheme of SWE, but it will require extra effort. 


\section{Chapter 7}

\section{Staggered grid method for SWE}

In this chapter we will discuss a numerical scheme for the shallow water equations which uses a staggered grid. The scheme is based on the finite difference leapfrog method. This staggered scheme turns out to be relatively simple, efficient, and robust. Due to its robustness, this staggered scheme has been used in many Computational Fluid Dynamics softwares; like Swash, Tunami, Comcot, Coulwave, Openfoam, Fluent, etc. Here, numerical models will be gradually built and refined, so that dedicated students will be able to compose their own codes; starting from the simplest wave simulation, up to the quite advanced one. We hope that you will learn various dynamical processes in fluids along with numerical methods use to simulate them.

\subsection{The leapfrog method}

We start with the formulation of leapfrog method for the linear SWE, and stress its equivalence with the finite volume method on a staggered grid. This scheme forms a solid building block for further development, because it is explicit, non-dissipative, and conditionally stable. Consider a governing equation for relatively small amplitude gravity waves above flat bottom $d_{0}$

$$
\begin{aligned}
\eta_{t}+d_{0} u_{x} & =0, \\
u_{t}+g \eta_{x} & =0,
\end{aligned}
$$

with $\eta(x, t)$ denoting the surface elevation measured from the undisturbed water level, and $u(x, t)$ the horizontal component of water particle velocity. In a spatial domain $0<x<L$ with hard wall left and right boundary 
conditions $u(0, t)=u(L, t)=0$, we define a staggered numerical grid with partition points $x_{\frac{1}{2}}=0, x_{1}, \cdots, x_{j-\frac{1}{2}}, x_{i}, x_{j+\frac{1}{2}}, \cdots, x_{N x+\frac{1}{2}}=L$.
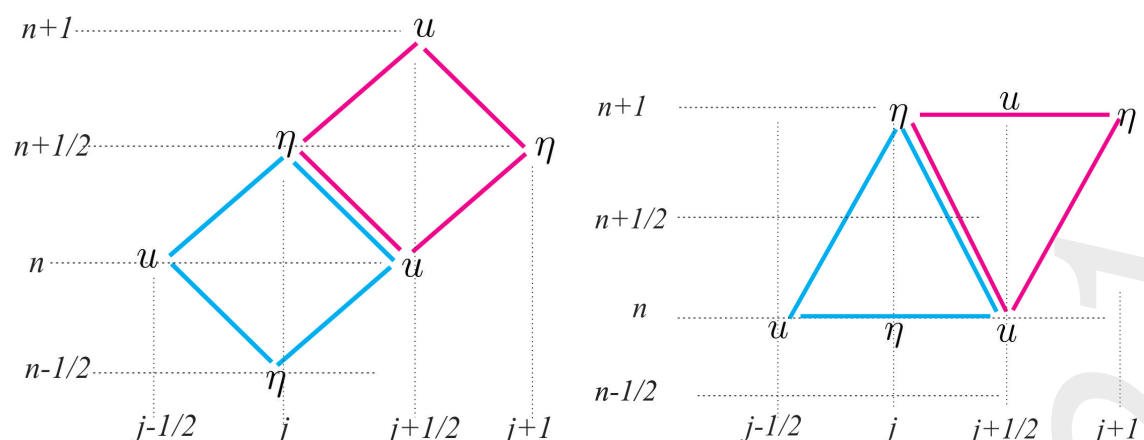

$n-1 / 2$
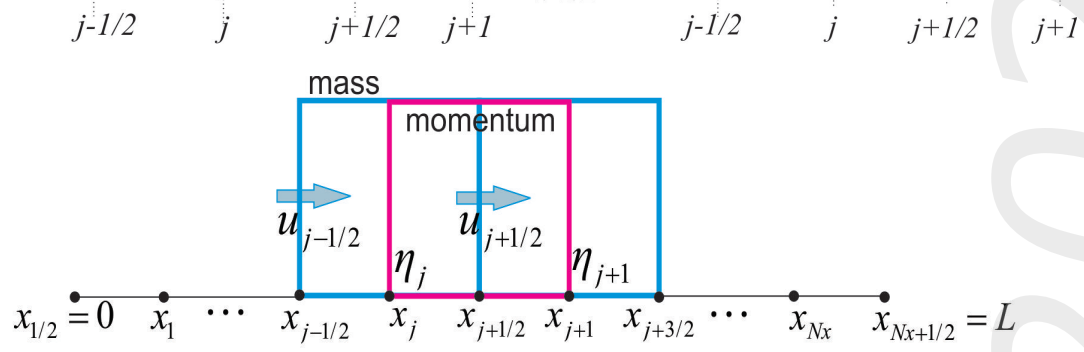

Figure 7.1.1: (Top left and right) Stencils of the original leapfrog scheme and its equivalent. (Bottom) Illustration of staggered grid, with a cell for mass and a cell for momentum.

We apply the second order leapfrog scheme to (7.1.1) around grid points $\left(x_{i}, t_{n}\right)$, and to (7.1.2) around $\left(x_{j+\frac{1}{2}}, t_{n+\frac{1}{2}}\right)$, with stencils as depicted in Figure 7.1.1 (Top left). They read

$$
\begin{aligned}
& \frac{\eta_{j}^{n+\frac{1}{2}}-\eta_{j}^{n-\frac{1}{2}}}{\Delta t}+d_{0} \frac{u_{j+\frac{1}{2}}^{n}-u_{j-\frac{1}{2}}^{n}}{\Delta x}=0, \\
& \frac{u_{j+\frac{1}{2}}^{n+1}-u_{j+\frac{1}{2}}^{n}}{\Delta t}+g \frac{\eta_{j+1}^{n+\frac{1}{2}}-\eta_{i}^{n+\frac{1}{2}}}{\Delta x}=0 .
\end{aligned}
$$

In $(7.1 .3,7.1 .4), \eta_{j}$ only appear at the staggered time grid $t_{n+\frac{1}{2}}$, whereas $u_{j+\frac{1}{2}}$ at the full time grid $t^{n}$, therefore we can as well write the equivalent formulas below

$$
\begin{gathered}
\frac{\eta_{i}^{n+1}-\eta_{i}^{n}}{\Delta t}+d_{0} \frac{u_{j+\frac{1}{2}}^{n}-u_{j-\frac{1}{2}}^{n}}{\Delta x}=0, \\
\frac{u_{j+\frac{1}{2}}^{n+1}-u_{j+\frac{1}{2}}^{n}}{\Delta t}+g \frac{\eta_{j+1}^{n+1}-\eta_{i}^{n+1}}{\Delta x}=0,
\end{gathered}
$$


with stencil shown in Figure 7.1.1 (Top right). Equations (7.1.5, 7.1.6) can be interpreted as the finite volume approximation of the linear SWE (7.1.1, 7.1.2), and will be coded and used extensively in further discussion. In a programming language, the staggered index in the $u$ variable must be shifted; we usually use $u_{j+1}$ for $u_{j+\frac{1}{2}}, j=0,1, \cdots, N_{x}$, see the pseudocode below.

Consider an interval $0<x<L$ with a staggered grid as depicted in Figure 7.1.1 (Bottom). Discrete version of mass conservation (7.1.1) in cell $\left[x_{j-\frac{1}{2}}, x_{j+\frac{1}{2}}\right]$ is just (7.1.5). This is because during time $\Delta t$ the change of mass in that cell depends on the difference between mass flux at sides $x_{j-\frac{1}{2}}$ and $x_{j+\frac{1}{2}}$. Neglecting the surface elevation $\eta$, mass flux at sides $x_{j-\frac{1}{2}}$ and $x_{j+\frac{1}{2}}$ is just $d_{0} u_{j-\frac{1}{2}}$ and $d_{0} u_{j+\frac{1}{2}}$, respectively. In Figure (7.1.1) (Bottom), we illustrate the mass cell $\left[x_{j-\frac{1}{2}}, x_{j+\frac{1}{2}}\right]$ centered at full grid point $x_{i}$, where we approximate the mass conservation (7.1.1). At the adjacent cell $\left[x_{i}, x_{j+1}\right]$ centered at staggered grid point $x_{j+\frac{1}{2}}$, we approximate (7.1.2). Consider (7.1.6), surface height difference between sides $x_{i}$ and $x_{j+1}$ of the cell $\left[x_{j}, x_{j+1}\right]$ with length $\Delta x$ will yield a hydrostatic pressure difference $g\left(\eta_{j+1}^{n+1}-\eta_{i}^{n+1}\right)$. This gravity force will induce a horizontal flow in that cell with rate $\left(u_{j+\frac{1}{2}}^{n+1}-u_{j+\frac{1}{2}}^{n}\right) / \Delta t$.

Further, as an equivalent form of leapfrog, the scheme $(7.1 .5,7.1 .6)$ is of second order accuracy. The stability condition is

$$
0 \leq C \leq 1, \quad \text { with the Courant number } C \equiv \sqrt{g d_{0}} \frac{\Delta t}{\Delta x} .
$$

This stability condition, known as the Courant-Frederic-Lewi (CFL) condition, can be obtained using the von Neumann method. The method starts by substituting the ansatz:

$$
\eta_{j}^{n}=r^{n} e^{i a j \Delta x}, \quad u_{j}^{n}=s^{n} e^{i a j \Delta x}
$$

into $(7.1 .5,7.1 .6)$, and after re-arrangement one obtained the following recursion formula

$$
\left(\begin{array}{l}
r^{n+1} \\
s^{n+1}
\end{array}\right)=A\left(\begin{array}{l}
r^{n} \\
s^{n}
\end{array}\right),
$$

with an amplification matrix $A$. The scheme is stable if the spectral radius of the matrix $A$ is less than one. 
Definition 7.1.1. For a square matrix $A$ with eigenvalues $\lambda_{1}, \cdots, \lambda_{n}$, spectral radius of a matrix $A$ denoted as $\rho(A)$ is

$$
\rho(A)=\max _{1 \leq i \leq n}\left|\lambda_{i}\right| .
$$

A numerical scheme is called non-dissipative if $\rho(A)=1$. A non-dissipative scheme is free from damping error.

Task: Prove the stability condition (7.1.7). Further, show that for any Courant number $C$ satisfying the stability condition $(7.1 .7)$, the scheme $(7.1 .5,7.1 .6)$ is non-dissipative.

Solution: Substituting the ansatz (7.1.8) into $(7.1 .5,7.1 .6)$ will yield

$$
\begin{aligned}
& \left(\begin{array}{ll}
1 & 0 \\
G & 1
\end{array}\right)\left(\begin{array}{l}
r^{n+1} \\
s^{n+1}
\end{array}\right)=\left(\begin{array}{ll}
1 & -D \\
0 & 1
\end{array}\right)\left(\begin{array}{l}
r^{n} \\
s^{n}
\end{array}\right), \\
& \text { with } D=d_{0} \frac{\Delta t}{\Delta x} 2 i \sin \xi, \quad G=g \frac{\Delta t}{\Delta x} 2 i \sin \xi, \quad \xi=\frac{a \Delta x}{2} .
\end{aligned}
$$

Re-arrangement will lead us to the recursion formula

$$
\left(\begin{array}{l}
r^{n+1} \\
s^{n+1}
\end{array}\right)=A\left(\begin{array}{l}
r^{n} \\
s^{n}
\end{array}\right), \quad \text { with } A=\left(\begin{array}{ll}
1 & -D \\
-G & 1+G D
\end{array}\right) .
$$

Quadratic equation for the eigenvalues of the matrix $A$ is

$$
(1-\lambda)^{2}-\lambda G D=\lambda^{2}+2\left(2 C^{2} \sin ^{2} \xi-1\right) \lambda+1=0 .
$$

In the case of real eigenvalues, at least one eigenvalue will have a norm larger than one, hence the scheme $(7.1 .5,7.1 .6)$ will be unconditionally unstable. The remaining possibility is the case of complex eigenvalues, which will happen if the discriminant is negative or

$$
4\left\{\left(2 C^{2} \sin ^{2} \xi\right)^{2}-4 C^{2} \sin ^{2} \xi+1\right\}-4 \leq 0
$$

or

$$
16 C^{2} \sin ^{2} \xi\left(C^{2} \sin ^{2} \xi-1\right) \leq 0 \quad \Leftrightarrow \quad 0 \leq C^{2} \sin ^{2} \xi \leq 1 .
$$

Furthermore, we can show that for this complex case, the norm of both eigenvalues is exactly one, which means that the scheme is free from damping error. For stability, the condition above should be satisfied for all $\xi \in \mathbb{R}$. Hence, stability condition for $(7.1 .5,7.1 .6)$ is $(7.1 .7)$.

q.e.d.

Since the linear SWE (7.1.1, 7.1.2) are models without damping, so having a non-dissipative scheme is important, and the leapfrog scheme (7.1.5, 
7.1.6) is indeed free from damping error. Furthermore, the scheme (7.1.5, 7.1.6) is explicit and therefore it is very simple and efficient. We note that for large gradient solutions, the scheme will suffer from weak dispersion error. Yet, this dispersion error will not appear for long waves.

\% Pseudo code: the staggered scheme for the linear SWE

$\%$ Arrangement of the unknowns on $[0, L]$ with grid staggered:

$\begin{array}{lllllllllllll}\% & u_{1} & h_{1} & u_{2} & h_{2} & \cdots & h_{j-1} & u_{j} & h_{j} & u_{j+1} & \cdots & h_{N x} & u_{N x+1}\end{array}$

$\%$ Notations $u_{j+1}$ here means $u_{j+\frac{1}{2}}, j=0,1, \cdots, N_{x}$ in $(7.1 .5,7.1 .6)$ $\%$

$\%$ Initialization

$\%$ Determine $\mathrm{e} 0(:)$ and $\mathrm{u} 0(:)$ from the initial conditions

$\%$ In the following we compute e1(:) and u1(:) for every time step for $\mathrm{n}=1: \mathrm{Nt}$

for $\mathrm{j}=1: \mathrm{Nx} \%$ One loop for computing $e 1(1: N x)$

$e 1(j)=e 0(j)-d^{*} d t / d x^{*}(u 0(j+1)-u 0(j))$;

end

u1(1) $=0 ; \%$ hard wall left boundary $u(0, t)=0$

for $\mathrm{j}=2: \mathrm{Nx} \%$ One loop for computing $u 1(2: N x)$

$\mathrm{u} 1(\mathrm{j})=\mathrm{u} 0(\mathrm{j})-\mathrm{g}^{*} \mathrm{dt} / \mathrm{dx} \mathrm{x}^{*}(\mathrm{e} 1(\mathrm{j})-\mathrm{e} 1(\mathrm{j}-1))$;

end

$\mathrm{u} 1(\mathrm{Nx}+1)=0 ; \%$ hard wall right boundary $u(L, t)=0$

$\%$ Plot the result

figure(1)

plot(xplot,e1(:)); \% or plot(xplot,u1(:))

$\%$ Update the values of $e 0$ and $u 0$

$\mathrm{e} 0(:)=\mathrm{e} 1(:)$;

$\mathrm{u} 0(:)=\mathrm{u} 1(:)$;

end

\section{Exercise 7.1.2.}

1. Derive the stability condition of the leapfrog method (7.1.5, 7.1.6). Show that this scheme is non-dissipative.

2. (Standing wave) Consider a basin, length $L=20 \mathrm{~m}$, depth $d_{0}=5 \mathrm{~m}$, with a hard wall boundary on the left and right at $x=0, x=L$. Take an initial condition $\eta(x, 0)=\cos (\pi x / L)$. Implement the leapfrog scheme, and simulate the back and forth motion of surface in a form of standing wave.

(a) Observe that this motion will continue for infinitely long period of time, without any real damping. Record wave signal at the 
position of antinode, i.e. $\eta(L, t)$. Please check if it moves with a constant amplitude for a long observation time. On the other hand, make sure that $\eta(L / 2, t)$ stays zero at the position of a node.

(b) Find the period and frequency of the calculated motion. Make sure that the calculated frequency confirm the normal modes frequency $\omega_{n}=\pi c_{0} / L$, with $c_{0}$ is the phase velocity $c_{0}^{2}=g d_{0}$.

(c) Apply the separation of variables, and derive the normal modes frequency of this closed basin system

$$
\omega_{n}=\sqrt{g d_{0}} \frac{n \pi}{L}, \quad n=1,2, \cdots
$$

Into a still water level in a closed basin, introduce a harmonic external force $f(t)$ with certain frequency $\omega$ that will induce resonance. Simulate a resonance phenomena in a closed basin. (Hint: you may put the external force as a surface disturbance $\eta(0, t)$.

3. (Periodic boundary) In several applications, sometimes we need to employ a perodic boundary condition $u(0, t)=u(L, t), \eta(0, t)=$ $\eta(L, t)$. Propose a suitable staggered discrete formula for this periodic boundary condition, in which what goes out of the right boundary will soon re-enter through the left boundary. Implement your periodic boundary formula to the linear SWE scheme, and simulate the right running monochromatic wave of a constant amplitude, circling around on a spatial domain $0 \leq x \leq L$.

4. In a tidal basin, length $L=20 \mathrm{~m}$, depth $d_{0}=5 \mathrm{~m}$, the water is initially at rest $\eta(x, 0)=0, u(x, 0)=0$. From the left boundary $x=0$ a monochromatic wave $\eta(0, t)$ enters, whereas on the right $x=L$ there is a hard wall boundary.

(a) (Progressive wave) Perform a simulation of a monochromatic wave $\eta(0, t)=0.2 \sin \pi t$ enters from the left, propagates to the right, hits the hard wall, and produces a left running reflected wave. Superposition between right and left running waves results in a wave with double amplitude. The left running wave propagates further to the left. Notice the appearance of strange behavior soon after the reflected wave arrived at $x=0$ and interact with the wave influx. Discuss this behavior.

(b) (Infinite spin up time) Repeat your computation using $\eta(0, t)=$ $0.2 \cos \frac{2 \pi t}{T}$, with $T=80$. Plot $\eta(L, t)$ together with $\eta(0, t)$. Dif- 
ferent from (a), directly from the begining, the cosine-wave influx induces a discontinuity, and this discontinuity remains for a long period of time, see Figure 7.1.2 (left). Spin up time is the influential time for initial state to vanish in the solution. This case is an example of infinite spin up time: in a model without damping, the effect of initial state (discontinuity) will remain over infinitely long period of time.
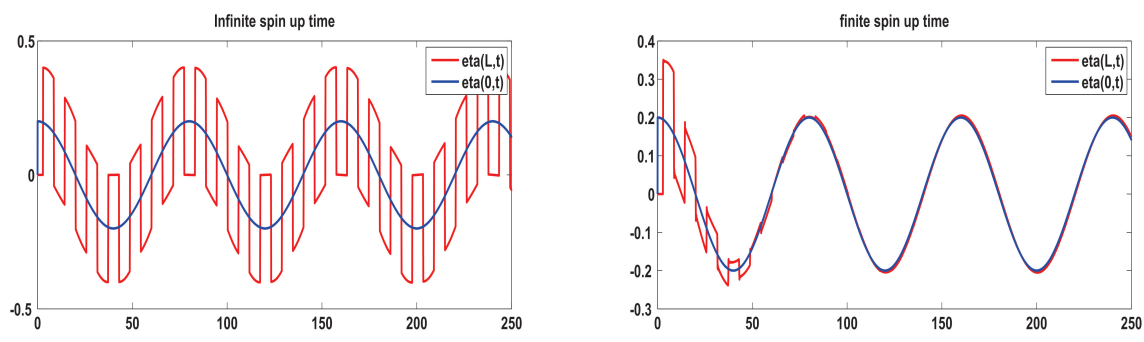

Figure 7.1.2: (Left) The discontinuity remains for a long period of time. (Right) The discontinuity decays after some time.

(c) Re-do exercise (b) but now using the model with linear friction

$$
\begin{aligned}
& \eta_{t}=-d_{0} u_{x} \\
& u_{t}=-g \eta_{x}-C_{f} u
\end{aligned}
$$

For computation, use the friction parameter $C_{f}=0.1$, and reproduce Figure 7.1.2 (right). Show that in this example, we have a finite spin up time. Explain your result.

Some terminology: a cold start is when a simulation is first initialized and then needs to be spin up. A hot start is a restart of a model from the saved results of a previous simulation, which can be used to eliminate or reduce the spin-up time.

\subsection{Conservative scheme for the half linear SWE}

Consider the full shallow water equation (SWE) written in conservative form as follows

$$
\begin{aligned}
h_{t}+(h u)_{x} & =0 \\
(h u)_{t}+\left(h u^{2}+\frac{1}{2} g h^{2}\right)_{x} & =g h d_{x}-C_{f}|u| u .
\end{aligned}
$$

where $h(x, t)$ denotes the total water height, $\eta(x, t)$ the surface elevation, $u(x, t)$ the horizontal fluid velocity, $d(x)$ the bottom topography, and $C_{f}$ 
the friction coefficient, see Fig. (7.2.1). Their commonly used equivalent forms are

$$
\begin{aligned}
\eta_{t}+(h u)_{x} & =0, \\
u_{t}+u u_{x}+g \eta_{x} & =-C_{f} \frac{|u| u}{h},
\end{aligned}
$$

that can be obtained through a relation for the advection term

$$
u u_{x}=\frac{1}{h}\left(\frac{\partial(q u)}{\partial x}-u \frac{\partial q}{\partial x}\right)
$$

with $q=h u$ denoting the horizontal momentum. Later, relation (7.2.5) will be used for approximating the advection term.

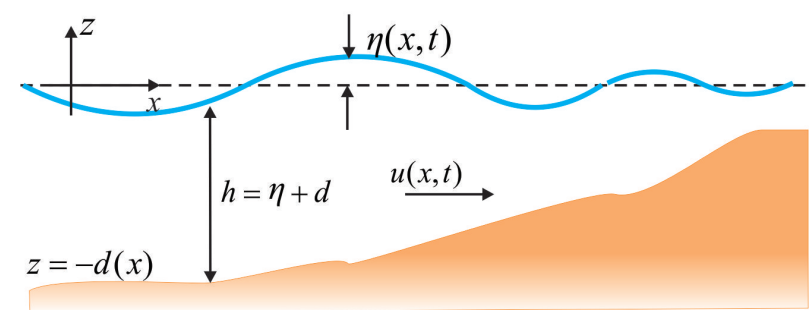

Figure 7.2.1: A sketch of fluid layer and notations.

We consider the half linear SWE consisting of the full mass conservation and linearized momentum balance, as follows

$$
\begin{aligned}
\eta_{t}+(h u)_{x} & =0, \\
u_{t}+g \eta_{x} & =0 .
\end{aligned}
$$

on a domain $[0, L]$ with a staggered partition as depicted in Figure 7.2.2.

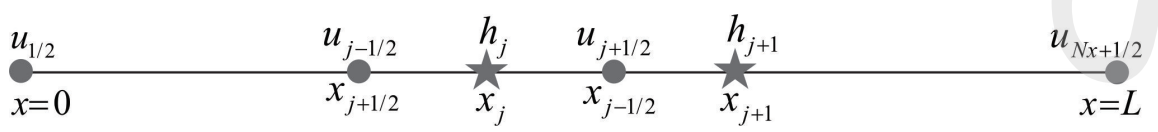

Figure 7.2.2: Staggered grid with configuration of calculated variables $h$ and $u$.

Let we denote $q \equiv h u$. Approximation for mass conservation (7.2.6) at cell $\left[x_{j-\frac{1}{2}}, x_{j+\frac{1}{2}}\right]$ is

$$
\frac{\eta_{j}^{n+1}-\eta_{j}^{n}}{d t}+\frac{q_{j+\frac{1}{2}}^{n}-q_{j-\frac{1}{2}}^{n}}{\Delta x}=0,
$$


with

$$
\left.q\right|_{j+\frac{1}{2}}=u_{j+\frac{1}{2}} * h_{j+\frac{1}{2}} .
$$

In (7.2.9) values of $h$ is indicated with ${ }^{*}$ because at points numbered by $j+\frac{1}{2}$ there is no value of $h$, and it should be approximated. The first order approximation is by upwind, as follows

$$
{ }^{*} h_{j+\frac{1}{2}}= \begin{cases}h_{j}, & \text { for } u_{j+\frac{1}{2}}>0 \\ h_{j+1}, & \text { for } u_{j+\frac{1}{2}}<0\end{cases}
$$

which is a direct consequence of considering flow direction in the approximation.

We note here, the term $\left.q\right|_{j+\frac{1}{2}}={ }^{*} h_{j+\frac{1}{2}} u_{j+\frac{1}{2}}$ expresses the first order approximation of mass flux at edge $x_{j+\frac{1}{2}}$. When the flow is going to the right $u_{j+\frac{1}{2}} \geq 0$, we take the left flux $h_{j} u_{j+\frac{1}{2}}$, and when the flow is going to the left $u_{j+\frac{1}{2}}<0$, we take the right flux $h_{j+1}^{2} u_{j+\frac{1}{2}}$. Hence approximation (7.2.8) preserves mass conservation property at every cell $\left[x_{j-\frac{1}{2}}, x_{j+\frac{1}{2}}\right]$, and this holds for either flow direction.

Alternative approximation for mass (7.2.6) as given in Jochen is as follows

$$
\frac{\eta_{j}^{n+1}-\eta_{j}^{n}}{\Delta t}+\frac{1}{\Delta x}\left(u_{j+\frac{1}{2}}^{+} h_{j}^{n}+u_{j+\frac{1}{2}}^{-} h_{j+1}^{n}-u_{j-\frac{1}{2}}^{+} h_{j-1}^{n}-u_{j-\frac{1}{2}}^{-} h_{j}^{n}\right)=0,
$$

where

$$
u_{j+\frac{1}{2}}^{+}=\frac{1}{2}\left(u_{j+\frac{1}{2}}^{n}+\left|u_{j+\frac{1}{2}}^{n}\right|\right), \quad u_{j+\frac{1}{2}}^{-}=\frac{1}{2}\left(u_{j+\frac{1}{2}}^{n}-\left|u_{j+\frac{1}{2}}^{n}\right|\right) .
$$

Task: Show that (7.2.8) and (7.2.11) are equivalent.

$\%$ Pseudo code: the modified staggered scheme for the half-linear SWE

$\%$ Arrangement of the unknowns on $[0, L]$ with grid staggered:

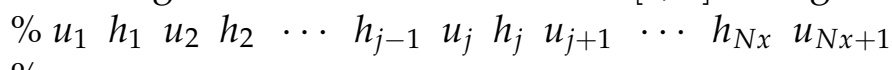
$\%$

$\%$ The loop for computing e1(:) is replaced with for $\mathrm{j}=2 \mathrm{Nx}$ if $(\mathrm{u} 0(\mathrm{j})>0)$

$h(j)=e 0(j-1)+d(j-1) ; \%$ case $u 0(j)>0$ adopt the left info else 


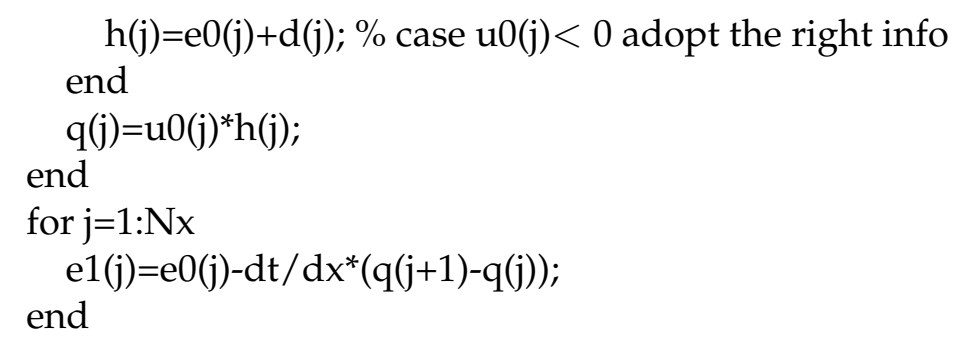

(Mass conservation scheme for varying bottom) Consider the following linear SWE with varying bottom

$$
\begin{aligned}
& \eta_{t}+(d(x) u)_{x}=0, \\
& u_{t}+g \eta_{x}=0,
\end{aligned}
$$

In order to keep the mass conserve in this simulation, you have to implement the following

$$
\eta_{j}^{n+1}=\eta_{j}^{n}-\frac{\Delta t}{\Delta x}\left({ }^{*} d_{j+\frac{1}{2}}^{n} u_{j+\frac{1}{2}}^{n}-{ }^{*} d_{j-\frac{1}{2}}^{n} u_{j-\frac{1}{2}}^{n}\right)
$$

with ${ }^{*} d_{j+\frac{1}{2}}^{n}$ is calculated using upwind

$$
* d_{j+\frac{1}{2}}^{n}= \begin{cases}d_{j,}^{n}, & \text { if } u_{j+\frac{1}{2}}^{n}>0 \\ d_{j+1}^{n}, & \text { if } u_{j+\frac{1}{2}}^{n}<0\end{cases}
$$

\section{Exercise 7.2.1.}

1. Consider $(7.2 .12,7.2 .13)$ for $0<x<L$, and $t>0$, with absorbing boundaries at the two ends. Adopt a piecewise constant bathymetri.

$$
d(x)= \begin{cases}d_{1}, & x<L / 2 \\ d_{2,} & x>L / 2\end{cases}
$$

Take an initial condition $\eta(x, 0)=$ a hump, with zero initial velocity. As time evolves, this hump will split into two according to d'Alembert. Put the hump, such that the left running wave propagates through $x=0$, whereas the right running wave propagates over a bottom step. Your simulation results will look like Figure 7.2.3. Make sure that in the bottom step-downwards i.e. $d_{2}>d_{1}$, transmitted wave has the same phase as the incident wave but reflected wave has negative phase. Whereas in the bottom step-upwards, i.e. $d_{2}<d_{1}$, both transmitted and reflected waves have the same phase as the incident wave. Check the total mass during computation by calculating $M(t)=\int_{0}^{L} h(x, t) d x$. 

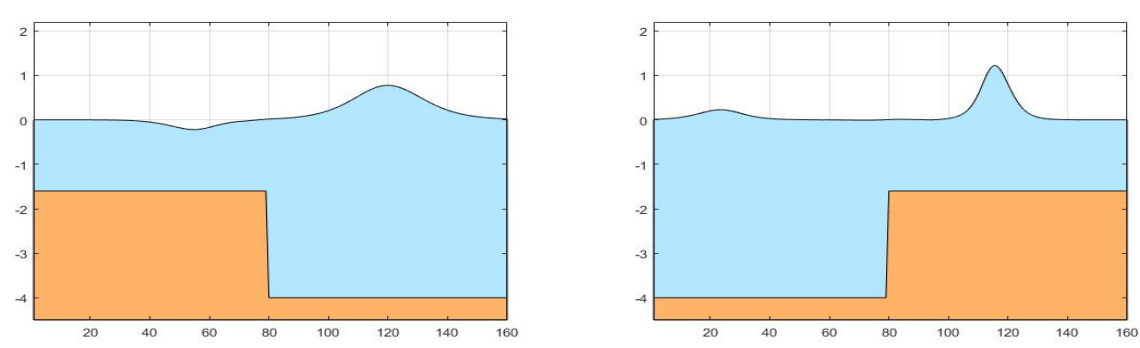

Figure 7.2.3: Transmitted and reflected waves produce by a wave propagates over a bottom step-downwards (left) and a bottom step-upwards (right).

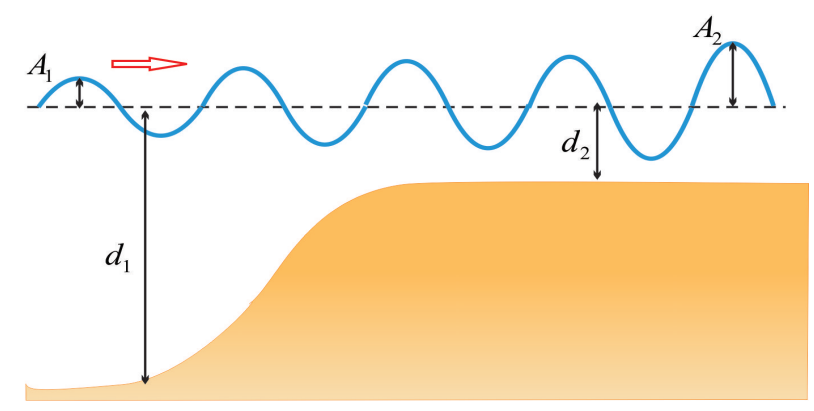

Figure 7.2.4: Shoaling effect on waves propagating over a decreasing depth.

2. Consider a bottom topography in the form of a shelf

$$
d(x)= \begin{cases}d_{1}, & \text { for } x<0 \\ \text { a smooth transition, } & \text { for } 0<x<L \\ d_{2}, & \text { for } x>L\end{cases}
$$

with $d_{2}<d_{1}$. Simulate the wave shoaling phenomena: wave amplitude increases as depth decreases. Show that your numerical simulation confirm the wave shoaling coefficient formula $\frac{A_{2}}{A_{1}}=\left(\frac{d_{1}}{d_{2}}\right)^{1 / 4}$. We often observe this shoaling phenomena while looking at waves on beaches. 


\subsection{The full nonlinear SWE}

Consider the full nonlinear SWE.

$$
\begin{aligned}
& \eta_{t}+(h u)_{x}=0, \\
& u_{t}+u u_{x}+g \eta_{x}=0,
\end{aligned}
$$

with $h=\eta+d(x)$ denotes water thickness.

On the staggered grid, the momentum equation (7.3.2) is approximated at cell $\left[x_{j-1}, x_{j}\right]$, or explicitly

$$
\frac{d u_{j-\frac{1}{2}}^{n}}{d t}+g \frac{\eta_{j}^{n+1}-\eta_{j-1}^{n+1}}{\Delta x}+\left(u u_{x}\right)_{j-\frac{1}{2}}^{n}=-\left.C_{f} \frac{|u| u}{h}\right|_{j-\frac{1}{2}} ^{n} .
$$

The nonlinear friction term is calculated using Picard linearization

$$
(|u| u)^{n} \approx\left|u^{n}\right| u^{n+1} .
$$

The following are three options to approximate the advection term $u u_{x}$.

A. Central difference

$$
\left.u u_{x}\right|_{j-\frac{1}{2}} ^{n} \approx u_{j-\frac{1}{2}}^{n} \frac{u_{j+\frac{1}{2}}^{n}-u_{j-\frac{3}{2}}^{n}}{2 \Delta x}
$$

B. First order upwind method

$$
\left.u u_{x}\right|_{j-\frac{1}{2}} ^{n} \approx \begin{cases}u_{j-\frac{1}{2}}^{n} \frac{u_{j-\frac{1}{2}}^{n}-u_{j-\frac{3}{2}}^{n}}{\Delta x}, & \text { if } u_{j-\frac{1}{2}}^{n}>0 \\ u_{j-\frac{1}{2}}^{n} \frac{u_{j+\frac{1}{2}}^{n}-u_{j-\frac{1}{2}}^{n}}{\Delta x}, & \text { is } u_{j-\frac{1}{2}}^{n}<0\end{cases}
$$

C. Energy conservative, which is based on the fact that $u u_{x}=\left(\frac{1}{2} u^{2}\right)_{x}$

$$
\left.u u_{x}\right|_{j-\frac{1}{2}} ^{n} \approx \frac{1}{2}\left(\left(* u_{j}^{n}\right)^{2}-\left({ }^{*} u_{j-1}^{n}\right)^{2}\right) / \Delta x .
$$

\subsubsection{Wet-dry procedures}

For flood simulation or any other simulations that involve dry areas, a slight modification is needed. In our setting, grid points extend over the whole domain, including dry areas. In order to implement (7.3.3) we need to make sure that the water thickness ${ }^{*} h_{j-\frac{1}{2}}>0$. Therefore, during simulation $u_{j-\frac{1}{2}}$ is computed using (7.3.3) only when the cell $\left[x_{j-1}, x_{j}\right]$ 
is 'wet', namely when the total water thickness is positive, to be precise ${ }^{*} h_{j-\frac{1}{2}}$ should be larger than a threshold number. This treatment is called the wet-dry procedure.

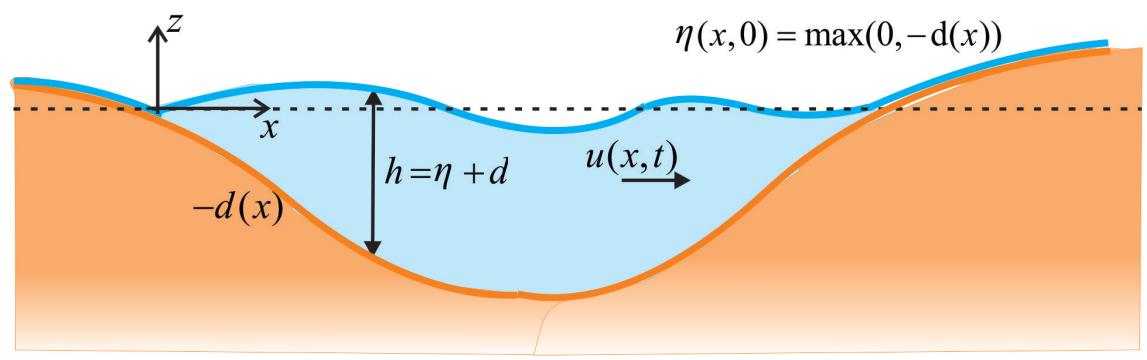

Figure 7.3.1: In a computation that involves dry areas the still water level is modified to $\eta(x, 0)=\max (0,-d(x))$.

Another caution is on setting the initial still water level. Normally, we just take $\eta(x, 0)=0$ and $u(x, 0)=0$, implementing this will cause $h(x, 0)=$ $\eta(x, 0)+d(x)$ to be negative on dry areas. This is because the bathymetry $d(x)$ refers to (positive) water depth in 'wet' regions and (negative) land elevation in 'dry' regions of the domain, see Figure 7.3.1. To prevent instability due to negative $h$, the initial still water level should be modified to $\eta(x, 0)=\max (0,-d(x))$. In literature, this modification is called 'thin layer technique', since we interpret $\max (0,-d(x)+\varepsilon)$ as putting an infinitely thin layer with thickness $\varepsilon$ on 'dry' region.

\subsubsection{Shapiro filter}

The leapfrog scheme employed here suffers from numerical dispersion error, i.e. oscillations developing on wavelengths of $2 \Delta x$. If oscillations arise due to numerical errors, for better presentation results we sometimes want to remove them. We can remove these small-scale oscillations by employing the following first-order Shapiro filter (Shapiro, 1970). Assume from a numerical scheme, we calculated $\eta_{j}^{n}$ that contains small wiggles, before plotting $\eta_{j}^{n}$ we smudge it first by calculating $\eta_{j}^{*}$ using the Shapiro's formula below

$$
\eta_{j}^{*}=(1-\varepsilon) \eta_{j}^{n}+\frac{1}{2} \varepsilon\left(\eta_{j+1}^{n}+\eta_{j-1}^{n}\right),
$$

with $\varepsilon$ is a smoothing parameter, that can be taken any small number below one. Then, instead of plotting $\eta_{j}^{n}$, we plot $\eta_{j}^{*}$ which is smoother. Note that we keep using $\eta_{j}^{n}$ for calculating the value of $\eta_{j}^{n+1}$ in the next time 
step, so the role of $\eta_{j}^{*}$ here is really just for plotting.

Task: Relates (7.3.5) with the central difference scheme of diffusion equation $\eta_{t}=k \eta_{x x}$. Next, explain that (7.3.5) is indeed 'a smoothing' procedure.

\section{Exercise 7.3.1.}

The full SWE and wet-dry procedure

1. Simulate wave shoaling phenomena in the previous exercise, but now using the half nonlinear SWE, i.e. continuity nonlinear, momentum linear. Interpret your result.

2. For a computational domain $0<x<L$ simulate the motion of a standing wave with initial condition $\cos \pi x / L$ using the full nonlinear SWE.

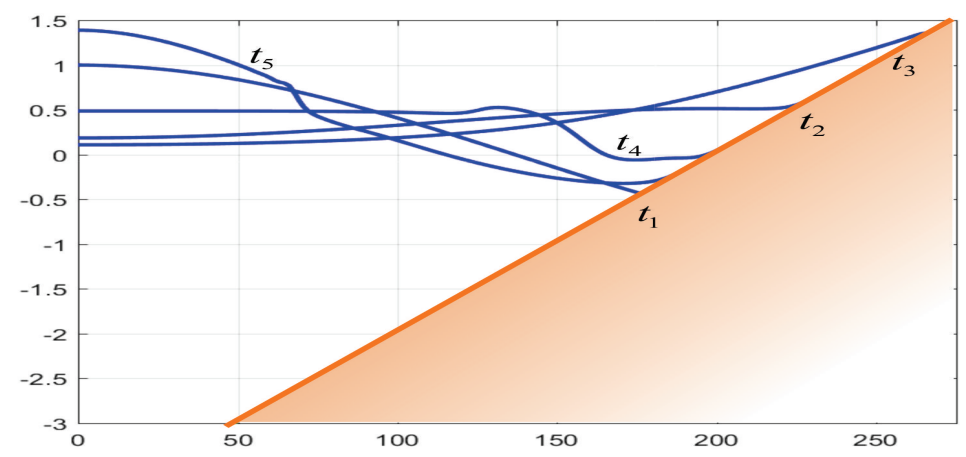

Figure 7.3.2: Plot of surface wave uprush on a sloping beach at subsequent times.

3. Conduct a simulation of wave running up and down on a sloping beach. Simulation result as shown in Figure 7.3.2 uses a monochromatic initial condition $\eta(x, 0)=\max (\cos (\pi x / L),-d(x))$, with $d(x)$ is the sloping beach with dry area.

4. Test your scheme for simulating the ultimate test 1 in Jochen page 80 , or the ultimate test 2 page 82 . Use your scheme to simulate your own numerical flow configuration.

\section{Exercise 7.3.2.}

Wet-dry procedure and momentum conservative scheme 
1. (Dam break wet bed case) Use the full nonlinear SWE to simulate dam break problem on a flat bottom topography $z=0$. Under the following initial condition

$$
h(x, 0)= \begin{cases}h_{L}, & \text { untuk } x<x_{0} \\ h_{R}, & \text { untuk } x>x_{0},\end{cases}
$$

with $h_{L} \neq h_{R}$, and both $h_{L}, h_{R}$ are positive, the case is considered as dam break on the wet bed case. As time progresses, water height evolves into a wave with steep front, see Figure 7.3.3 (Left).
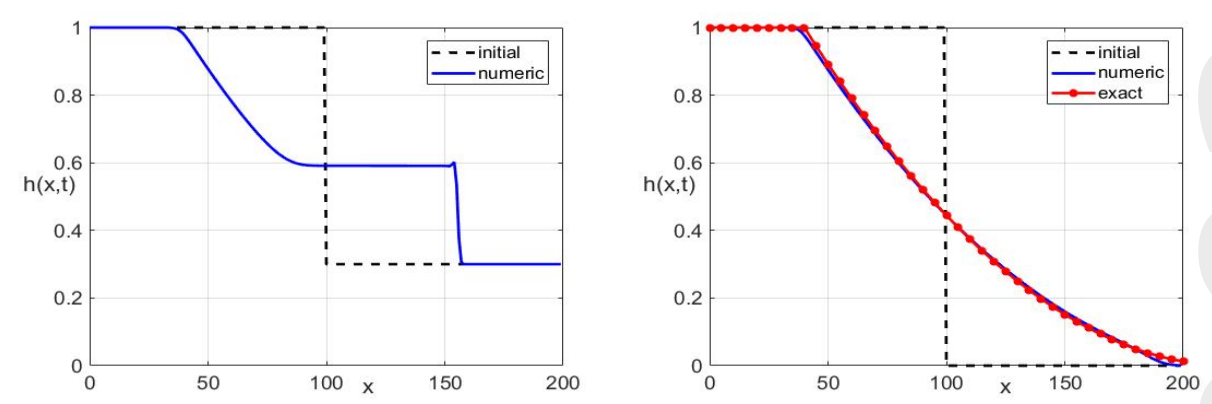

Figure 7.3.3: Surface profiles in dam break simulations; (left) the wet-bed case, (right) the dry-bed case.

2. (Dam break dry bed case) Similar like above, but now use

$$
h(x, 0)=\left\{\begin{array}{ll}
h_{0}, & \text { untuk } x<x_{0} \\
0, & \text { untuk } x>x_{0},
\end{array} \quad \text { with } h_{0}>0 .\right.
$$

The correct simulation of dam break dry bed case should produce a rarefaction wave as shown in Figure 7.3.3 (right).

Note that analytical solution of the dam break dry bed case is follows

$$
\begin{aligned}
& h(x, t)= \begin{cases}h_{0}, & \left(x-x_{0}\right) / t<-c_{0} \\
h_{0}\left(\frac{2}{3}-\frac{x-x_{0}}{3 t c_{0}}\right)^{2}, & -c_{0}<\left(x-x_{0}\right) / t<2 c_{0} \\
0, & \left(x-x_{0}\right) / t>2 c_{0}\end{cases} \\
& u(x, t)= \begin{cases}\frac{2}{3} c_{0}\left(1+\frac{x-x_{0}}{t c_{0}}\right), & -c_{0}<\left(x-x_{0}\right) / t<2 c_{0} \\
0, & \text { elsewhere, }\end{cases}
\end{aligned}
$$

with $c_{0}=\sqrt{g h_{0}}$. Water height profile of this dam break dry bed solution is different than the dam break wet bed solution. In this dry 
bed case, there is no steep front, instead it evolves as a rarefaction wave, see Figure 7.3.3 (right).

Experience that all three numerical approximation of the advection term $u u_{x}$ will produce false results, i.e. the wave has a steep front! The correct result can be achieved if we implement the momentum conservative scheme by Stelling \& Duijnmeijer [13]. This dam break dry bed case is in fact an important bench mark test for the correct SWE solver.

3. Repeat the above exercise, but now incorporates a friction term $C_{f} u|u| / h$ in SWE model. Apply the Picard linearization to approximate the friction term, and simulate the dam break dry bed case with friction coefficient $C_{f}=0.1$. Interpret your result.

\subsection{Staggered finite volume method for 2D SWE}

In this section, we will discuss the staggered finite volume for two dimensional shallow water equations. The method discussed here will be a direct generalization of the staggered scheme of the 1-dimensional SWE.

Consider the following 2-dimensional shallow water equations, hold in a rectangular domain $0<x<L, 0<y<M$,

$$
\begin{aligned}
h_{t}+(h u)_{x}+(h v)_{y} & =0 \\
u_{t}+u u_{x}+v u_{y}+g \eta_{x} & =0 \\
v_{t}+u v_{x}+v v_{y}+g \eta_{y} & =0 .
\end{aligned}
$$

The linearized equations for flat bottom $d(x)=d_{0}$ are

$$
\begin{aligned}
h_{t}+d_{0}\left(u_{x}+v_{y}\right) & =0 \\
u_{t}+g \eta_{x} & =0 \\
v_{t}+g \eta_{y} & =0 .
\end{aligned}
$$

Consider a computational domain $[0, L] \times[0, M]$ with a staggered grid partition sketches in Figure 7.4.1. This staggered grid is known as the Arakawa-C grid. For the first simulation, we consider a layer of homogeneous fluid in a rectangular basin. For simulation, we implement the linear SWE over a flat bottom $d_{0}$ with hardwall boundaries along the four sides. 


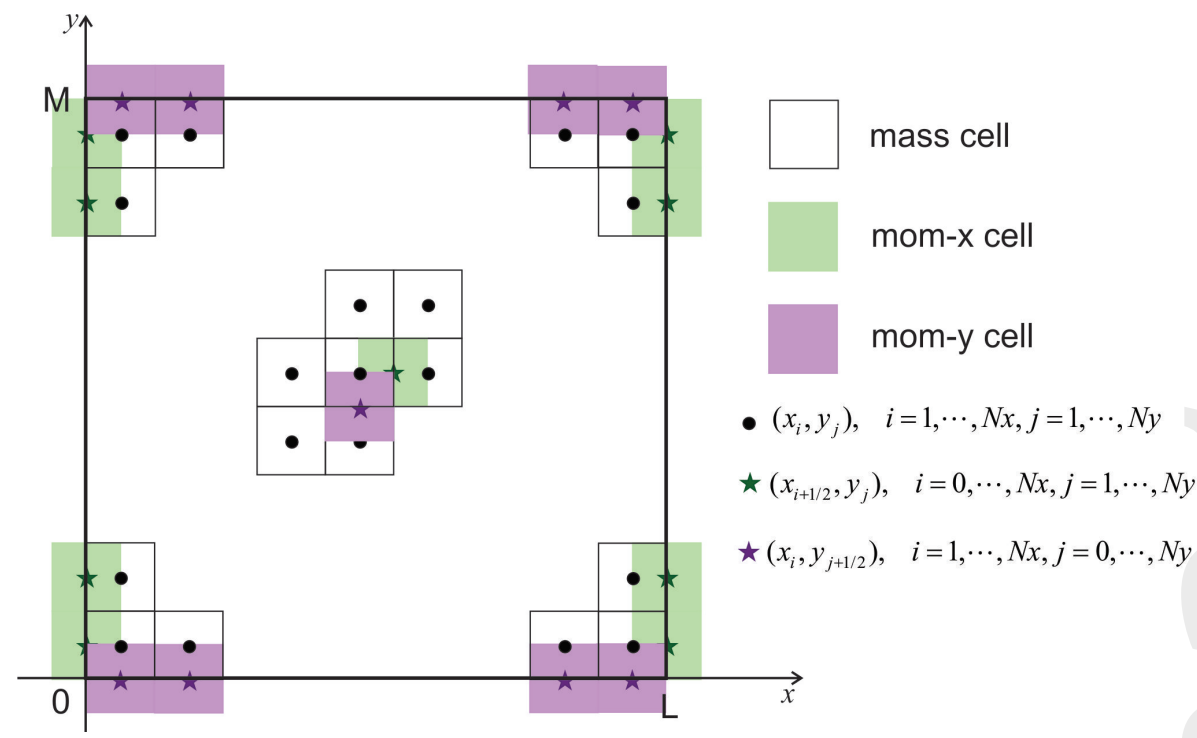

Figure 7.4.1: Arakawa-C grid with cells for mass, momentum-x, and momentum- $y$. Hard-wall boundaries are applied along the four sides.

Task: Formulate these four hardwall boundaries.

Formulation of the 2-dimensional staggered grid is analogous to the staggered grid 1-dimensional scheme. The mass conservation (7.4.4) is discretized at the mass cell, which is the white square centered at $\left(x_{i}, y_{j}\right)$, it reads

$$
\begin{array}{r}
\frac{h_{i, j}^{n+1}-h_{i, j}^{n}}{\Delta t}+d_{0} \frac{u_{i+1 / 2, j}^{n}-u_{i-1 / 2, j}^{n}}{\Delta x}+d_{0} \frac{v_{i, j+1 / 2}^{n}-v_{i, j-1 / 2}^{n}}{\Delta y}=0, \\
\quad i=1,2, \cdots, N x, j=1,2, \cdots, N y .
\end{array}
$$

Momentum balance (7.4.5) is approximated at the x-momentum cell, the light grey square centered at $\left(x_{i+1 / 2}, y_{j}\right)$

$$
\begin{gathered}
\frac{u_{i+1 / 2, j}^{n+1}-u_{i+1 / 2, j}^{n}}{\Delta t}+g \frac{\eta_{i+1, j}^{n+1}-\eta_{i, j}^{n+1}}{\Delta x}=0, \\
\quad i=1,2, \cdots, N x-1, j=1,2, \cdots, N y
\end{gathered}
$$

and (7.4.6) is approximated at the y-momentum cell, the dark grey square 
centered at $\left(x_{i}, y_{j+1 / 2}\right)$

$$
\begin{gathered}
\frac{v_{i, j+1 / 2}^{n+1}-v_{i, j+1 / 2}^{n}}{\Delta t}+g \frac{\eta_{i, j+1}^{n+1}-\eta_{i, j}^{n+1}}{\Delta y}=0, \\
\quad i=1,2, \cdots, N x, j=1,2, \cdots, N y-1
\end{gathered}
$$

Note that (7.4.7) is the exact formulation of a discrete mass conservation at the mass cell.

Task: Show that the CFL condition for this scheme is

$$
\Delta t \leq \frac{\min (\Delta x, \Delta y)}{\sqrt{2 g d_{0}}} .
$$

\section{Exercise 7.4.1.}

1. Simulate the standing wave motion in this rectangular basin. Use three type of initial conditions:

(a) $\eta(x, y, 0)=\cos (\pi x / L)$,

(b) $\eta(x, y, 0)=\cos (\pi y / M)$,

(c) $\eta(x, y, 0)=\cos (\pi x / L) \cos (\pi y / M)$.

2. In a square lake of $500 \mathrm{~m} \times 500 \mathrm{~m}$, and $10 \mathrm{~m}$ depth, examine the evolution of an initial disturbance to its surroundings. Show that the wave will spread out in all directions in the shape of a circular wave, see Figure 7.4.2. Use grid spacings of $\Delta x=\Delta y=10 \mathrm{~m}$, and time step $\Delta t=0.1 \mathrm{~s}$ to meet the CFL stability. For your first simulation, use hard wall boundaries. Next, experiment with absorbing boundaries. Explain why $u\left(0, y, t_{n+1}\right)=u\left(\Delta x, y, t_{n+1}\right)$ produces an absorbing left boundary. Is it possible to achieve ideal absorbing boundaries? ${ }^{1}$
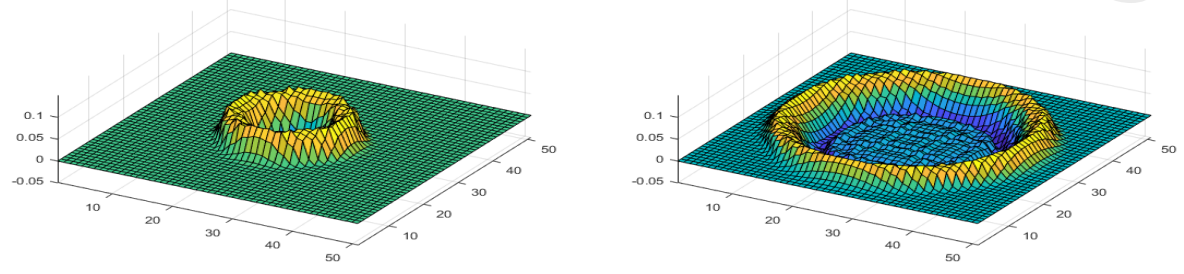

Figure 7.4.2: Circular waves resulting from a point-source disturbance.

\footnotetext{
${ }^{1}$ The study of absorbing boundary conditions for the 2D nonlinear SWE is still ongoing.
} 
3. (Wave interaction with an obstacle) Create a topography with a smooth obstacle as shown in Figure 7.4.3 (left). One simplest way to do that is to use a piecewise constant topography and then apply a 2D Shapiro filter to smudge bottom discontinuities. Then, select your 2D initial wave. Make sure that your obstacle emerges from the surface, so that your simulation contains dry area. As a result, the wet-dry procedure must be implemented throughout the simulation time. The snapshot shown in Figure 7.4.3 used an initial wave $\eta(x, y, 0)=\cos (\pi x / L)$, where $L$ represents the width of the rectangular basin, as well as closed lateral boundaries.
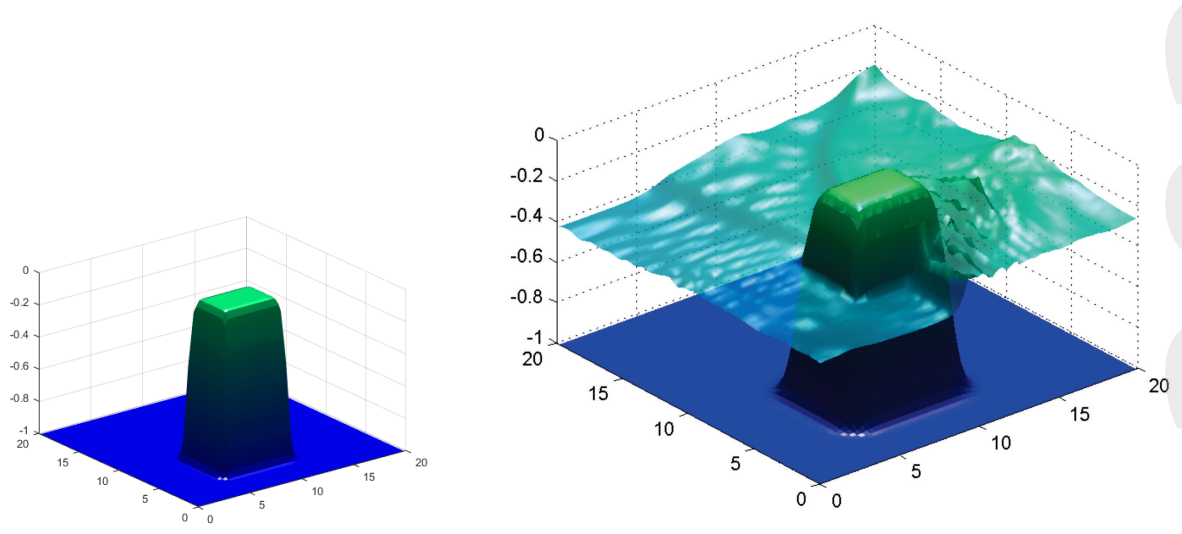

Figure 7.4.3: (Left) the obstacle, (right) waves interact with an obstacle. 


\section{Bibliography}

[1] Alexiades V., Solomon, A.D., Mathematical Modelling of Melting and Freezing Processes, Hemisphere Publishing Corporation, Washington DC, 1981.

[2] Durran, D., Numerical Methods for Fluid Dynamics, with applications to Geophysics, $2^{\text {nd }}$ ed., Springer, 2010.

[3] Ni, D., Traffic Flow Theory: Characteristics, Experimental Methods, and Numerical Techniques, Elsevier, 2015.

[4] Dean, R.A., Dalrymple R.A., Water wave mechanics for engineers and scientists, World Scientific, 2000.

[5] Haberman, R., Mathematical Models (Mechanical Vibrations, Population Dynamics, and Traffic Flow, SIAM, Texas, 1998.

[6] Hoffmann, J., Numerical Methods for Engineers and Scientists, Mc.Graw-Hill, 1992.

[7] Jochen Kämpf, Ocean Modelling for Beginners, Springer 2009.

[8] Lighthill, M.J., Whitham, G.B., On kinematic waves I: Flood movement in long rivers, The Royal Society: Proceedings A, 1955.

[9] Lighthill, M.J., Whitham, G.B., On kinematic waves II: A theory of traffic flow on long, crowded roads, The Royal Society: Proceedings A, 1955.

[10] Mattheij, R.M.M., Rienstra, S.W., ten Thije Boonkkamp, J.H.M., Partial Differential Equations, Modelling, Analysis, Computations, SIAM Monographs, Philadelphia, 2005

[11] Richards, P.I., Shock waves on the highway, Operation Research, 1956.

[12] Roberts, A.J., A one-dimensional introduction to Continuum Mechanics, World Scientific, 1994. 
[13] Stelling, G.S., Duinmeijer, S.P.A., A Staggered Conservative Scheme for Every Froude Number in Rapidly Varied Shallow Water Flows, Int. J. for Numer. Meth. Fluids, 43, (2003), 1329-1354.

[14] Stelling, G.S., OD box models, ODEs and systems of ODEs, lecture slides.

[15] Strauss, W., Partial Differential Equations, an Introduction, John Wiley \& Sons, 1992.

[16] Tarwidi, D., Pudjaprasetya, S. R., Godunov method for Stefan problems with enthalpy formulations. East Asian Journal on Applied Mathematics, 3(2), 107-119, 2013.

[17] Zijlema, M., Computational Modelling of Flow and Transport, Delft University of Technology, 2011-2012. 


\section{Index}

a cold start, 105

a hot start, 105

absorbing boundary, 94

accuracy, 12, 20

advection term, 110

amplification matrix, 101

Arakawa C-grid, 114

backward difference, 25

Backward time center space (BTCS), 35

blood flow, 91

Burgers' equation, 61

center difference, 25

CFL condition, 101, 116

characteristic gradient, 46,72

characteristic method, 45

circular wave, 116

conservation scheme, 105

conservative equation, 57

consistency, 13, 26, 27

convection, 45

convergence, 12, 13, 26

Courant number, 31, 49, 92

Crank-Nicolson, 21, 36

difference equation, 27

Dirichlet boundary, 29, 94

dispersion error, 55

dispersion relation, 88

enthalpy formulation, 39

Euler equation, 79

explicit, 12, 21, 29 finite difference, 12, 17, 25

finite volume method, 62,75

fixed ends, 94

forward difference, 25

Forward time center space (FTCS), 29,47

Godunov method, 65, 77

hard wall boundary, 94

harmonic oscillator, 17

implicit, 12, 21, 35

kinematic boundary condition, 80

kinematic LWR model, 69

Lax equivalence theorem, 27

Lax method, 91

Lax-Wendroff method, 93

leapfrog scheme, 55, 99

local discretization error, 26

mass conservation scheme, 108

midpoint rule, 12

modified differential equation, 26

momentum conservative scheme, 112

moving boundary, 37

Neumann boundary, 29, 94

non-dissipative, 102, 103

order of the scheme, 27

Rangkine Hugoniot condition, 58, 74

rarefaction wave, 59 
Riemann invariant form, 95

Riemann problem, 58

Shallow water equation, 81

Shapiro filter, 111

shoaling, 109

shock speed, 58,74

shock wave, 58,74

similarity solution, 58

spectral radius, 92, 101

spin up time, 105

stability, 12, 13, 18, 27, 31, 49, 92, 101

staggered grid, 99

standing wave, 89, 93

Stefan problem, 37

theta method, 36

Time integration scheme, 12

time integration scheme, 13

traffic flow, 68

trapezoidal, 12, 18, 21

trapezoidal method, 18

truncation error terms, 27

upwind method, 47,76

von Neumann method, 27, 31, 49, 101

wet-dry procedure, 110, 112 


\section{About the Author}

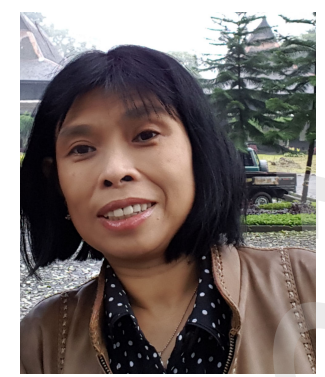

Sri Redjeki Pudjaprasetya is professor from Institut Teknologi Bandung, Indonesia. She obtained her bachelor and master degrees in Mathematics from Institut Teknologi Bandung in 1988 \& 1990. She received her Ph.D. degree in Applied Mathematics from the University of Twente, the Netherlands, in 1996. Since 1988 she has became a lecturer in Mathematics ITB, and joined in the Industrial and Financial Mathematics Research Group. Her field of research is about non-linear waves, with the application of water wave problems, including tsunami. She also conduct research on modeling and simulation of open channel flows, and its interaction with hydraulic structures. In recent years, she has conducted research in traffic flow problems. In addition to teaching mathematics for the first and second year students, she also teach and develop lectures on partial differential equations (analytical and numerical), and Computational modeling of flow and transport. Often times she is invited as a lecturer in workshops and short courses. Together with her students, until now she has actively involved in various research collaborations with colleagues from home institutions or other universities in Indonesia and abroad. 